



\title{
Sólitons a temperatura finita: correções quânticas e térmicas à massa
}

\author{
Luana Perez França
}

Orientador: Prof. Dr. Adilson José da Silva

Dissertação apresentada ao Instituto de Física da Universidade de São Paulo para a obtenção do título de Mestre em Ciências.

Banca Examinadora:

Prof. Dr. Adilson José da Silva (IF-USP)

Prof. Dr. Fernando Tadeu Caldeira Brandt (IF-USP)

Prof. Dr. Enrique Alberto Gallegos Collado (ICT-UNIFAL) 
FICHA CATALOGRÁFICA

Preparada pelo Serviço de Biblioteca e Informação do Instituto de Física da Universidade de São Paulo

França, Luana Perez
Sólitons a temperatura finita: correções quânticas e
térmicas à massa. São Paulo, 2014.
Dissertação (Mestrado) - Universidade de São Paulo.
Instituto de Física. Departamento de Física Matemática
Orientador: Prof. Dr. Adilson José da Silva
Área de Concentração: Física
Unitermos: 1.Física; 2. Teoria de campos e ondas; 3. Física
matemática; 4. Física teórica; 5. Mecânica estatística quântica.
USP/IF/SBI-078/2014




\section{Agradecimentos}

Ao meu orientador, Adilson José da Silva, pela compreensão, motivação e, principalmente, pela disposição e paciência em sanar todas as minhas dúvidas;

Aos meus pais, Sônia Maria Perez França e Israel Pinheiro França, e ao meu irmão, Raul Perez França, por todo o apoio, pois sem eles eu não teria chegado até aqui;

Ao meu marido, Marco Aurélio Savoia, por todo o ânimo e força para persistir;

Aos meu colegas do Instituto de Física, especialmente Fabrício Marques do Carmo, Enrique Alberto Gallegos Collado e Victor Peralta Cano, que me aconselharam e ajudaram sempre que possível;

À Capes pela ajuda financeira. 



\section{RESUMO}

Sólitons são soluções clássicas de equações de campos não lineares, que possuem energia finita e densidade de energia localizada. Eles constituem pacotes de energia que se movem de maneira uniforme e não dispersiva, assemelhando-se a partículas estendidas. Quando se estuda um sistema à temperatura finita é possível tecer um paralelo entre a teoria quântica de campos e a mecânica estatística. Neste trabalho calculamos, na aproximação de um laço, a correção quântica à massa do kink do modelo $\lambda \phi^{4}$ acoplado a um campo fermiônico. As contribuições bosônica e fermiônica são calculadas à temperatura zero e o comportamento das flutuações a temperatura finita também é analisado. 



\begin{abstract}
Solitons are classical solutions of non-linear field equations, that have finite energy and localised energy density. They constitute non-dispersive localised packages of energy moving uniformly, resembling extended particles. When studying a system at finite temperature one can make an analogy between quantum field theory and statistical mechanics. In this work we calculate, in one loop approximation, the quantum correction to the mass of the kink of the model $\lambda \phi^{4}$ coupled to a fermionic field. The bosonic and fermionic contributions are calculated at zero temperature and the behavior of the finite temperature fluctuations are also analysed.
\end{abstract}





\section{Sumário}

$\begin{array}{ll}\text { 1. Introdução } & 1\end{array}$

2. Uma introdução aos sólitons $\quad 7$

2.1. Uma breve visão geral . . . . . . . . . . . . . . . . . . . . . 7

2.2. O modelo $\lambda \phi^{4} \ldots \ldots \ldots \ldots \ldots \ldots$

3. Teoria de Campos a temperatura finita 15

3.1. O formalismo de integrais funcionais . . . . . . . . . . 15

3.2. Representação da função de partição em integrais funcionais . . . . . . 20

3.2.1. Periodicidade . . . . . . . . . . . . . . . 22

3.3. Aproximação de fase estacionária . . . . . . . . . . . . 23

4. Correção quântica da massa do kink no modelo $\lambda \phi^{4} \quad 27$

4.1. Quantização do modelo $\lambda \phi^{4} \ldots \ldots$. . . . . . . . . . . 27

4.2. Cálculo das flutuações bosônicas a temperatura zero . . . . . . . . . 31

4.3. Correção da massa a temperatura zero . . . . . . . . . . . . . 37

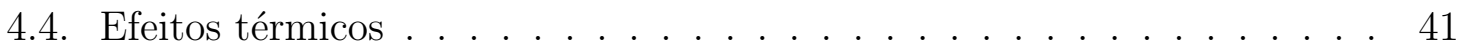

5. Modelo $\lambda \phi^{4}$ acoplado a campo Fermiônico 45

5.1. Férmions a temperatura finita . . . . . . . . . . . 45

5.1.1. Antiperiodicidade . . . . . . . . . . . . 46 
5.2. Quantização do modelo $\lambda \phi^{4}$ acoplado a campo Fermiônico . . . . . . . 47

5.3. Cálculo das flutuações fermiônicas . . . . . . . . . . . . . . . . . . . . . 49

5.4. Correção da massa a temperatura zero . . . . . . . . . . . . . . 55

5.5. Efeitos térmicos . . . . . . . . . . . . . . . . . . 59

6. Conclusões 63

$\begin{array}{ll}\text { Apêndice A. Integrais funcionais } & 65\end{array}$

A.1. Integral funcional gaussiana para variáveis bosônicas . . . . . . . . . . 65

A.2. Integral funcional gaussiana para variáveis fermiônicas . . . . . . . . . 67

Apêndice B. Demonstração da identidade $\ln \operatorname{det} A=\operatorname{tr} \ln A \quad \mathbf{7 1}$

$\begin{array}{ll}\text { Apêndice C. Alguns resultados importantes } & 75\end{array}$

C.1. Cálculo de $\sum_{n=-\infty}^{\infty} \ln \left(\alpha^{2}+\frac{4 \pi^{2} n^{2}}{\beta^{2}}\right) \ldots \ldots \ldots \ldots . \ldots \ldots . \ldots \ldots$

C.2. Cálculo de $\sum_{n=-\infty}^{\infty} \ln \left(\alpha^{2}+\frac{(2 n+1)^{2} \pi^{2}}{\beta^{2}}\right) \ldots \ldots \ldots \ldots . \ldots . \ldots . \ldots 76$

C.3. Modo zero . . . . . . . . . . . . . . . . . . . . . . . . 77

C.4. Integral divergente . . . . . . . . . . . . . . . . 77

$\begin{array}{ll}\text { Apêndice D. Conjugação de carga } & 79\end{array}$

$\begin{array}{lll}\text { Apêndice E. Propagadores de Feynman bosônico e fermiônico } & 81\end{array}$

E.1. Propagador bosônico . . . . . . . . . . . . . . . . . . . . . . . 81

E.2. Propagador fermiônico . . . . . . . . . . . . . . . . . . . 83

$\begin{array}{ll}\text { Apêndice F. Renormalização } & 87\end{array}$

F.1. Campo bosônico . . . . . . . . . . . . . . . . . . . . 87

F.2. Campo bosônico acoplado a campo fermiônico . . . . . . . . . . . . . 95 
Referências Bibliográficas 



\section{Introdução}

Em 1834, J. Scott Russel estava observando o movimento de um barco sendo puxado por dois cavalos no canal de Edimburgo. Quando o barco subitamente freou criou uma grande onda solitária de forma arredondada e bem definida. Ele seguiu a onda formada por um longo trecho e observou que ela se propagava numa velocidade constante e mantinha sua forma original inalterada.

O que Russel observou era um modelo de sólitons. Matematicamente, sólitons são soluções clássicas de equações não lineares de campos, na métrica de Minkowski, que possuem energia finita e densidade de energia localizada. Se propagam com velocidade uniforme e sem mudar a forma. Por constituírem pacotes de energia que se movem de maneira uniforme e não dispersiva se assemelham a partículas estendidas [1].

Para entender melhor estes conceitos vamos considerar a mais simples das equações de onda relativística, dada por:

$$
\partial^{\mu} \partial_{\mu} \phi=\left(\frac{1}{c^{2}} \frac{\partial^{2}}{\partial t^{2}}-\frac{\partial^{2}}{\partial x^{2}}\right) \phi(x, t)=0
$$

onde $\phi(x, t)$ é um campo escalar em $1+1$ dimensões e $c$ é a velocidade da luz. Esta equação é linear e não-dispersiva, desta forma, suas soluções possuem as seguintes características [2]:

1. Qualquer função da forma $f(x \pm c t)$ é uma solução e podemos construir um pacote de onda localizado que se move com velocidade uniforme e sem mudar 
sua forma. Por exemplo:

$$
f(x \pm c t)=\int d k\left[a_{1}(k) \cos (k x \pm \omega t)+a_{2}(k) \sin (k x \pm \omega t)\right] .
$$

O pacote de onda viaja sem mudar sua forma e com velocidade constante $c$ pelo fato de que todas suas componentes são ondas planas com a mesma velocidade $\omega / k=c$.

2. Dadas duas soluções de pacotes de onda, após uma colisão, recuperam sua forma e velocidade originais num tempo assintótico $(t \rightarrow+\infty)$.

No entanto, a maioria das equações de onda são mais complicadas, podendo conter termos não-lineares e/ou dispersivos. A título de comparação, consideremos agora a equação de Klein-Gordon em duas dimensões [3]:

$$
\left(\square+\frac{m^{2} c^{2}}{\hbar^{2}}\right) \phi(x, t) \equiv\left(\frac{1}{c^{2}} \frac{\partial^{2}}{\partial t^{2}}-\frac{\partial^{2}}{\partial x^{2}}+\frac{m^{2} c^{2}}{\hbar^{2}}\right) \phi(x, t)=0 .
$$

Esta equação também é linear e ondas planas $\cos (k x \pm \omega t)$ e $\sin (k x \pm \omega t)$ são soluções. Mas agora $\omega^{2}=k^{2} c^{2}+m^{2} c^{4}$, portanto, diferentes comprimentos de onda viajam a diferentes velocidades, já que $\omega(k) / k=v$, o que torna a equação dispersiva. Portanto, esta equação não segue nenhuma das características (1) e (2).

Consideremos adicionar um termo não-linear à equação 1.1:

$$
\left(\frac{1}{c^{2}} \frac{\partial^{2}}{\partial t^{2}}-\frac{\partial^{2}}{\partial x^{2}}\right) \phi(x, t)+\phi^{3}(x, t)=0
$$

Para este caso em particular, as soluções são dispersivas. Entretanto, é possível para algumas equações, onde termos não-lineares e dispersivos estão presentes, que seus efeitos sejam equilibrados de tal forma a termos soluções que obedecem a característica (1), chamadas ondas solitárias. Em alguns casos, além de obeceder a característica (1), certas soluções também obedecem a característica (2), sendo então chamadas de 
sólitons.

Vamos nos restringir às equações de campo que tenham associada uma densidade de energia $\epsilon(x, t)$ que é função dos campos $\phi_{i}(x, t)$. A integral espacial desta densidade leva à energia total conservada $E\left[\phi_{i}\right]$. Diz-se que temos "soluções localizadas" quando as densidades de energia são localizadas no espaço, ou seja, são finitas dentro de uma região finita e tendem a zero quando as coordenadas espaciais tendem a infinito. Uma onda solitária (a partir de agora chamada sóliton) define-se como a solução localizada de qualquer equação não-linear de campo cuja densidade de energia, além de ser localizada, possui uma dependência no espaço-tempo da forma:

$$
\epsilon(x, t)=\epsilon(x-u t)
$$

ou seja, a densidade de energia se mantém constante se movendo com uma velocidade constante $([2]$ e [4]).

Classicamente, o estado de energia mais baixo de uma partícula sujeita a um potencial de um oscilador harmônico clássico, $m \omega^{2} x^{2} / 2$, possui energia nula e corresponde à partícula em repouso em $x=0$. Na teoria quântica este estado não é permitido. $\mathrm{O}$ princípio de incerteza de Heisenberg não permite que a partícula esteja em repouso (momento definido nulo) para determinado valor de $x$ (posição definida). Quando a partícula se encontra no estado fundamental, a oscilação quântica faz sua energia aumentar de $\hbar \omega / 2$. Os estados excitados ocorrem em energias $(n+1 / 2) \hbar \omega$.

Assim como o oscilador harmônico clássico é modificado por efeitos quânticos, qualquer solução clássica de equações de campo também será modificada. Partículas elementares são descritas por teorias quânticas, enquanto que sólitons são soluções de equações de campo clássicas. Um dos pontos mais importantes da teoria é fazer uma correspondência entre ambos através da versão quantizada dos sólitons. Vários métodos foram desenvolvidos nos anos setenta relativos a quantização de sólitons, mas, 
no geral, constituem uma generalização da expansão semiclássica da teoria quântica não relativística (método WKB) aplicada a teoria de campos relativística [5]. Esta expansão semiclássica parte de uma solução clássica ao redor da qual se quantizam flutuações. Com a quantização podemos associar a uma solução solitônica clássica vários estados de partículas quânticas. Desta forma, podemos obter propriedades dos sólitons como a correção de sua energia (ou massa para caso estático) devido às flutuações quânticas.

Quando se estuda um sistema a temperatura finita, podemos tecer um paralelo entre a teoria quântica de campos e a mecânica estatística. O comportamento estatístico de um sistema quântico, em equilíbrio térmico, é estudado através de um ensemble. Na mecânica estatística podemos encontrar três tipos de ensemble. O ensemble microcanônico é usado para descrever um sistema isolado com uma energia $E$, número de partículas $N$ e volume fixos. O ensemble canônico se utiliza para descrever um sistema em contato com um reservatório de calor a temperatura $T$ e o sistema pode trocar energia com o reservatório. Desta forma, $T, N$ e $V$ são variáveis fixas. No ensemble grande canônico, o sistema pode trocar partículas e energia com o reservatório. Neste caso, $T, V$ e o potencial químico, $\mu$, são variáveis fixas. Ao longo desta dissertação usaremos apenas o ensemble canônico para descrever nosso sistema.

A função de partição da mecânica estatística é o traço da matriz densidade do sistema, que no ensemble canônico é dada por:

$$
Z=\operatorname{Tr} \rho=\operatorname{Tr} e^{-\beta H}
$$

onde $H$ é a hamiltoniana do sistema e $\beta$ representa o inverso da temperatura de equilíbrio (estamos assumindo que a constante de Boltzmann $k=1$, caso contrário, $\beta=1 / k T)$.

O formalismo de Matsubara (tempo imaginário) é ideal para o estudo de sistemas 
estáticos em equilíbrio [6]. Neste formalismo, a função de partição é representada por uma integral de trajetória onde a ação é definida no espaço euclidiano e o tempo é integrado num intervalo finito, em que o limite do tempo é igual ao inverso da temperatura. Além disso, as variáveis de campo desta ação euclidiana devem satisfazer condições periódicas ou antiperiódicas de período $\beta$, conforme estejamos lidando com bósons ou férmions, respectivamente. O formalismo de Matsubara fornece uma maneira de avaliar a função de partição de forma perturbativa usando o método da expansão semiclássica.

O que faremos neste trabalho será estudar um modelo de sóliton ${ }^{1}\left(\lambda \phi^{4}\right)$ em duas dimensões a temperatura finita e determinar a correção à sua energia devido a flutuações quânticas e térmicas. Primeiro analisaremos o modelo puramente bosônico e depois veremos como se comporta quando o acoplamos a um campo fermiônico. Isto foi feito no trabalho [7] em quatro dimensões.

A dissertação está organizada como segue. No capítulo 2, fazemos uma introdução aos sólitons e apresentamos o modelo a ser estudado, $\lambda \phi^{4}$. No capítulo 3 é desenvolvido o formalismo de integrais de trajetória e temperatura finita de tal forma a quantizar o modelo. No capítulo 4 é feita a correção da massa quântica a temperatura zero e o cálculo das flutuações a temperatura finita. Finalmente, no capítulo 5 é feita a correção da massa quântica, a temperatura zero, do modelo $\lambda \phi^{4}$ acoplado a campo fermiônico e o cálculo das flutuações a temperatura finita. Os apêndices contêm alguns detalhes sobre os cálculos e demonstração de fórmulas importantes utilizados no decorrer da dissertação.

\footnotetext{
${ }^{1} \mathrm{Na}$ verdade, este modelo é uma onda solitária, mas na literatura convencionou-se chamá-lo também de sóliton, assim continuaremos utilizando esta definição.
} 



\section{Uma introdução aos sólitons}

Como foi explicado na introdução, do ponto de vista matemático, sólitons são soluções clássicas localizadas de equações não lineares de campo. Em referenciais em repouso são estáticos e em referenciais em movimento constituem pulsos de energia se propagando com velocidade uniforme e sem dissipação, ou seja, a densidade de energia em um certo ponto não se anula quando o tempo tende a infinito. Neste capítulo apresentaremos em detalhe o modelo $\lambda \phi^{4}$. Este modelo teórico é bastante simples e possui um único campo escalar, $\phi$, em $1+1$ dimensões.

\subsection{Uma breve visão geral}

Consideremos um campo escalar $\phi(x, t)$ em duas dimensões governado por uma densidade lagrangiana:

$$
\mathcal{L}=\frac{1}{2}(\dot{\phi})^{2}-\frac{1}{2}\left(\phi^{\prime}\right)^{2}-U(\phi)
$$

onde $(\cdot)$ e $(')$ representam diferenciação com respeito ao tempo e ao espaço (variável $\mathrm{x}$ ), respectivamente. A velocidade da luz é definida como 1. O 'potencial', U( $\phi)$, é qualquer função de $\phi$ que tende a zero para um valor (ou vários) de $\phi$.

Podemos determinar a equação de movimento aplicando a esta Lagrangiana a equação de Euler-Lagrange:

$$
\partial_{\mu} \frac{\partial \mathcal{L}}{\partial\left(\partial_{\mu} \phi\right)}-\frac{\partial \mathcal{L}}{\partial \phi}=0
$$


onde o índice $\mu=0,1$. Obtemos então:

$$
\square \phi \equiv \ddot{\phi}-\phi^{\prime \prime}=-\frac{\partial U}{\partial \phi}(x, t)
$$

Esta é nossa equação de onda, cujos termos não lineares dependem da escolha de $\mathrm{U}(\phi)$. Quando $t$ varia, a energia total, $E$, é conservada e dada por:

$$
E[\phi]=\int_{-\infty}^{\infty} d x\left[\frac{1}{2}(\dot{\phi})^{2}+\frac{1}{2}\left(\phi^{\prime}\right)^{2}+U(\phi)\right]
$$

Suponhamos que o 'potencial' $\mathrm{U}(\phi)$ pode assumir valores mínimos (que também correspondem aos seus zeros) em $M \geq 1$ pontos. Ou seja:

$$
U(\phi)=0 \quad \text { para } \quad \phi=g^{i} ; \quad i=1, \ldots, M .
$$

A energia, $E[\phi]$, também é minimizada quando o campo $\phi(x, t)$ é constante no espaço-tempo e assume qualquer um destes valores. Ou seja:

$$
E[\phi]=0 \quad \text { para } \quad \phi(x, t)=g^{i} ; \quad i=1, \ldots, M
$$

Estamos interessados apenas em soluções estáticas. Neste caso (2.3) se reduz a

$$
\phi^{\prime \prime}(x) \equiv \frac{d^{2} \phi}{d x^{2}}=\frac{d U}{d \phi}(x)
$$

Uma onda solitária (ou sóliton) deve possuir energia finita e uma densidade de energia localizada. Em vista de (2.5) o campo de um sóliton deve se aproximar, quando $x \rightarrow \pm \infty$, a algum dos valores $g^{i}$. Se $U(\phi)$ possuir um único mínimo em $\phi=g$, então nossa solução estática deve ter $\phi \rightarrow g$ quando $x \rightarrow \pm \infty$. Caso existam mínimos degenerados $(M>1)$, então $\phi(x)$ pode tender a qualquer um dos valores de $\mathrm{g}^{i}$ quando $x \rightarrow-\infty$ e, ao mesmo valor, ou mesmo outro valor quando $x \rightarrow+\infty$.

Podemos determinar soluções para as equações de movimento (2.7). Se multiplicar- 
mos ambos os lados por $\phi^{\prime}$ e integrarmos em x, teremos:

$$
\int \phi^{\prime} \phi^{\prime \prime} d x=\int \frac{d U}{d \phi} \phi^{\prime} d x
$$

$\mathrm{Ou}$

$$
\frac{1}{2}\left(\phi^{\prime}\right)^{2}=U(\phi)
$$

Tirando a raiz quadrada de (2.9):

$$
\frac{d \phi}{d x}= \pm[2 U(\phi)]^{\frac{1}{2}}
$$

A equação pode ser integrada mais uma vez, levando a:

$$
x-x_{0}= \pm \int_{\phi\left(x_{0}\right)}^{\phi(x)} \frac{d \bar{\phi}}{[2 U(\bar{\phi})]^{\frac{1}{2}}},
$$

onde a constante de integração $x_{0}$ corresponde a um ponto arbitrário no espaço em que o campo tem valor $\phi\left(x_{0}\right)$. A solução $\phi(x)$ pode ser obtida, em princípio, integrando (2.11) e invertendo o resultado.

\subsection{0 modelo $\lambda \phi^{4}$}

A seguir estudaremos a solução "kink" (e antikink) da teoria $\lambda \phi^{4}$. Neste caso, a densidade lagrangiana tem a forma de (2.1) com

$$
U(\phi)=\frac{\lambda}{4}\left(\phi^{2}-m^{2} / \lambda\right)^{2}
$$

onde $\lambda$ e $\mathrm{m}^{2}$ são constantes positivas.

O potencial $U(\phi)$ possui dois mínimos degenerados (Veja figura 2.1), dados por:

$$
\phi= \pm m / \sqrt{\lambda}
$$




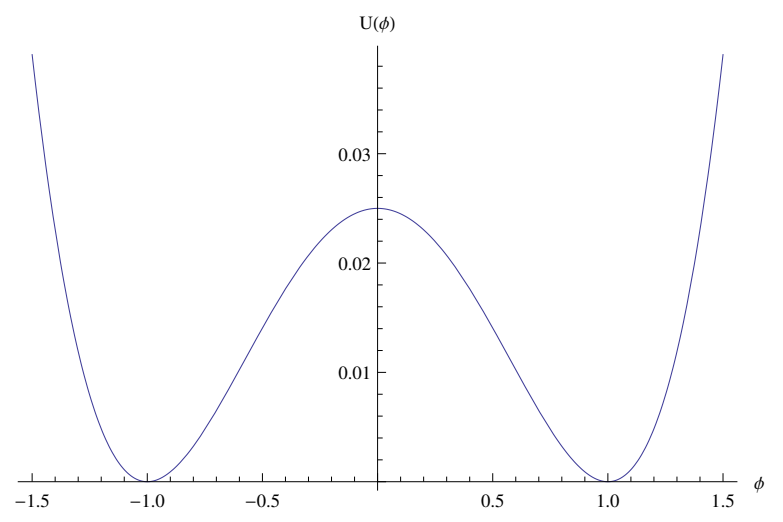

Figure 2.1.: Gráfico do potencial do modelo $\lambda \phi^{4}$ para $\lambda=0,1$ e $m^{2} / \lambda=1$.

A densidade de energia se anula quando $\phi(x, t)$ assume algum destes valores, que representam vácuos da teoria. Soluções solitônicas, caso existam, devem tender a algum destes valores quando $x \rightarrow-\infty$ e a outro valor ou mesmo quando $x \rightarrow+\infty$.

Neste caso, teremos quatro configurações possíveis dadas por:

$$
\begin{aligned}
& \text { Vácuo } \rightarrow \begin{cases}\phi(x)=-m / \sqrt{\lambda} & \text { para } x \rightarrow-\infty ; \\
\phi(x)=-m / \sqrt{\lambda} & \text { para } x \rightarrow+\infty\end{cases} \\
& \text { Vácuo } \rightarrow \begin{cases}\phi(x)=+m / \sqrt{\lambda} & \text { para } x \rightarrow-\infty \\
\phi(x)=+m / \sqrt{\lambda} & \text { para } x \rightarrow+\infty\end{cases} \\
& \text { Setor kink } \rightarrow \begin{cases}\phi(x)=-m / \sqrt{\lambda} & \text { para } x \rightarrow-\infty \\
\phi(x)=+m / \sqrt{\lambda} & \text { para } x \rightarrow+\infty\end{cases} \\
& \text { Setor antikink } \rightarrow \begin{cases}\phi(x)=-m / \sqrt{\lambda} & \text { para } x \rightarrow+\infty \\
\phi(x)=+m / \sqrt{\lambda} & \text { para } x \rightarrow-\infty\end{cases}
\end{aligned}
$$

Nos primeiros dois casos temos os vácuos da teoria. No terceiro setor, a função $\phi(x)$ deve ir de $-m / \sqrt{\lambda}$ a $+m / \sqrt{\lambda}$ quando $x$ vai de $-\infty$ a $+\infty$. Por continuidade do campo, deve haver pelo menos um ponto no espaço, $x_{0}$, tal que $\phi\left(x_{0}\right)=0$. Como $U(0) \neq 0$, a energia potencial na vizinhança de $x_{0}$ não é nula. A solução da equação clássica de movimento que interpola entre estas condições de contorno é o chamado kink da teoria 
$\lambda \phi^{4}$. O quarto setor, conhecido como antikink, representa a configuração contrária, em que a função $\phi(x)$ deve ir de $-m / \sqrt{\lambda}$ a $+m / \sqrt{\lambda}$ quando $x$ vai de $+\infty$ a $-\infty$.

Cada um destes setores está associado a uma carga topológica constante definida da seguinte forma [2]:

$$
Q=(\sqrt{\lambda} / m)[\phi(x=\infty)-\phi(x=-\infty)]
$$

A carga dos setores vácuo é dada por $Q=0$, no caso do kink, $Q=1 \mathrm{e}$, para o antikink, temos $Q=-1$. Setores com cargas topológicas distintas não podem decair uns nos outros devido à energia ser finita. Assim, podemos concluir que as soluções são estáveis. A seguir vamos determinar as soluções da equação de movimento.

A equação de movimento estática (2.7) é dada por:

$$
\phi^{\prime \prime}=d U / d \phi=\lambda \phi^{3}-m^{2} \phi
$$

e pode ser resolvida usando (2.11), neste caso:

$$
x-x_{0}= \pm \int_{\phi(x)}^{\phi\left(x_{0}\right)} \frac{d \bar{\phi}}{\sqrt{\lambda / 2}\left(\bar{\phi}^{2}-m^{2} / \lambda\right)} .
$$

Escolhendo $\phi\left(x_{0}\right)=0$, integrando em $\bar{\phi}$ e invertendo o resultado, obtemos:

$$
\phi_{k(a)}(x)= \pm(m / \sqrt{\lambda}) \tanh \left[m\left(x-x_{0}\right) / \sqrt{2}\right]
$$

O sinal \pm indica que há duas ondas solitárias: o "kink" (com o sinal positivo) e o "antikink". Pode se verificar que esta solução tende aos valores assintóticos $\phi=$ $\pm m / \sqrt{\lambda}$ como deveria. Para melhor entendimento observe o gráfico da solução kink na figura 2.2 a seguir:

A densidade de energia da solução kink (Veja gráfico 2.3), usando (2.10), é dada 


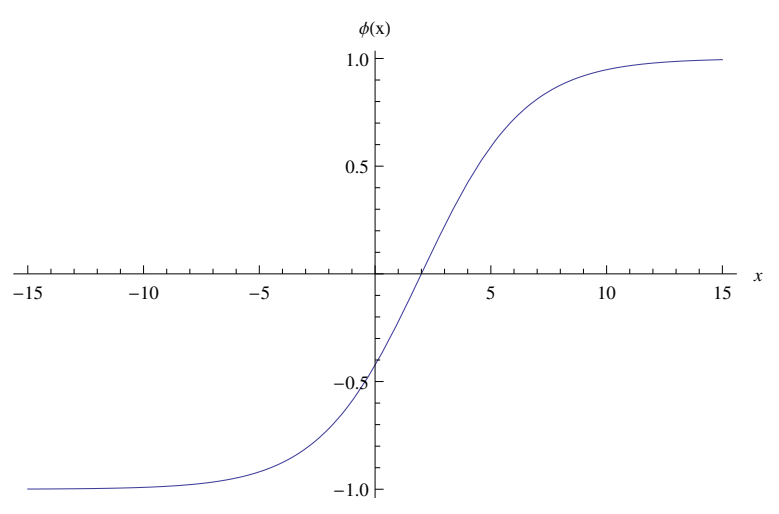

Figure 2.2.: Gráfico da solução kink para $\lambda=0,1$ e $m^{2} / \lambda=1$.

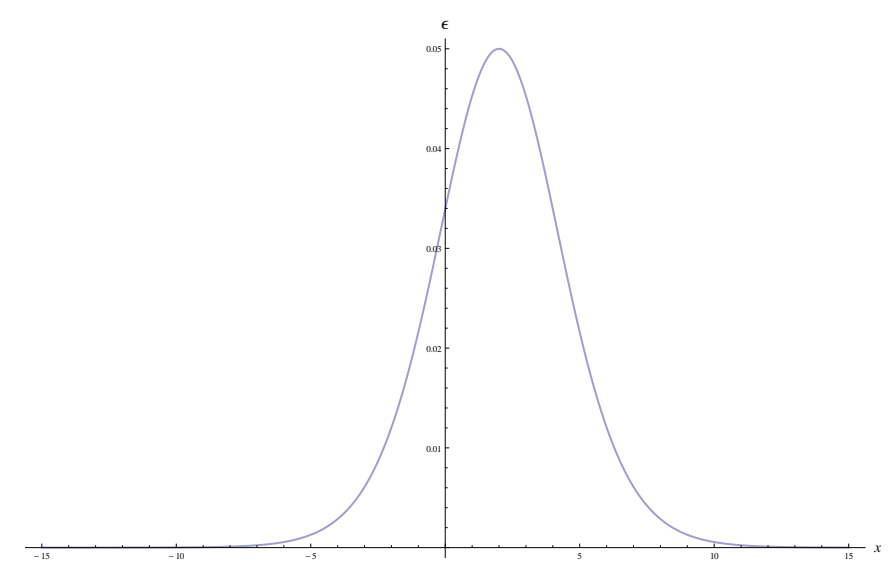

Figure 2.3.: Gráfico da densidade de energia do kink para $\lambda=0,1$ e $m^{2} / \lambda=1$.

por:

$$
\begin{aligned}
& \varepsilon=\frac{1}{2}\left(\phi^{\prime}\right)^{2}+U\left(\phi_{k}\right)=2 U\left(\phi_{k}\right) \\
& \varepsilon=\left(m^{4} / 2 \lambda\right) \operatorname{sech}^{4}\left[m\left(x-x_{0}\right) / \sqrt{2}\right] .
\end{aligned}
$$

A energia total do kink, ou massa para o caso estático, é dada por:

$$
\begin{aligned}
M_{c l} & =\int_{-\infty}^{\infty} d x \varepsilon=\frac{m^{4}}{2 \lambda} \int_{-\infty}^{\infty} d x \operatorname{sech}^{4}\left[m\left(x-x_{0}\right) / \sqrt{2}\right] \\
& =\frac{m^{4}}{2 \lambda} \int_{-\infty}^{\infty} d x \operatorname{sech}^{2}\left[m\left(x-x_{0}\right) / \sqrt{2}\right]\left(1-\tanh ^{2}\left[m\left(x-x_{0}\right) / \sqrt{2}\right]\right) \\
& =\frac{m^{4}}{2 \lambda} \frac{\sqrt{2}}{m}\left[\tanh \left[m\left(x-x_{0}\right) / \sqrt{2}\right]-\frac{1}{3} \tanh ^{3}\left[m\left(x-x_{0}\right) / \sqrt{2}\right]\right]_{-\infty}^{+\infty}=\frac{2 \sqrt{2}}{3} \frac{m^{3}}{\lambda}
\end{aligned}
$$


ou seja:

$$
M_{c l}=\frac{2 \sqrt{2}}{3} \frac{m^{3}}{\lambda}
$$

Este resultado é a massa clássica do sistema, que seria exata se descontados os efeitos quânticos ${ }^{1}$.

Como o sistema é relativístico e invariante de Lorentz, podemos obter a solução dependente do tempo fazendo uma transformação de Lorentz na solução estática do kink 2.21. Obtem-se:

$$
\phi_{u}(x)=\frac{m}{\lambda} \tanh \left[\frac{m}{\sqrt{2}}\left(\frac{\left(x-x_{0}\right)-u t}{\sqrt{1-u^{2}}}\right)\right]
$$

onde $1>u>-1$ é a velocidade. Assim, a energia total do kink é dada por:

$$
E\left[\phi_{u}\right]=\int_{-\infty}^{\infty} d x\left[\frac{1}{2}\left(\dot{\phi}_{u}\right)^{2}+\frac{1}{2}\left(\phi_{u}^{\prime}\right)^{2}+U\left(\phi_{u}\right)\right]=\frac{M_{c l}}{\sqrt{1-u^{2}}}
$$

onde $M_{c l}$ é a energia do kink estático. Observa-se que a relação entre 2.26 e 2.24 é a mesma que a equação massa-energia de Einstein para uma partícula.

\footnotetext{
${ }^{1} \mathrm{~A}$ dimensão da constante $\lambda$ é $\left[M^{2}\right]$ e, portanto, a dimensão de $M_{c l}$ é $\left[M^{1}\right]$ como deveria.
} 



\section{Teoria de Campos a temperatura finita}

Neste capítulo apresentamos o formalismo de integrais funcionais (seção 3.1) em Teoria de Campos, que será utilizado para representar a função de partição da Mecânica Estatística (seção 3.2), tecendo, assim, um paralelo entre ambas as teorias. Isto será feito para o caso puramente bosônico. Na seção 3.3 será mostrado um método de aproximação muito utilizado para determinar o valor da função de partição: a aproximação de fase estacionária.

\subsection{O formalismo de integrais funcionais}

Integrais funcionais em teoria de campos correspondem a generalizações de integrais de trajetória na mecânica quântica não relativística.

Vamos estudar o exemplo simples de um sistema não relativístico com um grau de liberdade, ou seja, uma partícula se movendo livremente ou sob a influência de um potencial em uma dimensão espacial. Segundo a formulação de Hamilton da mecânica clássica, o sistema é descrito por uma coordenada espacial $q$ e a coordenada do momento canonicamente conjugado $p$ [8]. Sob quantização canônica as coordenadas são representadas por operadores que obedecem a seguinte relação de comutação:

$$
[\hat{p}, \hat{q}]=-i \hbar
$$


Os autoestados dos operadores, no formalismo de Schröedinger, são dados por:

$$
\begin{aligned}
& \hat{q}|q\rangle=q|q\rangle \\
& \hat{p}|p\rangle=p|p\rangle
\end{aligned}
$$

e obedecem às seguintes relações de completeza e ortogonalidade:

$$
\begin{aligned}
\left\langle q^{\prime} \mid q\right\rangle & =\delta\left(q^{\prime}-q\right) \\
\int d q|q\rangle\langle q| & =1
\end{aligned}
$$

e

$$
\begin{aligned}
\left\langle p^{\prime} \mid p\right\rangle & =2 \pi \hbar \delta\left(p^{\prime}-p\right) \\
\int \frac{d p}{2 \pi \hbar}|p\rangle\langle p| & =1 .
\end{aligned}
$$

A representação no espaço de coordenadas do autoestado do momento é uma onda plana:

$$
\langle q \mid p\rangle=e^{i p q / \hbar}
$$

O estado quântico do sistema é descrito por um vetor $|\psi(t)\rangle$ no espaço de Hilbert e a função de onda correspondente no espaço de coordenadas é dada por:

$$
\psi(q, t)=\langle q \mid \psi(t)\rangle
$$

que obedece à equação de Schröedinger:

$$
i \hbar \frac{\partial}{\partial t} \psi(q, t)=H \psi(q, t)
$$

A função de onda $\psi(q, 0)$ em $t=0$ evolui para $t=T$ da seguinte maneira:

$$
\psi(q, T)=e^{-i H T / \hbar} \psi(q, 0)
$$

No formalismo de Heisenberg os operadores dependem do tempo e satisfazem a equação de Heisenberg [9]:

$$
\left[\hat{H}, \hat{q_{H}}\right]=-i \hbar \dot{\hat{q}}_{H}
$$


Assim, a evolução temporal de um operador de coordenadas é dada por:

$$
\hat{q}_{H}(t)=e^{i \hat{H} t / \hbar} \hat{q} e^{-i \hat{H} t / \hbar}
$$

Tal operador possui autoestados dados por:

$$
\hat{q}_{H}|q, t\rangle=q|q, t\rangle
$$

que evoluem no tempo de acordo com

$$
|q, t\rangle=e^{i \hat{H} t / \hbar}|q, 0\rangle
$$

Além disso, $|q, t\rangle$ obedecem às mesmas relações de completeza e ortogonalidade que $|q\rangle:$

$$
\begin{aligned}
\left\langle q^{\prime}, t \mid q, t\right\rangle & =\left\langle q^{\prime}\left|e^{-i \hat{H} t / \hbar} e^{i \hat{H} t / \hbar}\right| q\right\rangle=\left\langle q^{\prime} \mid q\right\rangle=\delta\left(q^{\prime}-q\right) \\
\int d q|q, t\rangle\langle q, t| & =\int d q e^{i \hat{H} t / \hbar}|q\rangle\langle q| e^{-i \hat{H} t / \hbar}=e^{i \hat{H} t / \hbar} e^{-i \hat{H} t / \hbar}=1 .
\end{aligned}
$$

A amplitude de probabilidade de uma partícula num estado $|q, t\rangle$ passar para um estado $\left|q^{\prime}, t^{\prime}\right\rangle$, ou sua amplitude de transição, é dada por:

$$
\left\langle q^{\prime}, t^{\prime} \mid q, t\right\rangle=\left\langle q^{\prime}\left|e^{-i \hat{H}\left(t^{\prime}-t\right) / \hbar}\right| q\right\rangle
$$

O formalismo de integrais de trajetória permite calcular a amplitude de transição a partir da Hamiltoniana clássica. A seguir vamos ver como obter este resultado.

Primeiramente vamos substituir o intervalo de tempo $\left[t^{\prime}, t\right]$ por pontos equidistantes $t_{n} \mathrm{com}$

$$
t_{n}-t_{n-1}=\epsilon \text {, onde } t^{\prime}-t=N \epsilon .
$$

Levando em conta a relação de completeza 3.13, podemos reescrever 3.14 da seguinte 
forma:

$$
\left\langle q^{\prime}, t^{\prime} \mid q, t\right\rangle=\int d q_{N-1} \cdots \int d q_{2} \int d q_{1}\left\langle q^{\prime}, t^{\prime} \mid q_{N-1}, t_{N-1}\right\rangle \cdots \times\left\langle q_{2}, t_{2} \mid q_{1}, t_{1}\right\rangle\left\langle q_{1}, t_{1} \mid q, t\right\rangle
$$

Cada um dos elementos da integral pode ser aproximado da seguinte forma:

$$
\begin{aligned}
\left\langle q_{n+1}, t_{n+1} \mid q_{n}, t_{n}\right\rangle & =\left\langle q_{n+1}\left|e^{-i \hat{H}(\hat{p}, \hat{q}) \epsilon / \hbar}\right| q_{n}\right\rangle=\left\langle q_{n+1}\left|1-\frac{i \epsilon}{\hbar} \hat{H}(\hat{p}, \hat{q})\right| q_{n}\right\rangle+O\left(\epsilon^{2}\right) \\
& =\left\langle q_{n+1} \mid q_{n}\right\rangle-\frac{i \epsilon}{\hbar}\left\langle q_{n+1}|\hat{H}(\hat{p}, \hat{q})| q_{n}\right\rangle+O\left(\epsilon^{2}\right),
\end{aligned}
$$

onde $\epsilon<<1$.

Vamos calcular os elementos de matriz da hamiltoniana $H$, temos:

$$
\begin{aligned}
\left\langle q_{n+1}|\hat{H}(\hat{p}, \hat{q})| q_{n}\right\rangle & =\int \frac{d p_{n}}{2 \pi \hbar}\left\langle q_{n+1} \mid p_{n}\right\rangle\left\langle p_{n}|\hat{H}(\hat{p}, \hat{q})| q_{n}\right\rangle=\int \frac{d p_{n}}{2 \pi \hbar}\left\langle q_{n+1} \mid p_{n}\right\rangle\left\langle p_{n} \mid q_{n}\right\rangle H\left(p_{n}, q_{n}\right) \\
& =\int \frac{d p_{n}}{2 \pi \hbar} e^{i p_{n} q_{n+1} / \hbar} e^{-i p_{n} q_{n} / \hbar} H\left(p_{n}, q_{n}\right)=\int \frac{d p_{n}}{2 \pi \hbar} e^{i p_{n}\left(q_{n+1}-q_{n}\right) / \hbar} H\left(p_{n}, q_{n}\right) .
\end{aligned}
$$

Então:

$$
\begin{aligned}
\left\langle q_{n+1}, t_{n+1} \mid q_{n}, t_{n}\right\rangle & =\left\langle q_{n+1} \mid q_{n}\right\rangle-\frac{i \epsilon}{\hbar}\left\langle q_{n+1}|\hat{H}(\hat{p}, \hat{q})| q_{n}\right\rangle+O\left(\epsilon^{2}\right) \\
& =\int \frac{d p_{n}}{2 \pi \hbar} e^{i p_{n}\left(q_{n+1}-q_{n}\right) / \hbar}\left(1-\frac{i \epsilon}{\hbar} H\left(p_{n}, q_{n}\right)\right)+O\left(\epsilon^{2}\right) .
\end{aligned}
$$

Fazendo agora o limite $N \rightarrow \infty$ e $\epsilon \rightarrow 0$, podemos reescrever 3.15 da seguinte forma:

$$
\left\langle q^{\prime}, t^{\prime} \mid q, t\right\rangle=\lim _{N \rightarrow \infty} \int \prod_{n=1}^{N-1} d q_{n} \prod_{n=0}^{N-1} \frac{d p_{n}}{2 \pi \hbar} \exp \left(\frac{i \epsilon}{\hbar} \sum_{n=0}^{N-1} p_{n} \frac{q_{n+1}-q_{n}}{\epsilon}\right) \times \prod_{n=0}^{N-1}\left(1-\frac{i \epsilon}{\hbar} H\left(p_{n}, q_{n}\right)\right) .
$$

O integrando desta equação pode ser reescrito da seguinte forma (levando em conta que $\epsilon<<1)$ :

$$
\left\langle q^{\prime}, t^{\prime} \mid q, t\right\rangle=\lim _{N \rightarrow \infty} \int \prod_{n=1}^{N-1} d q_{n} \prod_{n=0}^{N-1} \frac{d p_{n}}{2 \pi \hbar} \exp \left[\frac{i \epsilon}{\hbar} \sum_{n=0}^{N-1}\left(p_{n} \frac{q_{n+1}-q_{n}}{\epsilon}-H\left(p_{n}, q_{n}\right)\right)\right] .
$$


Podemos reescrever 3.20 utilizando a seguinte notação:

$$
\prod_{n=1}^{N-1} d q_{n} \rightarrow \int \mathcal{D} q \quad \text { e } \quad \prod_{n=0}^{N-1} \frac{d p_{n}}{2 \pi \hbar} \rightarrow \int \mathcal{D} p
$$

Além disso, com os limites $N \rightarrow \infty$ e $\epsilon \rightarrow 0$, podemos transformar os termos da exponencial da seguinte forma:

$$
\frac{q_{n+1}-q_{n}}{\epsilon} \rightarrow \dot{q}\left(t_{n}\right), \quad \epsilon \sum_{n=0}^{N-1} f\left(t_{n}\right) \rightarrow \int_{t}^{t^{\prime}} d \tau f(\tau) .
$$

Assim, a expressão da amplitude de transição fica dada por:

$$
\left\langle q^{\prime}, t^{\prime} \mid q, t\right\rangle=\int \mathcal{D} q \int \mathcal{D} p \exp \left[\frac{i}{\hbar} \int_{t}^{t^{\prime}} d \tau(p \dot{q}-H(p, q))\right],
$$

que possui como condicões de contorno $q(t)=q$ e $q\left(t^{\prime}\right)=q^{\prime}$.

Muito frequentemente, a hamiltoniana do sistema possui uma dependência quadrática no momento, da seguinte forma:

$$
H(p, q)=\frac{1}{2 m} p^{2}+V(q)
$$

Desta forma, o termo contido na exponencial em 3.23 corresponde à própria lagrangiana do sistema, tal que:

$$
L \equiv p \dot{q}-H(p, q)
$$

Neste caso, podemos realizar as integrais nos momentos separadamente [10] e obter uma integral apenas nas coordenadas, que é a forma inicialmente proposta por Feynman, dada por:

$$
\left\langle q^{\prime}, t^{\prime} \mid q, t\right\rangle=\mathcal{N} \int \mathcal{D} q \exp \left[\frac{i}{\hbar} \int_{t}^{t^{\prime}} d \tau L\right]
$$

A integral de trajetória correspondente em teoria de campos, para o caso de um 
campo escalar neutro $\phi(x)$, é dada por:

$$
\begin{aligned}
\left\langle\phi_{f}, t_{f} \mid \phi_{i}, t_{i}\right\rangle & =\left\langle\phi_{f}\left|e^{-i\left(t_{f}-t_{i}\right) \hat{H} / \hbar}\right| \phi_{i}\right\rangle \\
& =\int \mathcal{D} \phi \int \mathcal{D} \pi \exp \left[\frac{i}{\hbar} \int_{t_{i}}^{t_{f}} d t \int d^{3} x\left(\pi \partial_{0} \phi-\mathcal{H}(\pi, \phi)\right)\right] \\
& =\int \mathcal{D} \phi \int \mathcal{D} \pi \exp \left[\frac{i}{\hbar} \int_{t_{i}}^{t_{f}} d t \int d^{3} x \mathcal{L}(\phi, \dot{\phi})\right]
\end{aligned}
$$

onde $\mathcal{L}$ é a densidade lagrangiana do sistema e $\pi=\frac{\partial \mathcal{L}}{\partial\left(\partial_{0} \phi\right)}$ é o momento canonicamente conjugado a $\phi$. As condições de contorno são dadas por $\phi\left(\mathbf{x}, t_{f}\right)=\phi_{f}(\mathbf{x})$ e $\phi\left(\mathbf{x}, t_{i}\right)=$ $\phi_{i}(\mathbf{x})$.

Caso a densidade hamiltoniana $\mathcal{H}$ tenha uma dependência quadrática na "velocidade", como, por exemplo:

$$
\mathcal{H}=\frac{1}{2} \pi^{2}+\frac{1}{2} \phi^{\prime 2}+V(\phi)
$$

então podemos executar a integral dependente de $\pi$ separadamente [10] e 3.27 se torna:

$$
\left\langle\phi_{f}, t_{f} \mid \phi_{i}, t_{i}\right\rangle=\mathcal{N} \int \mathcal{D} \phi \exp \left[\frac{i}{\hbar} \int_{t_{i}}^{t_{f}} d t \int d^{3} x \mathcal{L}(\phi, \dot{\phi})\right]
$$

onde

$$
\mathcal{L}=\frac{1}{2} \partial_{\mu} \phi \partial^{\mu} \phi-V(\phi)
$$

\subsection{Representação da função de partição em integrais funcionais}

Nesta seção vamos ver como funciona a teoria de campos a temperatura finita para o caso de campos puramente bosônicos. A partir de agora consideraremos $\hbar=1$.

A técnica de integrais funcionais pode ser aplicada na descrição de sistemas a temperatura finita, fazendo uma analogia entre a mecânica estatística e teoria de campos. De fato, dado um sistema governado por uma hamiltoniana $H$, o primeiro passo para 
se determinar efeitos de temperatura é o cálculo de sua função de partição [11]:

$$
Z=\operatorname{Tr}\left[e^{-\beta H}\right]=\int d \phi_{a}\left\langle\phi_{a}\left|e^{-\beta H}\right| \phi_{a}\right\rangle
$$

onde

$$
\beta=\frac{1}{k T}
$$

sendo $k$ a constante de Boltzmann, que, a partir de agora consideraremos como $k=1$. O traço significa somar sobre todas as configurações de campo que o sistema pode ter. Observa-se que a função de partição $Z$ é bastante similar à amplitude de transição 3.27:

$$
\begin{aligned}
\left\langle\phi_{f}, t_{f} \mid \phi_{i}, t_{i}\right\rangle & =\left\langle\phi_{f}\left|e^{-i\left(t_{f}-t_{i}\right) \hat{H}}\right| \phi_{i}\right\rangle \\
& =\int \mathcal{D} \phi \int \mathcal{D} \pi \exp \left[i \int_{t_{i}}^{t_{f}} d t \int d^{3} x(\pi \dot{\phi}-\mathcal{H})\right] .
\end{aligned}
$$

De fato, podemos expressar $Z$ como uma integral funcional sobre campos e seus momentos conjugados se fizermos as seguintes mudanças:

1. Façamos $\beta=i\left(t_{f}-t_{i}\right)$. Como a origem do tempo é arbitrária, podemos fazer $t_{i}=0$ e $t_{f}=-i \beta$. Nosso tempo é uma variável imaginária $\tau=i t$, ou seja, apenas fizemos uma rotação de Wick passando para o espaço euclidiano de coordenadas $\left(x_{4}=i x_{0}\right)$.

2. Façamos $\phi_{i}=\phi_{f}=\phi_{a}$, ou seja, as configurações inicial e final do campo são iguais, e, como diferem por um "tempo" $\beta$, temos uma configuração periódica, onde $\phi(\mathbf{x}, t)=\phi(\mathbf{x}, t+\beta)$ é a condição de contorno a ser seguida pela integral funcional.

Tomemos como exemplo a lagrangiana 3.30 somada a um termo de massa: $-m^{2} \phi^{2} / 2$. Temos que a ação correspondente no espaço de Minkowski é dada por:

$$
S_{M}=\int d^{4} x \mathcal{L}_{M}=\int d^{4} x\left[\frac{1}{2}\left(\partial_{\mu} \phi\right)^{2}-\frac{1}{2} m^{2} \phi^{2}-V(\phi)\right]
$$


Após a rotação de Wick $\left(x_{0}=-i x_{4}, x^{i}=x_{E}^{i}\right)$, esta expressão toma a forma [12]:

$$
i \int d^{4} x_{E} \mathcal{L}_{E}=i \int d^{4} x_{E}\left[\frac{1}{2}\left(\partial_{E \mu} \phi\right)^{2}+\frac{1}{2} m^{2} \phi^{2}+V(\phi)\right] .
$$

Seguindo esta mudança e, observando que o "tempo" agora varia de 0 a $\beta$, podemos escrever $Z$ como:

$$
Z=\int \mathcal{D} \pi \int \mathcal{D} \phi \exp \left[-\int_{0}^{\beta} d \tau \int d^{3} x(i \pi \dot{\phi}-\mathcal{H})\right]
$$

Para o caso de uma lagrangiana com dependência quadrática na "velocidade", a função de partição pode ser reescrita como [13]:

$$
Z=N \int \mathcal{D} \phi \exp \left[-\int_{0}^{\beta} d \tau \int d^{3} x \mathcal{L}_{E}\right]=N \int \mathcal{D} \phi e^{-S_{E}(\phi)}
$$

onde $S_{E}$ é a ação euclidiana do sistema.

Expressamos $Z$ como uma integral funcional sobre um campo $\phi$ periódico e cuja exponencial possui variável temporal imaginária. A constante de normalização $N$ é irrelevante, já que a multiplicação de $Z$ por qualquer constante não altera a termodinâmica do sistema [13].

\subsubsection{Periodicidade}

Consideremos uma onda plana bosônica no espaço dos momentos:

$$
\phi(x, \tau)=e^{i\left(\mathbf{k} \cdot \mathbf{x}+\omega_{n} \tau\right)} \phi_{n}(\mathbf{k}) .
$$

Para uma função periódica arbitrária, definida no intervalo $0 \leq \tau \leq \beta$, as frequências discretas $\omega_{n}$ podem assumir os valores $\omega_{n}=2 n \pi / \beta$. Isto segue da definição do traço na função de partição 3.31 .

Podemos ver isto mais detalhadamente examinando as propriedades da função de 
Green térmica definida como [14]:

$$
G(x, y ; \tau, 0)=Z^{-1} \operatorname{Tr}\{\hat{\rho} T[\hat{\phi}(x, \tau) \hat{\phi}(y, 0)]\}
$$

onde $\hat{\rho}=e^{-\beta H}$ e $T$ representa o operador produto ordenado, que para bósons é definido como ${ }^{1}$ :

$$
T\left[\hat{\phi}\left(\tau_{1}\right) \hat{\phi}\left(\tau_{2}\right)\right]=\theta\left(\tau_{1}-\tau_{2}\right) \hat{\phi}\left(\tau_{1}\right) \hat{\phi}\left(\tau_{2}\right)+\theta\left(\tau_{2}-\tau_{1}\right) \hat{\phi}\left(\tau_{2}\right) \hat{\phi}\left(\tau_{1}\right)
$$

Usando o fato de que $T$ comuta com $\hat{\rho}$ e a propriedade cíclica do traço, temos:

$$
\begin{aligned}
G_{B}(x, y ; \tau, 0) & =Z^{-1} \operatorname{Tr}\left[e^{-\beta H} \hat{\phi}(x, \tau) \hat{\phi}(y, 0)\right] \\
& =Z^{-1} \operatorname{Tr}\left[\hat{\phi}(y, 0) e^{-\beta H} \hat{\phi}(x, \tau)\right] \\
& =Z^{-1} \operatorname{Tr}\left[e^{-\beta H} e^{\beta H} \hat{\phi}(y, 0) e^{-\beta H} \hat{\phi}(x, \tau)\right] \\
& =Z^{-1} \operatorname{Tr}\left[e^{-\beta H} \hat{\phi}(y, \beta) \hat{\phi}(x, \tau)\right] \\
& =Z^{-1} \operatorname{Tr}\{\rho T[\hat{\phi}(x, \tau) \hat{\phi}(y, \beta)]\} .
\end{aligned}
$$

Isto implica em $\phi(y, 0)=\phi(y, \beta)$ e, portanto, $\omega_{n}=2 n \pi / \beta$.

\subsection{Aproximação de fase estacionária}

Integrais da forma

$$
I \equiv \int d x e^{-a(x)}
$$

podem ser aproximadas expandindo $a(x)$ em torno de um ponto $x_{0}$ em que $a(x)$ é estacionária, isto é, em que $a^{\prime}\left(x_{0}\right)=0$.

Com isso temos:

$$
a(x) \approx a\left(x_{0}\right)+\frac{1}{2}\left(x-x_{0}\right)^{2} a^{\prime \prime}\left(x_{0}\right)+\ldots
$$

\footnotetext{
${ }^{1} \theta$ representa a função degrau, igual a 1 para um argumento positivo e 0 para um argumento negativo.
} 
de onde segue que:

$$
I \approx e^{-a\left(x_{0}\right)} \int d x e^{-\frac{1}{2}\left(x-x_{0}\right)^{2} a^{\prime \prime}\left(x_{0}\right)} .
$$

Desta forma chegamos a uma integral gaussiana (para $a^{\prime \prime}\left(x_{0}\right)>0$ ) fácil de ser resolvida.

Vamos aplicar este método para avaliar as funções de partição. Consideremos o gerador funcional definido no espaço euclidiano, que corresponde também à função de partição do sistema, dado por [15]:

$$
Z=N \int \mathcal{D} \phi e^{-S_{E}[\phi, J]},
$$

onde

$$
S_{E}[\phi, J]=\int d^{4} x\left[\frac{1}{2}\left(\partial_{\mu} \phi\right)^{2}+\frac{1}{2} m^{2} \phi^{2}+V(\phi)-J \phi\right],
$$

sendo $J$ é uma fonte externa.

Expandindo a ação em torno de um ponto $\phi_{0}$, teremos:

$$
\begin{aligned}
S_{E}[\phi, J]=S_{E}\left[\phi_{0}, J\right]+\left.\int d^{4} x \frac{\delta S_{E}}{\delta \phi}\right|_{\phi_{0}}\left(\phi-\phi_{0}\right) & +\left.\frac{1}{2} \int d^{4} x \int d^{4} y \frac{\delta^{2} S_{E}}{\delta \phi(x) \delta \phi(y)}\right|_{\phi_{0}}\left(\phi-\phi_{0}\right)_{x}\left(\phi-\phi_{0}\right)_{y} \\
& +O\left(\phi-\phi_{0}\right)^{p}
\end{aligned}
$$

onde $p \geq 3$.

Se $S_{E}$ for estacionária em $\phi_{0}$, então $\phi_{0}$ obedece à equação clássica de movimento dada por:

$$
\left.\frac{\delta S_{E}}{\delta \phi}\right|_{\phi_{0}}=-\square \phi_{0}+m^{2} \phi_{0}+V^{\prime}\left(\phi_{0}\right)-J=0 .
$$


Assim:

$$
\begin{aligned}
S_{E}[\phi, J]= & S_{E}\left[\phi_{0}, J\right] \\
+ & \frac{1}{2} \int d^{4} x \int d^{4} y\left(-\square_{y} \delta^{4}(x-y)+m^{2} \delta^{4}(x-y)+\left.\frac{d^{2} V}{d \phi(x) d \phi(y)}\right|_{\phi_{0}} \delta^{4}(x-y)\right) \times \\
& \left(\phi-\phi_{0}\right)_{x}\left(\phi-\phi_{0}\right)_{y}+O\left(\phi-\phi_{0}\right)^{p} \\
= & S_{E}\left[\phi_{0}, J\right]+\frac{1}{2} \int d^{4} x\left(\phi-\phi_{0}\right)_{x}\left(-\square+m^{2}+V^{\prime \prime}\left(\phi_{0}\right)\right)\left(\phi-\phi_{0}\right)_{x}+O\left(\phi-\phi_{0}\right)^{p} .
\end{aligned}
$$

Portanto:

$$
\begin{aligned}
& Z=N \int \mathcal{D} \phi e^{-S_{E}[\phi, J]} \\
& \approx N e^{-S_{E}\left[\phi_{0}, J\right]} \int \mathcal{D} \phi \exp \left[-\frac{1}{2} \int d^{4} x\left(\phi-\phi_{0}\right)\left(-\square+m^{2}+V^{\prime \prime}\left(\phi_{0}\right)\right)\left(\phi-\phi_{0}\right)\right] \times \\
& \exp \left[O\left(\phi-\phi_{0}\right)^{p}\right] .
\end{aligned}
$$

Se desprezarmos os termos com $p \geq 3$, teremos uma integral gaussiana dada por:

$$
Z \approx N e^{-S_{E}\left[\phi_{0}, J\right]} \int \mathcal{D} \phi \exp \left[-\frac{1}{2} \int d^{4} x\left(\phi-\phi_{0}\right)\left(-\square+m^{2}+V^{\prime \prime}\left(\phi_{0}\right)\right)\left(\phi-\phi_{0}\right)\right]
$$

cuja solução é (Veja apêndice A.1):

$$
Z=N e^{-S_{E}\left[\phi_{0}, J\right]}\left\{\operatorname{det}\left[-\square+m^{2}+V^{\prime \prime}\left(\phi_{0}\right)\right]\right\}^{-1 / 2} .
$$

Podemos reescrever esta expressão usando a identidade:

$$
\operatorname{det} \hat{A}=e^{\ln \operatorname{det} \hat{A}}
$$

como

$$
Z=N e^{-S_{E}\left[\phi_{0}, J\right]-\frac{1}{2} \ln \operatorname{det}\left[-\square+m^{2}+V^{\prime \prime}\left(\phi_{0}\right)\right]}
$$

Para entender melhor o que este resultado representa, relembremos que o funcional 
gerador no espaço euclidiano é dado por [16]:

$$
Z(J)=e^{-W(J)}
$$

onde $W(J)$ corresponde ao funcional gerador das funções de Green conexas. Tendo isto em mente, se observarmos a equação 3.54, veremos que estamos determinando correções a $W(J)$, ou seja:

$$
W(J)=S_{E}\left[\phi_{0}, J\right]+\frac{1}{2} \ln \operatorname{det}\left[-\square+m^{2}+V^{\prime \prime}\left(\phi_{0}\right)\right]
$$

O primeiro termo é o resultado clássico de ordem $\hbar^{0}$, o segundo termo corresponde à primeira correção quântica de ordem $\hbar$. Esta aproximação é chamada de expansão semiclássica e será usada no decorrer de toda a dissertação. 


\section{Correção quântica da massa do kink no modelo $\lambda \phi^{4}$}

Neste capítulo fazemos o cálculo das flutuações bosônicas do modelo $\lambda \phi^{4}$. Na seção 4.1 apresentamos a função de partição do modelo para o caso do vácuo e do kink e mostramos como determinar seu valor utilizando o método da aproximação de fase estacionária apresentado no capítulo anterior. Na seção 4.2 é feito o cálculo das flutuações bosônicas a temperatura zero. Na seção 4.3, através da técnica de renormalização, obtemos um resultado finito para as flutuações. Finalmente, na seção 4.4 são determinadas as flutuações a temperatura finita.

\subsection{Quantização do modelo $\lambda \phi^{4}$}

A partir de agora vamos atuar no espaço euclidiano em $1+1$ dimensões. O primeiro a ser feito é transformar a lagrangiana do nosso modelo $\lambda \phi^{4}$ para o espaço euclidiano. Para isso devemos aplicar as seguintes relações:

$$
x_{0}=-i x_{4} ; \quad \vec{x}=\overrightarrow{x_{E}}
$$

A densidade lagrangiana no espaço de Minkowski é dada por 2.1:

$$
\mathcal{L}_{M}=\frac{1}{2}(\dot{\phi})^{2}-\frac{1}{2}\left(\phi^{\prime}\right)^{2}-U(\phi)
$$

onde $U(\phi)=\frac{\lambda}{4}\left(\phi^{2}-m^{2} / \lambda\right)^{2}$. 
Assim, no espaço euclidiano, teremos:

$$
\mathcal{L}=\frac{1}{2}(\dot{\phi})^{2}+\frac{1}{2}\left(\phi^{\prime}\right)^{2}+U(\phi)
$$

onde, além de aplicar as transformações 4.1, multiplicamos convenientemente por $(-1)$ (Veja 3.34 e 3.35).

A ação euclidiana é dada por:

$$
S[\phi(x, \tau)]=\int_{0}^{\beta} d \tau \int d \mathbf{x} \mathcal{L}(\phi(x, \tau))
$$

Conforme foi visto na seção 3.2, a função de partição para o caso de um campo escalar $\phi(x, \tau)$ é dada por 3.37:

$$
Z=\operatorname{Tr}\left[e^{-\beta \hat{H}}\right]=N \int \mathcal{D} \phi \exp \left[-\int_{0}^{\beta} d \tau \int d x \mathcal{L}\right]=N \int \mathcal{D} \phi e^{-S[\phi(x, \tau)]}
$$

Precisamos, primeiramente, determinar as condições de contorno no tempo e no espaço de $\phi(x, \tau)$. No caso do tempo, como se trata de um campo escalar, este possui periodicidade, tal que, $\phi(x, 0)=\phi(x, \beta)$ (Veja seção 3.2.1).

Para o caso das condições de contorno no espaço, devemos relembrar que o sistema $\lambda \phi^{4}$ possui quatro setores possíveis de estados quânticos (Veja 2.16 e 2.17):

$$
\phi(\infty, \tau)= \pm m / \sqrt{\lambda} \quad \text { e } \quad \phi(-\infty, \tau)= \pm m / \sqrt{\lambda}
$$

Desta forma, podemos determinar a função de partição sobre cada setor separadamente. Ou seja, na correspondente integral funcional, o campo $\phi(x, \tau)$ deve obedecer às condições de contorno daquele setor. Se estamos interessados no setor do kink, os campos devem obedecer a:

$$
\phi(\infty, \tau)=-\phi(-\infty, \tau)=m / \sqrt{\lambda}
$$


para todo $\tau$.

O objetivo é determinar a função de partição 4.5 através de uma aproximação semiclássica. Conforme foi visto anteriormente, a aproximação semiclássica de $Z\left(\phi_{c}\right)$ é obtida expandindo a ação até termos quadráticos no expoente da integral funcional.

Suponhamos, então, uma solução estável e estática do sóliton $\phi(x, \tau)=\phi_{c}(x)$. Vamos expandir a ação $S$ em torno de $\phi_{c}(x)$ :

$$
\begin{aligned}
S[\phi(x, \tau)]=S\left[\phi_{c}(x)\right]+\left.\int d^{2} x \frac{\delta S}{\delta \phi}\right|_{\phi_{c}}\left(\phi-\phi_{c}\right) & +\left.\frac{1}{2} \int d^{2} x \int d^{2} y \frac{\delta^{2} S}{\delta \phi_{x} \delta \phi_{y}}\right|_{\phi_{c}}\left(\phi-\phi_{c}\right)_{x}\left(\phi-\phi_{c}\right)_{y} \\
& +O\left(\phi-\phi_{c}\right)^{3} .
\end{aligned}
$$

Como $\phi_{c}(x)$ é uma solução clássica, é, portanto, um extremo da ação:

$$
\left.\frac{\delta S}{\delta \phi}\right|_{\phi_{c}}=0
$$

Desprezando os termos da ordem de $\left(\phi-\phi_{c}\right)^{3}$, temos:

$$
\begin{aligned}
S[\phi(x, \tau)] & =S\left[\phi_{c}(x)\right]+\left.\frac{1}{2} \int d^{2} x \int d^{2} y \frac{\delta^{2} S}{\delta \phi_{x} \delta \phi_{y}}\right|_{\phi_{c}}\left(\phi-\phi_{c}\right)_{x}\left(\phi-\phi_{c}\right)_{y} \\
& =S\left[\phi_{c}(x)\right]+\frac{1}{2} \int d^{2} x \Phi\left[-\square+3 \lambda \phi_{c}^{2}-m^{2}\right] \Phi \\
& =S\left[\phi_{c}(x)\right]+\frac{1}{2} \int d^{2} x \Phi \hat{\Omega}_{B}\left(\phi_{c}\right) \Phi
\end{aligned}
$$

onde $\Phi=\left(\phi-\phi_{c}\right)$ e $\hat{\Omega}_{B}\left(\phi_{c}\right)$ é o operador bosônico:

$$
\hat{\Omega}_{B}\left(\phi_{c}\right)=-\square+3 \lambda \phi_{c}^{2}-m^{2}
$$

O resultado clássico é dado por $S\left[\phi_{c}(x)\right]$ :

$$
S\left[\phi_{c}(x)\right]=\int d x \int_{0}^{\beta} d \tau\left\{\frac{1}{2}\left(\partial_{\mu} \phi_{c}\right)^{2}+\frac{\lambda}{4}\left(\phi_{c}^{2}-\frac{m^{2}}{\lambda}\right)^{2}\right\}
$$

Inserindo em 4.5 o resultado obtido em 4.7 , teremos para a função de partição: 


$$
\begin{aligned}
Z\left(\phi_{c}\right) & =\exp \left\{-S\left[\phi_{c}(x)\right]\right\} \int \mathcal{D} \Phi e^{-S_{B}(\Phi)} \\
& =\exp \left\{-S\left[\phi_{c}(x)\right]\right\} \int \mathcal{D} \Phi \exp \left\{-\frac{1}{2} \int d^{2} x\left[\Phi \hat{\Omega}_{B}\left(\phi_{c}\right) \Phi\right]\right\} .
\end{aligned}
$$

Lembrando que o "tempo" se extende de 0 a $\beta=1 / T$ (Veja seção 3.2), sendo $T$ a temperatura do sistema.

Vimos na seção 2.2 que o modelo estudado, $\lambda \phi^{4}$, para ser mais exato o "kink" do sistema, corresponde a uma solução clássica dada por 2.21:

$$
\phi_{k}(x)=(m / \sqrt{\lambda}) \tanh \left[m\left(x-x_{0}\right) / \sqrt{2}\right]
$$

além de seus valores assintóticos dados por:

$$
\phi_{k}(\infty)=-\phi_{k}(-\infty)=m / \sqrt{\lambda}
$$

que representam os vácuos da teoria.

O que importa fisicamente é a diferença entre as energias do kink e no vácuo. Em termos da função de partição correspondente $Z\left(\phi_{c}\right)$, temos para a massa corrigida:

$$
M_{c}=-\frac{1}{\beta} \ln \frac{Z\left(\phi_{k}\right)}{Z\left(\phi_{v}\right)} .
$$

onde $^{1}$

$$
\begin{aligned}
Z\left(\phi_{k}\right)= & \exp \left\{-S\left[\phi_{k}(x)\right]\right\} \times \int \mathcal{D} \Phi \exp \left\{-\frac{1}{2} \int d^{2} x\left[\Phi \hat{\Omega}_{B}\left(\phi_{k}\right) \Phi\right]\right\} \\
=\exp & \left\{-\int d^{2} x\left[\frac{1}{2}\left(\partial_{\mu} \phi_{c}\right)^{2}+\frac{\lambda}{4}\left(\phi_{c}^{2}-\frac{m^{2}}{\lambda}\right)^{2}\right]\right\} \\
& \times \int \mathcal{D} \Phi \exp \left\{-\frac{1}{2} \int d^{2} x\left[\Phi \hat{\Omega}_{B}\left(\phi_{k}\right) \Phi\right]\right\} \\
= & \exp \left[-\beta M_{c l}\right] \times\left\{C\left[\operatorname{det} \hat{\Omega}_{B}\left(\phi_{k}\right)\right]^{-1 / 2}\right\}
\end{aligned}
$$

\footnotetext{
${ }^{1} \mathrm{O}$ cálculo das integrais funcionais pode ser visto no apêndice A.1.
} 
em que $M_{c l}=2 \sqrt{2} m^{3} / 3 \lambda$ é a massa clássica, já calculada na seção 2.2 , e

$$
\begin{aligned}
Z\left(\phi_{v}\right) & =\exp \left\{-S\left[\phi_{v}(x)\right]\right\} \times \int \mathcal{D} \Phi \exp \left\{-\frac{1}{2} \int d^{2} x\left[\Phi \hat{\Omega}_{B}\left(\phi_{v}\right) \Phi\right]\right\} \\
& =C\left[\operatorname{det} \hat{\Omega}_{B}\left(\phi_{v}\right)\right]^{-1 / 2},
\end{aligned}
$$

onde $S\left[\phi_{v}(x)\right]=0$.

Então:

$$
\begin{aligned}
M_{c} & =-\frac{1}{\beta} \ln \frac{Z\left(\phi_{k}\right)}{Z\left(\phi_{v}\right)}=-\frac{1}{\beta}\left\{\ln Z\left(\phi_{k}\right)-\ln Z\left(\phi_{v}\right)\right\} \\
& =-\frac{1}{\beta}\left\{-\beta M_{c l}+\ln C-\frac{1}{2} \ln \operatorname{det} \hat{\Omega}_{B}\left(\phi_{k}\right)-\ln C+\frac{1}{2} \ln \operatorname{det} \hat{\Omega}_{B}\left(\phi_{v}\right)\right\} \\
& =M_{c l}+\frac{1}{2 \beta}\left\{\ln \left[\operatorname{det} \hat{\Omega}_{B}\left(\phi_{k}\right)\right]-\ln \left[\operatorname{det} \hat{\Omega}_{B}\left(\phi_{v}\right)\right]\right\} \\
& =M_{c l}+\Gamma_{B}
\end{aligned}
$$

onde $\Gamma_{B}$ é a primeira correção quântica.

Usando a identidade $\ln \operatorname{det} \hat{A}=\operatorname{tr} \ln \hat{A}$ (Veja apêndice B), teremos:

$$
\Gamma_{B}=\frac{1}{2 \beta}\left\{\operatorname{tr} \ln \hat{\Omega}_{B}\left(\phi_{k}\right)-\operatorname{tr} \ln \hat{\Omega}_{B}\left(\phi_{v}\right)\right\} .
$$

Os autovalores deste operador $\hat{\Omega}_{B}\left(\phi_{c}\right)$ representam as flutuações bosônicas em torno da solução clássica $\phi_{c}(x)$ que pode ser o kink ou o vácuo. O que importa é a diferença entre as energias.

\subsection{Cálculo das flutuações bosônicas a temperatura zero}

Nesta seção vamos determinar o valor de 4.17, isto é, a contribuição das flutuações bosônicas à correção da massa.

O operador bosônico é dado por 4.8:

$$
\hat{\Omega}_{B}\left(\phi_{c}\right)=-\square+3 \lambda \phi_{c}^{2}-m^{2} .
$$


Devemos calcular $\ln \operatorname{det} \hat{\Omega}_{B}\left(\phi_{c}\right)$ e sabemos que o determinante de um operador é igual ao produto de seus autovalores. No caso do vácuo do modelo $\lambda \phi^{4}$ a solução clássica é dada por:

$$
\phi_{v}= \pm m / \sqrt{\lambda}
$$

Vamos considerar o resultado $\phi_{v}=+m / \sqrt{\lambda}$, assim:

$$
\hat{\Omega}_{B}\left(\phi_{v}\right)=-\square+2 m^{2} .
$$

No vácuo, as autofunções do operador bosônico correspondem a ondas planas, dadas $\operatorname{por}^{2}$ :

$$
\phi(\mathbf{x}, \tau)=e^{i\left(\mathbf{k} \cdot \mathbf{x}+\omega_{n} \tau\right)} \phi_{n}(\mathbf{k})
$$

Vamos considerar que as ondas estão contidas numa caixa de comprimento $L$, assim:

$$
\begin{gathered}
\phi(-L / 2)=\phi(L / 2) \\
\Rightarrow e^{-i k \frac{L}{2}}=e^{i k \frac{L}{2}}
\end{gathered}
$$

então $k_{r} L=2 \pi r(r=0, \pm 1, \pm 2, \ldots)$.

Para o caso da parte temporal, como estamos lidando com ondas periódicas, temos:

$$
\begin{array}{r}
\phi(\tau)=\phi(\tau+\beta) \\
\Rightarrow e^{i \omega \tau} e^{i 2 \pi n}=e^{i \omega(\tau+\beta)},
\end{array}
$$

então: $\omega_{n} \beta=2 n \pi(n=0, \pm 1, \pm 2, \ldots)$.

Portanto, os autovalores do operador bosônico no vácuo são dados por:

$$
\begin{aligned}
\hat{\Omega}_{B} \phi(x, \tau) & =\varepsilon_{B}\left(\phi_{v}\right) \phi(x, \tau) \\
\Rightarrow \varepsilon_{B}\left(\phi_{v}\right) & =\omega_{n}^{2}+k_{r}^{2}+2 m^{2} .
\end{aligned}
$$

Este resultado corresponde aos quanta da teoria, são flutuações que existem ao redor do vácuo (excitações do vácuo) e são comumente conhecidos como "mésons". O valor

\footnotetext{
${ }^{2}$ Para o caso de uma solução particular.
} 
$2 m^{2}$ corresponde ao quadrado da massa da flutuação.

Para o caso do kink, a solução estática é dada por 2.21:

$$
\phi_{k}(x)=(m / \sqrt{\lambda}) \tanh [m x / \sqrt{2}]
$$

Substituindo na equação 4.18, tal como foi feito para o vácuo, temos:

$$
\hat{\Omega}_{B}\left(\phi_{k}\right)=-\square+2 m^{2}-3 m^{2} \operatorname{sech}^{2}\left(\frac{m x}{\sqrt{2}}\right) .
$$

Então,

$$
\begin{aligned}
\hat{\Omega}_{B}\left(\phi_{k}\right) \phi(x, \tau) & =\varepsilon_{B}\left(\phi_{k}\right) \phi(x, \tau) \\
\Rightarrow\left[-\square+2 m^{2}-3 m^{2} \operatorname{sech}^{2}\left(\frac{m x}{\sqrt{2}}\right)\right] \phi(x, \tau) & =\varepsilon_{B}\left(\phi_{k}\right) \phi(x, \tau),
\end{aligned}
$$

onde $\varepsilon_{B}$ são os autovalores do operador $\hat{\Omega}_{B}\left(\phi_{k}\right)$. Se tentarmos uma solução da forma:

$$
\phi(x, \tau)=e^{i \omega_{n} \tau} \phi_{n}(x),
$$

temos que a equação puramente espacial é dada por:

$$
\left[-\partial_{x}^{2}+2 m^{2}-3 m^{2} \operatorname{sech}^{2}\left(\frac{m x}{\sqrt{2}}\right)\right] \phi(x, \tau)=\left(\varepsilon_{B}-\omega_{n}^{2}\right) \phi(x, \tau) .
$$

Esta equação de autovalores é semelhante à equação de Schröedinger com o potencial de Poschl-Teller, resolvido em detalhe em [17]. Os autovalores são dados por dois níveis discretos e um contínuo:

$$
\varepsilon_{B}\left(\phi_{k}\right)=\left\{\begin{array}{l}
\omega_{n}^{2} \\
\omega_{n}^{2}+\frac{3}{2} m^{2} \\
\omega_{n}^{2}+k^{\prime 2}+2 m^{2}
\end{array}\right.
$$

onde $2 m^{2}$ corresponde ao quadrado da massa da flutuação bosônica e $\omega_{n} \beta=2 n \pi$ $(n=0, \pm 1, \pm 2, \ldots)$, tal como no vácuo.

Observemos na primeira linha de 4.28 que para $n=0$, teremos o chamado "modo 
zero" da teoria, $\omega_{0}=0$. Este valor será excluido dos cálculos, pois não contribui para a massa do sóliton. A presença deste "modo zero" se refere à invariância translacional do kink (para mais detalhes veja [2] e [5]).

O valor de $k^{\prime}$ é determinado a partir das condições de contorno na presença do kink. Colocando as ondas dentro de uma caixa de comprimento $L$ e levando em conta o deslocamento de fase $\delta$ dos estados espalhados, teremos:

$$
\begin{aligned}
& \phi(-L / 2)=\phi(L / 2) \\
& \Rightarrow e^{-i k^{\prime} \frac{L}{2}}=e^{i k^{\prime} \frac{L}{2}} e^{i \delta\left(k^{\prime}\right)},
\end{aligned}
$$

então $k_{r}^{\prime} L+\delta\left(k_{r}^{\prime}\right)=2 r \pi(r=0, \pm 1, \pm 2, \ldots)$.

O valor de $\delta$ é visto em detalhe em [17], [2] e [5] e dado por:

$$
\delta\left(k^{\prime}\right)=2\left\{\pi-\arctan \left[\frac{\sqrt{2} k^{\prime}}{m}\right]-\arctan \left[\frac{\sqrt{2} k^{\prime}}{2 m}\right]\right\} .
$$

Determinadas as flutuações bosônicas na presença do kink e no vácuo, podemos prosseguir ao cálculo de $\Gamma_{B}$.

As condições de contorno no espaço para as flutuações em torno do vácuo são dadas por:

$$
\begin{aligned}
k_{r} L & =2 \pi r \\
\Rightarrow \Delta k & =\frac{2 \pi}{L} .
\end{aligned}
$$

Fazendo $L \rightarrow \infty$, tem-se:

$$
\sum_{r=-\infty}^{\infty} f\left(k_{r}\right)=\sum_{r} f\left(k_{r}\right) \frac{L}{2 \pi} \Delta k \rightarrow \frac{L}{2 \pi} \int_{-\infty}^{+\infty} f(k) d k
$$


Para o caso do kink, temos:

$$
\begin{aligned}
k_{r} L+\delta\left(k_{r}\right) & =2 \pi r \\
\Rightarrow \Delta k L+\frac{d \delta}{d k} \Delta k & =2 \pi \\
\Rightarrow \Delta k\left(1+\frac{1}{L} \frac{d \delta}{d k}\right) & =\frac{2 \pi}{L} .
\end{aligned}
$$

Fazendo $L \rightarrow \infty$, tem-se:

$$
\begin{aligned}
\sum_{r=-\infty}^{\infty} g\left(k_{r}\right) & =\sum_{r} g\left(k_{r}\right) \frac{L}{2 \pi} \Delta k\left(1+\frac{1}{L} \frac{d \delta}{d k}\right) \\
& \Rightarrow \frac{L}{2 \pi} \int_{-\infty}^{+\infty} g(k)\left(1+\frac{1}{L} \frac{d \delta}{d k}\right) d k
\end{aligned}
$$

Usando os autovalores do operador bosônico no vácuo e no kink, equações 4.23 e 4.28 , respectivamente, teremos para 4.17 :

$$
\begin{aligned}
\Gamma_{B}= & \frac{1}{2 \beta}\left\{t r \ln \hat{\Omega}_{B}\left(\phi_{k}\right)-t r \ln \hat{\Omega}_{B}\left(\phi_{v}\right)\right\} \\
= & \frac{1}{2 \beta} \sum_{n=-\infty}^{+\infty}\left\{\ln ^{\prime}\left(\omega_{n}^{2}\right)+\ln \left(\omega_{n}^{2}+\frac{3}{2} m^{2}\right)\right. \\
& \left.+\int_{-\infty}^{+\infty} d k \frac{L}{2 \pi}\left(1+\frac{1}{L} \frac{d \delta}{d k}\right) \ln \left(\omega_{n}^{2}+k^{2}+2 m^{2}\right)-\int_{-\infty}^{+\infty} d k \frac{L}{2 \pi} \ln \left(\omega_{n}^{2}+k^{2}+2 m^{2}\right)\right\} \\
= & \frac{1}{2 \beta} \sum_{n=-\infty}^{+\infty}\left\{\ln ^{\prime}\left(\omega_{n}^{2}\right)+\ln \left(\omega_{n}^{2}+\frac{3}{2} m^{2}\right)+\int_{-\infty}^{+\infty} \frac{d k}{2 \pi} \frac{d \delta}{d k} \ln \left(\omega_{n}^{2}+k^{2}+2 m^{2}\right)\right\}
\end{aligned}
$$

O sinal em $\ln ^{\prime}\left(\omega_{n}^{2}\right)$ significa que o "modo zero" não está sendo levado em conta.

Com $\delta(k)$ dado por 4.30 temos:

$$
\frac{d \delta}{d k}=\frac{-m \sqrt{2}}{m^{2}+2 k^{2}}-\frac{m \sqrt{2}}{2 m^{2}+k^{2}}=-m \sqrt{2}\left(\frac{1}{2 k^{2}+m^{2}}+\frac{1}{k^{2}+2 m^{2}}\right) .
$$


E portanto:

$$
\begin{aligned}
\Gamma_{B}=\frac{1}{2 \beta} \sum_{n=-\infty}^{+\infty}\{ & \ln ^{\prime}\left(\omega_{n}^{2}\right)+\ln \left(\omega_{n}^{2}+\frac{3}{2} m^{2}\right) \\
& \left.\quad-m \sqrt{2} \int_{-\infty}^{+\infty} \frac{d k}{2 \pi}\left(\frac{1}{2 k^{2}+m^{2}}+\frac{1}{k^{2}+2 m^{2}}\right) \ln \left(\omega_{n}^{2}+k^{2}+2 m^{2}\right)\right\} .
\end{aligned}
$$

Usando na expressão acima, a identidade (Veja apêndice C.1):

$$
\sum_{n=-\infty}^{+\infty} \ln \left(\alpha^{2}+\omega_{n}^{2}\right)=\beta \alpha+2 \ln \left(1-e^{-\beta \alpha}\right)+\mathcal{C}
$$

e desprezando o modo zero (Veja apêndice C.3), teremos:

$$
\begin{aligned}
\Gamma_{B} & =\frac{1}{2 \beta}\left\{-2 \ln 2+\beta \sqrt{\frac{3}{2} m^{2}}+2 \ln \left(1-e^{-\beta \sqrt{\frac{3}{2} m^{2}}}\right)\right. \\
& \left.-m 2 \sqrt{2} \int_{-\infty}^{+\infty} \frac{d k}{2 \pi}\left(\frac{1}{2 k^{2}+m^{2}}+\frac{1}{k^{2}+2 m^{2}}\right)\left[\beta \sqrt{k^{2}+2 m^{2}}+2 \ln \left(1-e^{-\beta \sqrt{k^{2}+2 m^{2}}}\right)\right]\right\} \\
\Rightarrow \Gamma_{B} & =-\frac{1}{\beta} \ln 2+\frac{1}{2} \sqrt{\frac{3}{2} m^{2}}+\frac{1}{\beta} \ln \left(1-e^{-\beta \sqrt{\frac{3}{2} m^{2}}}\right) \\
& -m \sqrt{2} \int \frac{d k}{2 \pi}\left(\frac{1}{2 k^{2}+m^{2}}+\frac{1}{k^{2}+2 m^{2}}\right)\left[\sqrt{k^{2}+2 m^{2}}+\frac{2}{\beta} \ln \left(1-e^{-\beta \sqrt{k^{2}+2 m^{2}}}\right)\right] .
\end{aligned}
$$

Esta expressão pode ser separada em uma parte dependente e outra independente da temperatura:

$$
\Gamma_{B}=\Gamma_{B}(0)+\Gamma_{B}(T)
$$

onde $^{3}$

$$
\Gamma_{B}(0)=\frac{1}{2} \sqrt{\frac{3}{2} m^{2}}-m \sqrt{2}(\underbrace{\int \frac{d k}{4 \pi} \frac{\sqrt{k^{2}+2 m^{2}}}{k^{2}+m^{2} / 2}}_{I_{1}}+\underbrace{\int \frac{d k}{2 \pi} \frac{1}{\sqrt{k^{2}+2 m^{2}}}}_{I_{2}}) .
$$

Como podemos observar, as integrais $I_{1}$ e $I_{2}$ são divergentes. A seguir vamos calculá${ }^{3} \mathrm{O}$ termo $k^{2}$ corresponde apenas ao quadrado do módulo do momento $k^{2}=\left|\vec{k}^{2}\right|$. 
las por meio de cut-off.

Temos que $I_{1}$ é uma integral do tipo:

$$
\int_{-\infty}^{\infty} d k \frac{\sqrt{k^{2}+b}}{k^{2}+a}
$$

resolvida em detalhes no apêndice C.4. O resultado é dado por:

$$
I_{1}=\frac{1}{2 \pi}\left[\sqrt{3} \arctan (\sqrt{3})+\ln \Lambda+\ln 2-\ln \sqrt{2 m^{2}}\right]
$$

onde $\Lambda \rightarrow \infty$.

Para $I_{2}$ temos:

$$
I_{2}=\int_{-\Lambda}^{\Lambda} \frac{d k}{2 \pi} \frac{1}{\sqrt{k^{2}+2 m^{2}}}=\int_{0}^{\Lambda} \frac{d k}{\pi} \frac{1}{\sqrt{k^{2}+2 m^{2}}}=\frac{1}{\pi}\left[\ln \Lambda+\ln 2-\ln \sqrt{2 m^{2}}\right] .
$$

Assim:

$$
\Gamma_{B}(0)=\frac{m}{2} \sqrt{\frac{3}{2}}-\frac{m \sqrt{2}}{2 \pi}\left(\sqrt{3} \arctan (\sqrt{3})+3 \ln \Lambda+3 \ln 2-3 \ln \sqrt{2 m^{2}}\right) .
$$

Veremos na próxima seção como renormalizar a divergência contida no logaritmo $\ln \Lambda$.

\subsection{Correção da massa a temperatura zero}

A densidade lagrangiana do modelo $\lambda \phi^{4}$ no espaço euclidiano é dada por:

$$
\mathcal{L}=\frac{1}{2}\left(\partial_{\mu} \phi\right)^{2}+\frac{\lambda}{4}\left(\phi^{2}-\frac{m^{2}}{\lambda}\right)^{2}
$$

A lagrangiana renormalizada é dada por:

$$
\mathcal{L}_{R}=\frac{1}{2}(1+A)\left(\partial_{\mu} \phi\right)^{2}+\frac{1}{2}\left(-m^{2}+B\right)+\frac{1}{4}(\lambda+C) \phi^{4}+\left(\frac{m^{4}}{4 \lambda}+H\right)
$$

onde A, B, C e H são contratermos. Para fazer a expansão em 1 loop em torno da 
solução clássica $\phi_{c}$ separamos os campos na forma:

$$
\phi=\phi_{c}+\Phi
$$

onde se assume um fator de $\hbar^{\frac{1}{2}}$ multiplicando $\Phi$. Analogamente, os contratermos que contribuem para a expansão em 1 loop carregam um fator de $\hbar$.

Substituindo 4.42 em 4.41 e mostrando os fatores de $\hbar$, teremos:

$$
\begin{aligned}
\mathcal{L}_{R} & =\hbar^{0}\left(-\frac{1}{2} \phi_{c} \partial_{\mu}^{2} \phi_{c}-\frac{1}{2} m^{2} \phi^{2}+\frac{\lambda}{4} \phi_{c}^{4}+\frac{m^{4}}{4 \lambda}\right) \\
& +\hbar^{1 / 2}\left(-\partial_{\mu}^{2} \phi_{c}-m^{2} \phi_{c}+\lambda \phi_{c}^{3}\right) \Phi \\
& +\hbar\left(-\frac{1}{2} \Phi \partial_{\mu}^{2} \Phi-\frac{1}{2} m^{2} \Phi^{2}+\frac{3}{2} \lambda \phi_{c}^{2} \Phi^{2}-\frac{A}{2} \phi_{c} \partial_{\mu}^{2} \phi_{c}+\frac{B}{2} \phi_{c}^{2}+\frac{C}{4} \phi_{c}^{4}+H\right) \\
& +\hbar^{3 / 2}\left(\lambda \phi_{c} \Phi^{3}-A \phi_{c} \partial_{\mu}^{2} \Phi+B \phi_{c} \Phi+C \phi_{c}^{3} \Phi\right) \\
& +\hbar^{2}\left(\frac{\lambda}{4} \Phi^{4}-\frac{A}{2} \Phi \partial_{\mu}^{2} \Phi+\frac{B}{2} \Phi^{2}+\frac{3}{2} C \phi_{c}^{2} \Phi^{2}\right)
\end{aligned}
$$

Observa-se que o termo proporcional a $\hbar^{1 / 2}$ corresponde à equação de movimento do sóliton, 2.19, e, portanto, se anula.

Para determinar os contratermos na aproximação de 1 loop impomos as seguintes condições de renormalização:

$$
\left\{\begin{array}{l}
\Gamma^{(1)}=0 \\
\Gamma^{(2)}\left(p^{2}=0\right)=2 m^{2} \\
\left.\frac{d \Gamma^{(2)}}{d p^{2}}\right|_{\left(p^{2}=0\right)}=1
\end{array}\right.
$$

Onde $\Gamma^{(1)}$ e $\Gamma^{(2)}$ são as funções de Green $1 P{ }^{4}$ de um ponto e dois pontos, respectivamente, calculadas na aproximação de 1 loop no setor do vácuo, isto é, para $\phi_{c}=\phi_{v}=m / \sqrt{\lambda}($ Veja apêndice F.1).

A função de partição bosônica na presença dos contratermos é dada por:

$$
Z\left(\phi_{c}\right)=\exp \left\{-S\left[\phi_{c}(x)\right]-S_{C T}\left(\phi_{c}\right)\right\} \int \mathcal{D} \Phi e^{-S_{B}(\Phi)}
$$

\footnotetext{
${ }^{4}$ One Particle Irreducible.
} 
onde à função de partição 4.10 foi adicionada a ação correspondente aos contratermos $S_{C T}$.

Como estamos fazendo uma aproximação semiclássica, apenas termos de $\mathcal{L}_{R}$ proporcionais a $\hbar$ são utilizados em $S_{C T}$, assim:

$$
S_{C T}\left(\phi_{c}\right)=\int d^{2} x\left[\frac{A}{2}\left(\partial_{\mu} \phi_{c}\right)^{2}+\frac{B}{2} \phi_{c}^{2}+\frac{C}{4} \phi_{c}^{4}\right]
$$

Como o que importa é a diferença entre as energias do kink e no vácuo, temos:

$$
\Gamma_{C T}=-\frac{1}{\beta} \ln \frac{Z_{C T}\left(\phi_{k}\right)}{Z_{C T}\left(\phi_{v}\right)}
$$

onde $\phi_{k}$ e $\phi_{v}$ correspondem às soluções do kink e do vácuo, respectivamente, e

$$
Z_{C T}\left(\phi_{c}\right)=e^{-S_{C T}\left(\phi_{c}\right)} .
$$

Levando a cabo esta conta, teremos:

$$
\begin{array}{r}
S_{C T}\left(\phi_{k}\right)=\int_{0}^{\beta} d \tau \int_{-\infty}^{+\infty} d x\left[\frac{A m^{4}}{4 \lambda} \operatorname{sech}^{4}(m x / \sqrt{2})+\frac{B m^{2}}{2 \lambda}\left(1-\operatorname{sech}^{2}(m x / \sqrt{2})\right)\right. \\
\left.+\frac{C m^{4}}{4 \lambda^{2}}\left(1-2 \operatorname{sech}^{2}(m x / \sqrt{2})+\operatorname{sech}^{4}(m x / \sqrt{2})\right)\right]
\end{array}
$$

$\mathrm{e}$

$$
S_{C T}\left(\phi_{v}\right)=\int_{0}^{\beta} d \tau \int_{-\infty}^{+\infty} d x\left[\frac{B}{2} \frac{m^{2}}{\lambda}+\frac{C}{4} \frac{m^{4}}{\lambda^{2}}\right] .
$$


Então:

$$
\begin{aligned}
& \Gamma_{C T}=-\frac{1}{\beta} \ln \frac{Z_{C T}\left(\phi_{k}\right)}{Z_{C T}\left(\phi_{v}\right)}=-\frac{1}{\beta} \ln \frac{e^{-S_{C T}\left(\phi_{k}\right)}}{e^{-S_{C T}\left(\phi_{v}\right)}} \\
& =-\frac{1}{\beta}\left\{-\beta \int_{-\infty}^{+\infty} d x\left[\frac{A m^{4}}{4 \lambda} \operatorname{sech}^{4}(m x / \sqrt{2})+\frac{B m^{2}}{2 \lambda}\left(1-\operatorname{sech}^{2}(m x / \sqrt{2})\right)\right.\right. \\
& \left.\left.+\frac{C m^{4}}{4 \lambda^{2}}\left(1-2 \operatorname{sech}^{2}(m x / \sqrt{2})+\operatorname{sech}^{4}(m x / \sqrt{2})\right)\right]+\beta \int_{-\infty}^{+\infty} d x\left[\frac{B m^{2}}{2 \lambda}+\frac{C m^{4}}{4 \lambda^{2}}\right]\right\} \\
& =\int_{-\infty}^{+\infty} d x\left[\frac{A m^{4}}{4 \lambda} \operatorname{sech}^{4}(m x / \sqrt{2})-\frac{B m^{2}}{2 \lambda} \operatorname{sech}^{2}(m x / \sqrt{2})-\frac{C m^{4}}{2 \lambda^{2}} \operatorname{sech}^{2}(m x / \sqrt{2})\right. \\
& \left.+\frac{C m^{4}}{4 \lambda^{2}} \operatorname{sech}^{4}(m x / \sqrt{2})\right] \\
& \Rightarrow \Gamma_{C T}=A \frac{\sqrt{2} m^{3}}{3 \lambda}-B \frac{m \sqrt{2}}{\lambda}-C \frac{2 \sqrt{2} m^{3}}{3 \lambda^{2}} .
\end{aligned}
$$

Substituindo os valores de A, B e C (Veja apêndice F.1), teremos ${ }^{5}$ :

$$
\Gamma_{C T}=3 \sqrt{2} m \int \frac{d^{2} k}{(2 \pi)^{2}}[\underbrace{\frac{1}{k^{2}+2 m^{2}}}_{I_{d}}+\underbrace{\frac{m^{2}}{\left(k^{2}+2 m^{2}\right)^{2}}+\frac{2 m^{4}}{\left(k^{2}+2 m^{2}\right)^{3}}-\frac{8 m^{6}}{\left(k^{2}+2 m^{2}\right)^{4}}}_{I_{c}}] .
$$

A única integral divergente de $\Gamma_{C T}$ é a contida em $I_{d}$, as demais integrais, $I_{c}$, convergem. A seguir vamos calcular $I_{d}$ usando cut-off:

$$
\begin{aligned}
I_{d} & =\int \frac{d^{2} k}{(2 \pi)^{2}} \frac{1}{k^{2}+2 m^{2}}=\int \frac{d k}{(2 \pi)^{2}} \int_{-\infty}^{+\infty} d k_{0} \frac{1}{k_{0}^{2}+\vec{k}^{2}+2 m^{2}} \\
& =\int \frac{d k}{(2 \pi)^{2}}\left[\frac{1}{\sqrt{\vec{k}^{2}+2 m^{2}}} \arctan \left(\frac{k_{0}}{\sqrt{\vec{k}^{2}+2 m^{2}}}\right)\right]_{-\infty}^{+\infty}=\int_{-\Lambda}^{+\Lambda} \frac{d k}{(2 \pi)^{2}} \frac{\pi}{\sqrt{\vec{k}^{2}+2 m^{2}}} \\
& =\int_{0}^{\Lambda} \frac{d k}{2 \pi} \frac{1}{\sqrt{\vec{k}^{2}+2 m^{2}}}=\frac{1}{2 \pi}\left[\ln \Lambda+\ln 2-\ln \sqrt{2 m^{2}}\right] .
\end{aligned}
$$

${ }^{5}$ Neste caso, o termo $k^{2}$ corresponde a $k^{2}=k_{0}^{2}+\vec{k}^{2}$, sendo $k_{0}$ a parte temporal e $\vec{k}$ a parte espacial do momento. 
A massa do sóliton, a temperatura zero, resulta em:

$$
M_{c}=M_{c l}+\Gamma_{B}(0)+\Gamma_{C T}=\frac{2 \sqrt{2} m^{3}}{3 \lambda}+\underbrace{\Gamma_{B}(0)+\Gamma_{C T}}_{\epsilon_{B}} .
$$

Então:

$$
\begin{aligned}
\epsilon_{B}= & \frac{m}{2} \sqrt{\frac{3}{2}}-\frac{m \sqrt{2}}{2 \pi}\left(\sqrt{3} \arctan (\sqrt{3})+3 \ln \Lambda+3 \ln 2-3 \ln \sqrt{2 m^{2}}\right) \\
& +m 3 \sqrt{2}\left[\frac{1}{2 \pi}\left(\ln \Lambda+\ln 2-\ln \sqrt{2 m^{2}}\right)+I_{c}\right] .
\end{aligned}
$$

Observa-se que as divergências contidas em $\ln \Lambda$ se anulam. No fim, obtem-se:

$$
\epsilon_{B}=\frac{m}{2} \sqrt{\frac{3}{2}}+\frac{\sqrt{2} m}{16 \pi}(5-8 \sqrt{3})=\left(1-\frac{2}{\pi}\right) \frac{\sqrt{6} m}{4}+\frac{5 \sqrt{2} m}{16 \pi} .
$$

Portanto, a massa corrigida é dada por:

$$
\begin{aligned}
M_{c} & =\frac{2 \sqrt{2} m^{3}}{3 \lambda}+\left(1-\frac{2}{\pi}\right) \frac{\sqrt{6} m}{4}+\frac{5 \sqrt{2} m}{16 \pi} \\
& \approx \frac{2 \sqrt{2} m^{3}}{3 \lambda}+0,36 m
\end{aligned}
$$

Observa-se que a correção quântica aumenta o valor da massa a temperatura zero.

\subsection{Efeitos térmicos}

Como vimos na seção 4.2, as flutuações bosônicas possuem uma parte dependente da temperatura $T$ (Veja 4.36) dada por ${ }^{6}$ :

\footnotetext{
${ }^{6}$ Observe que o valor $\beta m$ é um adimensional.
} 


$$
\begin{aligned}
\Gamma_{B}(T)= & -\frac{1}{\beta} \ln 2+\frac{1}{\beta} \ln \left(1-e^{-\beta \sqrt{3 m^{2} / 2}}\right) \\
& -\frac{m \sqrt{2}}{2 \pi} \int_{-\infty}^{+\infty} d k \frac{1}{\beta} \frac{\ln \left(1-e^{-\beta \sqrt{k^{2}+2 m^{2}}}\right)}{k^{2}+m^{2} / 2}-\frac{m \sqrt{2}}{\pi} \int_{-\infty}^{+\infty} d k \frac{1}{\beta} \frac{\ln \left(1-e^{-\beta \sqrt{k^{2}+2 m^{2}}}\right)}{k^{2}+2 m^{2}} \\
= & -\frac{1}{\beta} \ln 2+\frac{1}{\beta} \ln \left(1-e^{-\sqrt{3 m^{2} \beta^{2} / 2}}\right) \\
& -m \beta \frac{\sqrt{2}}{2 \pi} \int_{-\infty}^{+\infty} d k \frac{\ln \left(1-e^{-\sqrt{k^{2} \beta^{2}+2 m^{2} \beta^{2}}}\right)}{k^{2} \beta^{2}+m^{2} \beta^{2} / 2}-m \beta \frac{\sqrt{2}}{\pi} \int_{-\infty}^{+\infty} d k \frac{\ln \left(1-e^{-\sqrt{k^{2} \beta^{2}+2 m^{2} \beta^{2}}}\right)}{k^{2} \beta^{2}+2 m^{2} \beta^{2}}
\end{aligned}
$$

onde $\beta=1 / T$.

Vamos ver como a contribuição bosônica se comporta a altas temperaturas. Para $T \rightarrow \infty, 4.57$ se torna:

$$
\begin{aligned}
\Gamma_{B(T \rightarrow \infty)} & =-\frac{1}{\beta} \ln 2+\frac{1}{\beta} \ln \left[1-\left(1-\sqrt{3 m^{2} \beta^{2} / 2}\right)\right] \\
& -m \beta \frac{\sqrt{2}}{2 \pi} \int_{-\infty}^{+\infty} d k \frac{\ln \left(1-e^{-\sqrt{k^{2} \beta^{2}+2 m^{2} \beta^{2}}}\right)}{k^{2} \beta^{2}+m^{2} \beta^{2} / 2}-m \beta \frac{\sqrt{2}}{\pi} \int_{-\infty}^{+\infty} d k \frac{\ln \left(1-e^{-\sqrt{k^{2} \beta^{2}+2 m^{2} \beta^{2}}}\right)}{k^{2} \beta^{2}+2 m^{2} \beta^{2}} \\
& =-\frac{1}{\beta} \ln 2+\frac{1}{\beta}[\ln (\sqrt{3 / 2})+\ln (m \beta)] \\
& -m \beta \frac{\sqrt{2}}{2 \pi} \underbrace{\int_{0}^{\infty} d k \frac{\ln \left(1-e^{-\sqrt{k^{2} \beta^{2}+2 m^{2} \beta^{2}}}\right)}{k^{2} \beta^{2}+m^{2} \beta^{2} / 2}}_{B_{1}}-m \beta \frac{\sqrt{2}}{\pi} \underbrace{2 \int_{0}^{\infty} d k \frac{\ln \left(1-e^{-\sqrt{k^{2} \beta^{2}+2 m^{2} \beta^{2}}}\right)}{k^{2} \beta^{2}+2 m^{2} \beta^{2}}}_{B_{2}}
\end{aligned}
$$

Repare que para $T \rightarrow \infty$ apenas o segundo termo de 4.57 teve a exponencial aproximada da seguinte forma:

Para $x<<1$ e $x \rightarrow 0 \Rightarrow e^{x} \approx x+1+\ldots$ 
No caso das integrais $B_{1}$ e $B_{2}$ esta aproximação não pode ser feita. Por um lado $\beta \rightarrow 0$ para $T \rightarrow \infty$, no entanto, a variável $k$ contida na exponencial está sendo integrada de zero a infinito, o que não torna possível fazer a mesma aproximação.

Façamos em $B_{1}$ e $B_{2}$ a seguinte mudança de variável:

$$
x=k \beta ; \quad d x=d k \beta ; \quad y=2 m^{2} \beta^{2}
$$

teremos:

$$
\begin{aligned}
\Gamma_{B(T \rightarrow \infty)} & =-\frac{1}{\beta} \ln 2+\frac{1}{\beta}[\ln (\sqrt{3 / 2})+\ln (m \beta)] \\
& -m \frac{\sqrt{2}}{\pi} \underbrace{\int_{0}^{\infty} d x \frac{\ln \left(1-e^{-\sqrt{x^{2}+y^{2}}}\right)}{x^{2}+y^{2} / 4}}_{B_{1}}-m \frac{\sqrt{2}}{\pi} \underbrace{\int_{0}^{\infty} d x \frac{\ln \left(1-e^{-\sqrt{x^{2}+y^{2}}}\right)}{x^{2}+y^{2}}}_{B_{2}} .
\end{aligned}
$$

Ou seja:

$$
\begin{aligned}
\Gamma_{B(T \rightarrow \infty)} & =-\frac{1}{\beta} \ln 2+\frac{1}{\beta}[\ln (\sqrt{3 / 2})+\ln (m \beta)]-m \frac{\sqrt{2}}{\pi}\left(C_{1}+C_{2}\right) \\
& =\frac{1}{\beta}[\ln (\sqrt{3 / 8})+\ln (m \beta)]-m C_{B} .
\end{aligned}
$$

Sabemos que $1 / \beta$ possui dimensão de massa. Temos então um resultado dado por uma constante com dimensão de massa, tal como ocorre para o caso a temperatura zero. 



\section{Modelo $\lambda \phi^{4}$ acoplado a campo Fermiônico}

No capítulo anterior determinamos as flutuações quânticas em torno do kink do modelo $\lambda \phi^{4}$. Neste capítulo, faremos o cálculo acoplando ao sistema um campo fermiônico em duas dimensões. Na seção 5.1 é apresentado o panorama geral de como funciona a teoria de temperatura finita para o caso fermiônico. Em 5.2 é feita a quantização do modelo $\lambda \phi^{4}$ acoplado a campo fermiônico. As flutuações fermiônicas são determinadas em 5.3. Na seção 5.4 fazemos a renormalização das flutuações e a correção a temperatura zero da massa. Finalmente, na seção 5.5, determinamos as flutuações para uma temperatura finita.

\subsection{Férmions a temperatura finita}

Em Teoria de Campos, em 1+1 dimensões, os campos fermiônicos são descritos por espinores de duas componentes $\Psi_{\alpha}$ onde $\alpha=1,2$.

A densidade lagrangiana para um férmion sem massa, no espaço euclidiano, é dada por:

$$
\mathcal{L}_{F}=-\bar{\Psi}(\not \partial+i g \phi) \Psi
$$

onde $\not \partial=\gamma^{\mu} \partial_{\mu}$ e $\bar{\Psi}=\Psi^{\dagger} \gamma^{0}$. As matrizes de Dirac euclidianas $\gamma_{E}^{\mu}$ são definidas em termos das de Minkowski por: 


$$
\gamma_{E}^{\mu}=\left(\vec{\gamma}_{M}, i \gamma_{M}^{0}\right) ; \mu=1,2
$$

e satisfazem a relação de anticomutação:

$$
\left\{\gamma_{E}^{\mu}, \gamma_{E}^{\rho}\right\}=-2 \delta^{\mu \rho}
$$

Definimos as matrizes da seguinte forma:

$$
\gamma_{E}^{0}=i \sigma_{2}=\left(\begin{array}{cc}
0 & 1 \\
-1 & 0
\end{array}\right)
$$

e

$$
\gamma_{E}^{1}=i \sigma_{3}=\left(\begin{array}{cc}
i & 0 \\
0 & -i
\end{array}\right)
$$

onde $\sigma_{2}$ e $\sigma_{3}$ correspondem às matrizes de Pauli.

A função de partição correspondente (Veja 3.36) a $\mathcal{L}_{F}$ é dada por:

$$
\begin{aligned}
Z_{F} & =\int \mathcal{D} \pi \int \mathcal{D} \Psi \exp \left[-\int_{0}^{\beta} d \tau \int d x \mathcal{L}_{F}\right] \\
& =\int \mathcal{D} \Psi^{\dagger} \int \mathcal{D} \Psi \exp \left[\int_{0}^{\beta} d \tau \int d x \Psi^{\dagger}\left[\gamma_{0}\left(\not \partial+i g \phi_{c}\right)\right] \Psi\right]
\end{aligned}
$$

\subsubsection{Antiperiodicidade}

Consideremos uma onda plana fermiônica:

$$
\Psi_{\alpha}(\mathbf{x}, \tau)=e^{i\left(\mathbf{k} \cdot \mathbf{x}+\omega_{n} \tau\right)} \tilde{\Psi}_{\alpha ; n}(\mathbf{k})
$$

Neste caso, o produto ordenado é definido por:

$$
T\left[\hat{\Psi}\left(\tau_{1}\right) \hat{\Psi}\left(\tau_{2}\right)\right]=\theta\left(\tau_{1}-\tau_{2}\right) \hat{\Psi}\left(\tau_{1}\right) \hat{\Psi}\left(\tau_{2}\right)-\theta\left(\tau_{2}-\tau_{1}\right) \hat{\Psi}\left(\tau_{2}\right) \hat{\Psi}\left(\tau_{1}\right)
$$

Considerando a função de Green térmica introduzida na seção 3.2.1 e seguindo os 
mesmos passos de 3.41, chega-se a:

$$
G_{F}(x, y ; \tau, 0)=-G_{F}(x, y ; \tau, \beta)
$$

o que implica em:

$$
\Psi(x, 0)=-\Psi(x, \beta)
$$

e, portanto:

$$
\omega_{n}=(2 n+1) \pi / \beta
$$

A antiperiodicidade requerida por campos fermiônicos não torna inconsistente a operação de traço na função de partição (Veja seção 3.2). Como o sinal de $\Psi$ não é um observável, o lado direito de 5.8 descreve o mesmo estado do lado esquerdo.

\subsection{Quantização do modelo $\lambda \phi^{4}$ acoplado a campo Fermiônico}

A densidade lagrangiana do modelo $\lambda \phi^{4}$ acoplado a um campo fermiônico é dada por:

$$
\begin{aligned}
\mathcal{L} & =\frac{1}{2}\left(\partial_{\mu} \phi\right)^{2}+\frac{\lambda}{4}\left(\phi^{2}-\frac{m^{2}}{\lambda}\right)^{2}+\mathcal{L}_{F} \\
& =\frac{1}{2}\left(\partial_{\mu} \phi\right)^{2}+\frac{\lambda}{4}\left(\phi^{2}-\frac{m^{2}}{\lambda}\right)^{2}-\bar{\Psi} \not \partial \Psi-i g \bar{\Psi} \Psi \phi .
\end{aligned}
$$

Então, a função de partição $Z$ correspondente é dada por:

$$
\begin{aligned}
Z\left(\phi_{c}\right) & =\int \mathcal{D} \phi \mathcal{D} \Psi^{\dagger} \mathcal{D} \Psi e^{-S\left(\phi, \Psi, \Psi^{\dagger}\right)} \\
& =e^{-S\left[\phi_{c}(x)\right]} \int \mathcal{D} \Phi e^{-S_{B}(\Phi)} \int \mathcal{D} \Psi^{\dagger} \mathcal{D} \Psi e^{-S_{F}\left(\Psi, \Psi^{\dagger}\right)},
\end{aligned}
$$

onde foi agregado a 4.10 a interação com o campo fermiônico 5.4 e

$$
S_{F}\left(\Psi, \Psi^{\dagger}\right)=\int d^{2} x \Psi^{\dagger} \hat{\Omega}_{F}\left(\phi_{c}\right) \Psi
$$


com

$$
\hat{\Omega}_{F}\left(\phi_{c}\right)=\gamma_{0}\left(\not \partial+i g \phi_{c} I\right)
$$

onde $I$ é a matriz identidade $2 \times 2$.

Os autovalores do operador $\hat{\Omega}_{F}\left(\phi_{c}\right)$ representam as flutuações fermiônicas em torno da solução clássica $\phi_{c}$.

Analogamente ao que foi feito para o caso dos bósons, vamos determinar a diferença das flutuações fermiônicas na presença do kink e no vácuo através de:

$$
\Gamma_{F}=-\frac{1}{\beta} \ln \frac{Z_{F}\left(\phi_{k}\right)}{Z_{F}\left(\phi_{v}\right)}
$$

onde (Veja apêndice A.2):

$$
Z_{F}\left(\phi_{k}\right)=\int \mathcal{D} \Psi^{\dagger} \mathcal{D} \Psi \exp \left\{\int d^{2} x\left[\Psi^{\dagger} \hat{\Omega}_{F}\left(\phi_{k}\right) \Psi\right]\right\}=C\left[\operatorname{det} \hat{\Omega}_{F}\left(\phi_{k}\right)\right]
$$

e

$$
Z_{F}\left(\phi_{v}\right)=\int \mathcal{D} \Psi^{\dagger} \mathcal{D} \Psi \exp \left\{-\int d^{2} x\left[\Psi^{\dagger} \hat{\Omega}_{F}\left(\phi_{v}\right) \Psi\right]\right\}=C\left[\operatorname{det} \hat{\Omega}_{F}\left(\phi_{v}\right)\right]
$$

Assim:

$$
\begin{aligned}
\Gamma_{F} & =-\frac{1}{\beta}\left\{\ln Z_{F}\left(\phi_{k}\right)-\ln Z_{F}\left(\phi_{v}\right)\right\} \\
& =-\frac{1}{\beta}\left\{\ln C+\ln \left[\operatorname{det} \hat{\Omega}_{F}\left(\phi_{k}\right)\right]-\ln C-\ln \left[\operatorname{det} \hat{\Omega}_{F}\left(\phi_{v}\right)\right]\right\} \\
& =-\frac{1}{\beta}\left\{\ln \left[\operatorname{det} \hat{\Omega}_{F}\left(\phi_{k}\right)\right]-\ln \left[\operatorname{det} \hat{\Omega}_{F}\left(\phi_{v}\right)\right]\right\} .
\end{aligned}
$$

Usando a identidade $\ln \operatorname{det} \hat{A}=\operatorname{tr} \ln \hat{A}$ (Veja apêndice B), teremos:

$$
\Gamma_{F}=-\frac{1}{\beta}\left\{\operatorname{tr} \ln \hat{\Omega}_{F}\left(\phi_{k}\right)-\operatorname{tr} \ln \hat{\Omega}_{F}\left(\phi_{v}\right)\right\}
$$

Comparando esta expressão com o caso bosônico, 4.17, vemos que ao invés do fator $1 / 2$ temos -1 , isto se deve aos dois graus de liberdade dos férmions. 


\subsection{Cálculo das flutuações fermiônicas}

Nesta seção vamos determinar o valor de 5.18, isto é, a contribuição das flutuações fermiônicas à massa corrigida do sóliton, dada por:

$$
M_{c}=M_{c l}+\Gamma_{B}+\Gamma_{F}=\frac{2 \sqrt{2} m^{3}}{3 \lambda}+\Gamma_{B}+\Gamma_{F},
$$

onde à 4.16 foi somada a contribuição fermiônica $\Gamma_{F}$.

O operador fermiônico é dado por 5.13:

$$
\hat{\Omega}_{F}\left(\phi_{c}\right)=\gamma_{0}\left(\not \partial+i g \phi_{c} I\right)
$$

onde $I$ é a matriz identidade $2 \times 2$. Os valores assumidos por $\phi_{c}(x)$ correspondentes ao kink e ao vácuo são dados, respectivamente, por:

$$
\phi_{k}(x)=(m / \sqrt{\lambda}) \tanh (m x / \sqrt{2})
$$

$\mathrm{e}$

$$
\phi_{v}(x)=m / \sqrt{\lambda}
$$

Para determinar 5.18 temos que calcular $\operatorname{det} \hat{\Omega}_{F}\left(\phi_{c}\right)$, então:

$$
\begin{aligned}
\operatorname{det} \hat{\Omega}_{F}\left(\phi_{c}\right) & =\operatorname{det}\left[\gamma_{0}\left(\not \partial+i g \phi_{c}(x) I\right)\right]=\left(\operatorname{det} \gamma_{0}\right) \operatorname{det}\left(\not \partial+i g \phi_{c}(x) I\right) \\
& =\operatorname{det}\left(\not \partial+i g \phi_{c}(x) I\right)
\end{aligned}
$$

onde $\operatorname{det} \gamma_{0}=1$ e $I$ é a matriz identidade $2 \times 2$.

Vamos chamar $\phi(x)=i g \phi_{c}(x)$ para simplificar. A conjugação de carga (Veja apendice D) nos permite escrever: 


$$
\begin{aligned}
\operatorname{det}(\not \partial+\phi(x) I) & =\operatorname{det}^{1 / 2}(\not \partial+\phi(x) I) \operatorname{det}^{1 / 2}(\not \partial-\phi(x) I) \\
& =\operatorname{det}^{1 / 2}[(\not \partial+\phi(x) I)(\not \partial-\phi(x) I)] \\
& =\operatorname{det}^{1 / 2}\left[\left(-\partial_{0}^{2}-\partial_{x}^{2}-\phi(x)^{2} I\right)-\gamma_{1} \partial_{x} \phi(x)\right] \\
& =\operatorname{det}^{1 / 2}\left[\left(-\square-\phi(x)^{2} I\right)-\gamma_{1} \partial_{x} \phi(x)\right]
\end{aligned}
$$

Assim:

$$
\begin{aligned}
\operatorname{det}(\not \partial+\phi(x) I) & =\operatorname{det}^{1 / 2}\left(\begin{array}{cc}
-\square-\phi(x)^{2}-i \partial_{x} \phi(x) & 0 \\
0 & -\square-\phi(x)^{2}+i \partial_{x} \phi(x)
\end{array}\right) \\
& =\operatorname{det}^{1 / 2}\left(-\square-\phi(x)^{2}-i \partial_{x} \phi(x)\right) \operatorname{det}^{1 / 2}\left(-\square-\phi(x)^{2}+i \partial_{x} \phi(x)\right) .
\end{aligned}
$$

Para o caso do vácuo, temos:

$$
\phi(x)=i g \phi_{v}=i g \frac{m}{\sqrt{\lambda}} .
$$

Temos que $\phi^{\prime}(x)=0$ e 5.25 fica:

$$
\operatorname{det} \hat{\Omega}_{F}\left(\phi_{v}\right)=\operatorname{det}\left(\not \partial+i g \phi_{v} I\right)=\operatorname{det}\left(-\square+g^{2} \phi_{v}^{2}\right)
$$

As autofunções deste operador são ondas planas antiperiódicas no tempo euclidiano dadas por:

$$
\Psi_{\alpha}(\mathbf{x}, \tau)=e^{i\left(\mathbf{k x}+\omega_{n} \tau\right)} \tilde{\Psi}_{\alpha ; n}(\mathbf{k})
$$

Assim, os autovalores de $\hat{\Omega}_{F}\left(\phi_{v}\right)$ são dados por:

$$
\begin{array}{r}
\hat{\Omega}_{F}\left(\phi_{v}\right) \Psi_{\alpha}(\mathbf{x}, \tau)=\varepsilon_{F}\left(\phi_{v}\right) \Psi_{\alpha}(\mathbf{x}, \tau) \\
\Rightarrow \varepsilon_{F}\left(\phi_{v}\right)=\omega_{n}^{2}+\mathbf{k}^{2}+g^{2} \phi_{v}^{2} .
\end{array}
$$

Como as ondas devem ser antiperiódicas no tempo (com um período $\beta$ ), temos: 


$$
\begin{aligned}
& \Psi(\mathbf{x}, \tau+\beta)=-\Psi(\mathbf{x}, \beta) \\
& \Rightarrow e^{i \omega_{n}(\tau+\beta)}=-e^{i \omega \tau} e^{i 2 \pi n},
\end{aligned}
$$

então: $\omega_{n} \beta=(2 n+1) \pi(n=0, \pm 1, \pm 2, \ldots)$.

Para o caso do kink, temos:

$$
\phi(x)=i g \phi_{k}(x)=i g \frac{m}{\sqrt{\lambda}} \tanh \left(\frac{m x}{\sqrt{2}}\right) .
$$

Substituindo em 5.25:

$$
\begin{aligned}
\operatorname{det} \hat{\Omega}_{F}\left(\phi_{k}\right) & =\operatorname{det}\left(\not \partial+i g \phi_{k}(x) I\right) \\
& =\operatorname{det}^{1 / 2}\left(-\square+g^{2} \phi_{k}(x)^{2}+g \partial_{x} \phi_{k}(x)\right) \operatorname{det}^{1 / 2}\left(-\square+g^{2} \phi_{k}(x)^{2}-g \partial_{x} \phi_{k}(x)\right) \\
& =\operatorname{det}^{1 / 2} \tilde{\Theta}^{(+)}\left(\phi_{k}\right) \operatorname{det}^{1 / 2} \tilde{\Theta}^{(-)}\left(\phi_{k}\right) .
\end{aligned}
$$

Temos, então:

$$
\begin{aligned}
\tilde{\Theta}^{( \pm)}\left(\phi_{k}\right) & =-\square+g^{2} \phi_{k}(x)^{2} \pm g \partial_{x} \phi_{k}(x) \\
& =-\square+g^{2} \frac{m^{2}}{\lambda} \tanh ^{2}(m x / \sqrt{2}) \pm g \frac{m^{2}}{\sqrt{2 \lambda}} \operatorname{sech}^{2}(m x / \sqrt{2}) \\
& =-\square+g^{2} \frac{m^{2}}{\lambda}\left(1-\operatorname{sech}^{2}(m x / \sqrt{2}) \pm g \frac{m^{2}}{\sqrt{2 \lambda}} \operatorname{sech}^{2}(m x / \sqrt{2})\right. \\
& =-\square+m^{2}\left[\frac{g^{2}}{\lambda}+\operatorname{sech}^{2}(m x / \sqrt{2})\left(-\frac{g^{2}}{\lambda} \pm \frac{g}{\sqrt{2 \lambda}}\right)\right] .
\end{aligned}
$$

Definindo o parâmetro $S$ como:

$$
S^{2}=2 g^{2} / \lambda
$$

obtemos:

$$
\tilde{\Theta}^{( \pm)}\left(\phi_{k}\right)=-\square+\frac{m^{2}}{2}\left[S^{2}-S(S \pm 1) \operatorname{sech}^{2}(m x / \sqrt{2})\right] .
$$

Então: 


$$
\left[-\square+\frac{m^{2}}{2}\left[S^{2}-S(S \pm 1) \operatorname{sech}^{2}(m x / \sqrt{2})\right]\right] \Psi(x, \tau)=\varepsilon_{F}^{ \pm}\left(\phi_{k}\right) \Psi(x, \tau) .
$$

Se tentarmos uma solução da forma:

$$
\Psi(x, \tau)=e^{i \omega_{n} \tau} \Psi(x),
$$

temos para a equação puramente espacial:

$$
\left[-\partial_{x}^{2}+\frac{m^{2}}{2}\left[S^{2}-S(S \pm 1) \operatorname{sech}^{2}(m x / \sqrt{2})\right]\right] \Psi(x)=\left(\varepsilon_{F}^{ \pm}\left(\phi_{k}\right)-\omega_{n}^{2}\right) \Psi(x) .
$$

Para determinar os autovalores dessa equação devemos proceder de maneira análoga ao caso puramente bosônico, observando que esta equação é semelhante à equação de Schröedinger com o potencial de Poschl-Teller, resolvido em detalhe em [17]. Vamos nos restringir aos casos em que $S$ é um inteiro $(S=1,2,3, \ldots)$. Nesse caso as soluções são não-reflexivas e dadas por uma série de níveis discretos e um contínuo.

Os resultados para o operador $\tilde{\Theta}^{(+)}\left(\phi_{k}\right)$ são:

$$
\varepsilon^{(+)}\left(\phi_{k}\right)=\left\{\begin{array}{l}
\omega_{n}^{2}+m^{2} l\left(S-\frac{l}{2}\right) ; \quad l=0,1, \ldots, S-1 \\
\omega_{n}^{2}+k^{2}+\underbrace{\frac{1}{2} m^{2} S^{2}}_{M^{2}}
\end{array}\right.
$$

com o deslocamento de fase dos estados espalhados dado por [2] e [7]:

$$
\delta^{+}(k)=\pi S-2 \sum_{l=0}^{S-1} \arctan \left(\frac{\sqrt{2} k}{m(l+1)}\right) .
$$

Para o caso do operador $\tilde{\Theta}^{(-)}\left(\phi_{k}\right)$, temos:

$$
\varepsilon^{(-)}\left(\phi_{k}\right)=\left\{\begin{array}{l}
\omega_{n}^{2}+m^{2}(l+1)\left(S-\frac{l+1}{2}\right) ; \quad l=0,1, \ldots, S-2 \\
\omega_{n}^{2}+k^{2}+\underbrace{\frac{1}{2} m^{2} S^{2}}_{M^{2}}
\end{array}\right.
$$


com o deslocamento de fase dado por:

$$
\delta^{-}(k)=\pi(S-1)-2 \sum_{l=0}^{S-2} \arctan \left(\frac{\sqrt{2} k}{m(l+1)}\right) .
$$

O resultado $m^{2} S^{2} / 2$, que aparece no contínuo de 5.39 e 5.41 , corresponde ao quadrado da massa da flutuação fermiônica $M^{2}=m^{2} S^{2} / 2$. A partir de agora utilizaremos esta notação.

Determinados os autovalores do operador fermiônico no vácuo e na presença do kink, podemos prosseguir com o cálculo das flutuações fermiônicas 5.18. Usando 5.29, 5.39 e $5.41:$

$$
\begin{aligned}
\Gamma_{F} & =-\frac{1}{\beta}\left\{t r \ln \hat{\Omega}_{F}\left(\phi_{k}\right)-\operatorname{tr} \ln \hat{\Omega}_{F}\left(\phi_{v}\right)\right\} \\
& =-\frac{1}{\beta}\left[\frac{1}{2} \operatorname{tr} \ln \hat{\Theta}^{(+)}+\frac{1}{2} \operatorname{tr} \ln \hat{\Theta}^{(-)}-t r \ln \hat{\Omega}_{F}\left(\phi_{v}\right)\right] \\
& =-\frac{1}{\beta}\left(\Gamma_{F 1}+\Gamma_{F 2}-\Gamma_{F 3}\right) .
\end{aligned}
$$

Temos:

$$
\begin{aligned}
& \Gamma_{F 1}= \frac{1}{2} \operatorname{tr} \ln \hat{\Theta}^{(+)} \\
&= \frac{1}{2}\left[\sum_{n} \sum_{l=0}^{S-1} \ln \left(\omega_{n}^{2}+m^{2} l(S-l / 2)\right)+\sum_{n} \sum_{k} \ln \left(\omega_{n}^{2}+k^{2}+M^{2}\right)\right] \\
&=\frac{1}{2}\left[\sum_{n} \sum_{l=0}^{S-1} \ln \left(\omega_{n}^{2}+m^{2} l(S-l / 2)\right)+\sum_{n} \frac{L}{2 \pi} \int d k\left(1+\frac{1}{L} \frac{d \delta^{+}}{d k}\right) \ln \left(\omega_{n}^{2}+k^{2}+M^{2}\right)\right] \\
& \Gamma_{F 2}=\frac{1}{2} \operatorname{tr} \ln \hat{\Theta}^{(-)}=\frac{1}{2}\left[\sum_{n} \sum_{l=0}^{S-2} \ln \left(\omega_{n}^{2}+m^{2}(l+1)\left(S-\frac{l+1}{2}\right)\right)\right. \\
&\left.\quad+\sum_{n} \frac{L}{2 \pi} \int d k\left(1+\frac{1}{L} \frac{d \delta^{-}}{d k}\right) \ln \left(\omega_{n}^{2}+k^{2}+M^{2}\right)\right]
\end{aligned}
$$


$\Gamma_{F 3}=\operatorname{tr} \ln \hat{\Omega}_{F}\left(\phi_{v}\right)=\sum_{n} \sum_{k} \ln \left(\omega_{n}^{2}+k^{2}+M^{2}\right)=\sum_{n} \int d k \frac{L}{2 \pi} \ln \left(\omega_{n}^{2}+k^{2}+M^{2}\right)$

Então:

$$
\begin{aligned}
\Gamma_{F 1}+\Gamma_{F 2}-\Gamma_{F 3} & =\frac{1}{2} \sum_{n} \sum_{l=0}^{S-1} \ln \left(\omega_{n}^{2}+m^{2} l(S-l / 2)\right)+\frac{1}{2} \sum_{n} \sum_{l=1}^{S-1} \ln \left(\omega_{n}^{2}+m^{2} l(S-l / 2)\right) \\
& +\frac{1}{2} \sum_{n} \int \frac{d k}{2 \pi} \frac{d \delta^{+}}{d k} \ln \left(\omega_{n}^{2}+k^{2}+M^{2}\right) \\
& +\frac{1}{2} \sum_{n} \int \frac{d k}{2 \pi} \frac{d \delta^{-}}{d k} \ln \left(\omega_{n}^{2}+k^{2}+M^{2}\right) \\
& =\frac{1}{2} \sum_{n} \sum_{l=0}^{S-1} \ln \left(\omega_{n}^{2}+m^{2} l(S-l / 2)\right)+\frac{1}{2} \sum_{n} \sum_{l=1}^{S-1} \ln \left(\omega_{n}^{2}+m^{2} l(S-l / 2)\right) \\
& -\sqrt{2} \sum_{n} \sum_{l=0}^{S-1} \int \frac{d k}{2 \pi} \frac{m(l+1)}{\left(m^{2}(l+1)^{2}+2 k^{2}\right)} \ln \left(\omega_{n}^{2}+k^{2}+M^{2}\right) \\
& -\sqrt{2} \sum_{n} \sum_{l=0}^{S-2} \int \frac{d k}{2 \pi} \frac{m(l+1)}{\left(m^{2}(l+1)^{2}+2 k^{2}\right)} \ln \left(\omega_{n}^{2}+k^{2}+M^{2}\right)
\end{aligned}
$$

Utilizando a identidade (Veja apêndice C.2)

$$
\sum_{n=-\infty}^{+\infty} \ln \left(\alpha^{2}+\omega_{n}^{2}\right)=\beta \alpha+2 \ln \left(1+e^{-\beta \alpha}\right)+\mathcal{C}
$$

obtem-se: 


$$
\begin{aligned}
\Gamma_{F} & =-\frac{1}{2} \sum_{l=0}^{S-1}\left[\sqrt{m^{2} l(S-l / 2)}+\frac{2}{\beta} \ln \left(1+e^{-\beta \sqrt{m^{2} l(S-l / 2)}}\right)\right] \\
& -\frac{1}{2} \sum_{l=1}^{S-1}\left[\sqrt{m^{2} l(S-l / 2)}+\frac{2}{\beta} \ln \left(1+e^{-\beta \sqrt{m^{2} l(S-l / 2)}}\right)\right] \\
& +\sqrt{2} m \sum_{l=0}^{S-1} \int \frac{d k}{2 \pi}\left[\frac{l+1}{m^{2}(l+1)^{2}+2 k^{2}}\right]\left[\sqrt{k^{2}+M^{2}}+\frac{2}{\beta} \ln \left(1+e^{-\beta \sqrt{k^{2}+M^{2}}}\right)\right] \\
& +\sqrt{2} m \sum_{l=0}^{S-2} \int \frac{d k}{2 \pi}\left[\frac{l+1}{m^{2}(l+1)^{2}+2 k^{2}}\right]\left[\sqrt{k^{2}+M^{2}}+\frac{2}{\beta} \ln \left(1+e^{-\beta \sqrt{k^{2}+M^{2}}}\right)\right]
\end{aligned}
$$

A parte independente da temperatura é dada por:

$$
\begin{aligned}
\Gamma_{F}(0)= & -\sum_{l=1}^{S-1} \sqrt{m^{2} l(S-l / 2)}+\sqrt{2} m \sum_{l=0}^{S-1} \int \frac{d k}{2 \pi}\left[\frac{l+1}{m^{2}(l+1)^{2}+2 k^{2}}\right] \sqrt{k^{2}+M^{2}} \\
& +\sqrt{2} m \sum_{l=0}^{S-2} \int \frac{d k}{2 \pi}\left[\frac{l+1}{m^{2}(l+1)^{2}+2 k^{2}}\right] \sqrt{k^{2}+M^{2}}
\end{aligned}
$$

Como podemos observar, este resultado possui integrais divergentes que precisam ser renormalizadas. Na próxima seção veremos como fazer isso.

\subsection{Correção da massa a temperatura zero}

A densidade lagrangiana renormalizada do modelo $\lambda \phi^{4}$ acoplado a um campo fermiônico é dada por:

$$
\begin{aligned}
\mathcal{L}_{R}= & \frac{1}{2}(1+A)\left(\partial_{\mu} \phi\right)^{2}+\frac{1}{2}\left(-m^{2}+B\right) \phi^{2}+\frac{1}{4}(\lambda+C) \phi^{4}+ \\
& -(1+F) \bar{\Psi} \not \partial \Psi-i(g+G) \bar{\Psi} \Psi \phi+\left(\frac{m^{4}}{4 \lambda}+H\right),
\end{aligned}
$$

onde A, B, C, F, G e H são contratermos. Analogamente ao que foi feito para o caso puramente bosônico, faremos a aproximação em 1 loop em torno da solução clássica $\phi_{c}$ separando os campos da seguinte forma: 


$$
\begin{aligned}
& \phi=\phi_{c}+\Phi \\
& \Psi=\Psi
\end{aligned}
$$

onde se assume que um fator de $\hbar^{1 / 2}$ multiplica $\Phi$ e $\Psi$. Similarmente, os contratermos carregam um fator de $\hbar$.

Substituindo estas expansões em $\mathcal{L}_{R}$ :

$$
\begin{aligned}
\mathcal{L}_{R} & =\hbar^{0}\left(-\frac{1}{2} \phi_{c} \partial_{\mu}^{2} \phi_{c}-\frac{1}{2} m^{2} \phi^{2}+\frac{1}{4} \lambda \phi_{c}^{4}+\frac{m^{4}}{4 \lambda}\right) \\
& +\hbar^{1 / 2}\left(-\phi_{c} \partial_{\mu}^{2} \Phi-m^{2} \phi_{c} \Phi+\lambda \phi_{c}^{3} \Phi\right) \\
& +\hbar\left(-\frac{1}{2} \Phi \partial_{\mu}^{2} \Phi-\frac{1}{2} m^{2} \Phi^{2}+\frac{3}{2} \lambda \phi_{c}^{2} \Phi^{2}-\frac{A}{2} \phi_{c} \partial_{\mu}^{2} \phi_{c}+\frac{B}{2} \phi_{c}^{2}+\frac{C}{4} \phi_{c}^{4}+H-\bar{\Psi} \not \partial \Psi-i g \bar{\Psi} \Psi \phi_{c}\right) \\
& +\hbar^{3 / 2}\left(\lambda \phi_{c} \Phi^{3}-A \phi_{c} \partial_{\mu}^{2} \Phi+B \phi_{c} \Phi+C \phi_{c}^{3} \Phi-i g \bar{\Psi} \Psi \Phi\right) \\
& +\hbar^{2}\left(\frac{\lambda}{4} \Phi^{4}-\frac{A}{2} \Phi \partial_{\mu}^{2} \Phi+\frac{B}{2} \Phi^{2}+\frac{3}{2} C \phi_{c}^{2} \Phi^{2}-F \bar{\Psi} \not \partial \Psi-i G \bar{\Psi} \Psi \phi_{c}\right)+O\left(\hbar^{5 / 2}\right) .
\end{aligned}
$$

Para determinar os contratermos na aproximação de 1 loop impomos as seguintes condições de renormalização:

$$
\left\{\begin{array}{l}
\Gamma^{(1)}=0 \\
\Gamma^{(2)}\left(p^{2}=0\right)=2 m^{2} \\
\left.\frac{d \Gamma^{(2)}}{d p^{2}}\right|_{\left(p^{2}=0\right)}=1
\end{array}\right.
$$

onde $\Gamma^{(1)}$ e $\Gamma^{(2)}$ são as funções de Green 1PI (de um e dois pontos) calculadas na aproximação de 1 loop no setor do vácuo, isto é, para $\phi_{c}=\phi_{v}=m / \sqrt{\lambda}$ (Veja apêndice F.2).

A função de partição do modelo $\lambda \phi^{4}$ acoplado a campo fermiônico na presença dos contratermos é dada por:

$$
\begin{aligned}
Z\left(\phi_{c}\right) & =\int \mathcal{D} \phi \mathcal{D} \Psi^{\dagger} \mathcal{D} \Psi e^{-S\left(\phi, \Psi, \Psi^{\dagger}\right)} \\
& =e^{-S\left[\phi_{c}(x)\right]-S_{C T}\left(\phi_{c}\right)} \int \mathcal{D} \Phi e^{-S_{B}(\Phi)} \int \mathcal{D} \Psi^{\dagger} \mathcal{D} \Psi e^{-S_{F}\left(\Psi, \Psi^{\dagger}\right)}
\end{aligned}
$$

onde à função de partição 5.11 foi adicionada a ação correspondente aos contratermos 
$S_{C T}$, dada por 4.46 :

$$
S_{C T}\left(\phi_{c}\right)=\int d^{2} x\left[\frac{A}{2}\left(\partial_{\mu} \phi_{c}\right)^{2}+\frac{B}{2} \phi_{c}^{2}+\frac{C}{4} \phi_{c}^{4}\right]
$$

Como o que importa é a diferença entre as energias do kink e no vácuo, temos de 4.51:

$$
\Gamma_{C T}=-\frac{1}{\beta} \frac{Z_{C T}\left(\phi_{k}\right)}{Z_{C T}\left(\phi_{v}\right)}=A \frac{\sqrt{2} m^{3}}{3 \lambda}-B \frac{m \sqrt{2}}{\lambda}-C \frac{2 \sqrt{2} m^{3}}{3 \lambda^{2}}
$$

Substituindo os valores dos contratermos ${ }^{1}$ (Veja apêndice F.2), temos:

$$
\begin{aligned}
\Gamma_{C T_{F}}=A & \frac{\sqrt{2} m^{3}}{3 \lambda}-B \frac{m \sqrt{2}}{\lambda}-C \frac{2 \sqrt{2} m^{3}}{3 \lambda^{2}} \\
=-S^{2} \sqrt{2} m \int \frac{d^{2} k}{(2 \pi)^{2}}( & \frac{1}{k^{2}+M^{2}}+\frac{m^{2} S^{2}}{6} \frac{1}{\left(k^{2}+M^{2}\right)^{2}}+\frac{m^{4} S^{2}}{2} \frac{1}{\left(k^{2}+M^{2}\right)^{3}} \\
& \left.-\frac{m^{6} S^{4}}{3} \frac{1}{\left(k^{2}+M^{2}\right)^{4}}\right) .
\end{aligned}
$$

A correção da massa do sóliton a temperatura zero é dada por:

$$
M_{c}=M_{c l}+\underbrace{\Gamma_{B}(0)+\Gamma_{C T_{B}}}_{\epsilon_{B}}+\underbrace{\Gamma_{F}(0)+\Gamma_{C T_{F}}}_{\epsilon_{F}} .
$$

Onde $M_{c l}$ é o valor da massa clássica, $\Gamma_{B}(0)$ são as flutuações bosônicas já calculadas na seção $4.2, \Gamma_{F}$ são as flutuações fermiônicas e $\Gamma_{C T_{B}}$ e $\Gamma_{C T_{F}}$ são os termos correspondentes aos contratermos das partes bosônica e fermiônica, respectivamente.

Levando em consideração apenas a contribuição fermiônica, temos:

\footnotetext{
${ }^{1}$ Aqui foi substituída apenas a parte fermiônica dos contratermos
} 


$$
\begin{aligned}
\epsilon_{F} & =-\sum_{l=1}^{S-1} \sqrt{m^{2} l(S-l / 2)} \\
& +\sqrt{2} m \sum_{l=0}^{S-1} \int \frac{d k}{2 \pi}\left[\frac{(l+1) \sqrt{k^{2}+M^{2}}}{m^{2}(l+1)^{2}+2 k^{2}}\right]+\sqrt{2} m \sum_{l=0}^{S-2} \int \frac{d k}{2 \pi}\left[\frac{(l+1) \sqrt{k^{2}+M^{2}}}{m^{2}(l+1)^{2}+2 k^{2}}\right] \\
& -S^{2} \sqrt{2} m \int \frac{d^{2} k}{(2 \pi)^{2}}(\underbrace{\frac{1}{k^{2}+M^{2}}}_{I_{d}}+\underbrace{\frac{m^{2} S^{2}}{6} \frac{1}{\left(k^{2}+M^{2}\right)^{2}}+\frac{m^{4} S^{2}}{2} \frac{1}{\left(k^{2}+M^{2}\right)^{3}}-\frac{m^{6} S^{4}}{3} \frac{1}{\left(k^{2}+M^{2}\right)^{4}}}_{I_{c}}),
\end{aligned}
$$

onde $I_{d}$ é a única integral divergente dos contratermos e $I_{c}$ são os termos convergentes.

Como vimos na seção anterior, $S$ é um número inteiro positivo. A seguir, vamos calcular a contribuição fermiônica $\epsilon_{F}$ para os valores $S=1$ e $S=2$.

Para $S=1$ ou $\lambda=2 g^{2}$ (Veja 5.34), temos:

$$
\begin{aligned}
\epsilon_{F}(S=1) & =\frac{\sqrt{2} m}{4 \pi} \underbrace{\int d k \frac{\sqrt{k^{2}+M^{2}}}{k^{2}+m^{2} / 2}}_{I_{1}} \\
& -\sqrt{2} m \int \frac{d^{2} k}{(2 \pi)^{2}}(\underbrace{\frac{1}{k^{2}+M^{2}}}_{I_{d}}+\underbrace{\left.\frac{m^{2}}{6} \frac{1}{\left(k^{2}+M^{2}\right)^{2}}+\frac{m^{4}}{2} \frac{1}{\left(k^{2}+M^{2}\right)^{3}}-\frac{m^{6}}{3} \frac{1}{\left(k^{2}+M^{2}\right)^{4}}\right),}_{I_{c}}
\end{aligned}
$$

onde $M^{2}=m^{2} S^{2} / 2=m^{2} / 2$. Então:

$$
I_{1}=\int d k \frac{1}{\sqrt{k^{2}+m^{2} / 2}}=2\left(\ln \Lambda+\ln 2-\ln \sqrt{m^{2} / 2}\right)
$$

Podemos resolver $I_{d}$ por cut-off:

$$
I_{d}=\frac{d^{2} k}{(2 \pi)^{2}} \frac{1}{k^{2}+M^{2}}=\int_{-\Lambda}^{+\Lambda} \frac{d \vec{k}}{(2 \pi)^{2}} \int_{-\infty}^{+\infty} d k_{0} \frac{1}{k_{0}^{2}+\vec{k}^{2}+m^{2} / 2}=\frac{1}{2 \pi}\left(\ln \Lambda+\ln 2-\ln \sqrt{m^{2} / 2}\right)
$$

E portanto:

$$
\epsilon_{F}(S=1)=\frac{\sqrt{2} m}{2 \pi}\left(\ln \Lambda+\ln 2-\ln \sqrt{m^{2} / 2}\right)-\frac{\sqrt{2} m}{2 \pi}\left(\ln \Lambda+\ln 2-\ln \sqrt{m^{2} / 2}\right)-\sqrt{2} m I_{c} .
$$


As divergências se anulam e sobram apenas termos finitos, o resultado final é dado por:

$$
\epsilon_{F}\left(\lambda=2 g^{2}\right)=-\frac{\sqrt{2}}{9 \pi} m
$$

Neste caso, a massa do sóliton é dada por (Veja 5.58):

$$
\begin{aligned}
M_{c} & =\frac{2 \sqrt{2} m^{3}}{3 \lambda}+\left[\left(1-\frac{2}{\pi}\right) \frac{\sqrt{6}}{4}+\frac{5 \sqrt{2}}{16 \pi}-\frac{\sqrt{2}}{9 \pi}\right] m \\
& \approx \frac{2 \sqrt{2} m^{3}}{3 \lambda}+0,31 m
\end{aligned}
$$

De maneira análoga, determinamos o resultado para o caso $S=2\left(\right.$ ou $\left.\lambda=g^{2} / 2\right)$ :

$$
\epsilon_{F}\left(\lambda=g^{2} / 2\right)=-m \sqrt{\frac{3}{2}}+\frac{m \sqrt{6}}{3}-m \frac{13 \sqrt{2}}{36 \pi}=-\frac{\sqrt{2}}{36 \pi}(6 \sqrt{3} \pi+13) m .
$$

Observemos que neste caso temos $M^{2}=2 m^{2}$, ou seja, o valor da massa da flutuação fermiônica é igual ao da massa da flutuação bosônica (Veja 4.28).

A massa do sóliton é dada por:

$$
\begin{aligned}
M_{c} & =\frac{2 \sqrt{2} m^{3}}{3 \lambda}+\left[\left(1-\frac{2}{\pi}\right) \frac{\sqrt{6}}{4}+\frac{5 \sqrt{2}}{16 \pi}-\frac{\sqrt{2}}{36 \pi}(6 \sqrt{3} \pi+13)\right] m \\
& \approx \frac{2 \sqrt{2} m^{3}}{3 \lambda}-0,21 m
\end{aligned}
$$

\subsection{Efeitos térmicos}

Analogamente ao que foi feito na seção 4.4, também veremos como a contribuição fermiônica se comporta a altas temperaturas. A parte da flutuação fermiônica 5.48 dependente da temperatura $T$ é dada por ${ }^{2}$ :

\footnotetext{
${ }^{2}$ Observe que $m \beta$ e $M \beta$ são adimensionais.
} 


$$
\begin{aligned}
& \Gamma_{F}(T)=-\frac{1}{\beta} \sum_{l=0}^{S-1} \ln \left(1+e^{-\beta \sqrt{m^{2} l(S-l / 2)}}\right)-\frac{1}{\beta} \sum_{l=1}^{S-1} \ln \left(1+e^{-\beta \sqrt{m^{2} l(S-l / 2)}}\right) \\
& +\frac{m \sqrt{2}}{\pi} \sum_{l=0}^{S-1} \int_{-\infty}^{+\infty} d k\left[\frac{l+1}{m^{2}(l+1)^{2}+2 k^{2}}\right] \frac{1}{\beta} \ln \left(1+e^{-\beta \sqrt{k^{2}+M^{2}}}\right) \\
& +\frac{m \sqrt{2}}{\pi} \sum_{l=0}^{S-2} \int_{-\infty}^{+\infty} d k\left[\frac{l+1}{m^{2}(l+1)^{2}+2 k^{2}}\right] \frac{1}{\beta} \ln \left(1+e^{-\beta \sqrt{k^{2}+M^{2}}}\right) \\
& \Rightarrow \Gamma_{F}(T)=-\frac{1}{\beta} \sum_{l=0}^{S-1} \ln \left(1+e^{-\sqrt{m^{2} \beta^{2} l(S-l / 2)}}\right)-\frac{1}{\beta} \sum_{l=1}^{S-1} \ln \left(1+e^{-\sqrt{m^{2} \beta^{2} l(S-l / 2)}}\right) \\
& +m \beta \frac{\sqrt{2}}{2 \pi} \sum_{l=0}^{S-1} \int_{-\infty}^{+\infty} d k\left[\frac{l+1}{m^{2} \beta^{2}(l+1)^{2} / 2+k^{2} \beta^{2}}\right] \ln \left(1+e^{-\sqrt{k^{2} \beta^{2}+M^{2} \beta^{2}}}\right) \\
& +m \beta \frac{\sqrt{2}}{2 \pi} \sum_{l=0}^{S-2} \int_{-\infty}^{+\infty} d k\left[\frac{l+1}{m^{2} \beta^{2}(l+1)^{2} / 2+k^{2} \beta^{2}}\right] \ln \left(1+e^{-\sqrt{k^{2} \beta^{2}+M^{2} \beta^{2}}}\right)
\end{aligned}
$$

Para $T \rightarrow \infty$ esta expressão fica:

$$
\begin{aligned}
\Gamma_{F}(T \rightarrow \infty) & =-\sum_{l=0}^{S-1} \frac{1}{\beta} \ln \left(2-\sqrt{m^{2} \beta^{2} l(S-l / 2)}\right)-\frac{1}{\beta} \sum_{l=1}^{S-1} \ln \left(2-\sqrt{m^{2} \beta^{2} l(S-l / 2)}\right) \\
& +m \beta \frac{\sqrt{2}}{2 \pi} \sum_{l=0}^{S-1} \int_{-\infty}^{+\infty} d k\left[\frac{l+1}{m^{2} \beta^{2}(l+1)^{2} / 2+k^{2} \beta^{2}}\right] \ln \left(1+e^{-\sqrt{k^{2} \beta^{2}+M^{2} \beta^{2}}}\right) \\
& +m \beta \frac{\sqrt{2}}{2 \pi} \sum_{l=0}^{S-2} \int_{-\infty}^{+\infty} d k\left[\frac{l+1}{m^{2} \beta^{2}(l+1)^{2} / 2+k^{2} \beta^{2}}\right] \ln \left(1+e^{-\sqrt{k^{2} \beta^{2}+M^{2} \beta^{2}}}\right) \\
& =-2 \frac{\ln 2}{\beta} \\
& +m \beta \frac{\sqrt{2}}{2 \pi} \sum_{l=0}^{S-1} 2 \int_{0}^{\infty} d k\left[\frac{l+1}{m^{2} \beta^{2}(l+1)^{2} / 2+k^{2} \beta^{2}}\right] \ln \left(1+e^{-\sqrt{k^{2} \beta^{2}+M^{2} \beta^{2}}}\right) \\
& +m \beta \frac{\sqrt{2}}{2 \pi} \sum_{l=0}^{S-2} 2 \int_{0}^{\infty} d k\left[\frac{l+1}{m^{2} \beta^{2}(l+1)^{2} / 2+k^{2} \beta^{2}}\right] \ln \left(1+e^{-\sqrt{k^{2} \beta^{2}+M^{2} \beta^{2}}}\right)
\end{aligned}
$$


Da mesma forma que ocorreu para o caso puramente bosônico, apenas os dois primeiros termos de 5.68 puderam ter a exponencial aproximada para $\beta \rightarrow 0$. Nos casos em que temos integral, pelo fato da variável $k$ ser integrada de 0 a $\infty$ não podemos aproximar a exponencial que contém os termos $k \beta$.

Façamos nas integrais as seguintes mudanças de variável:

$$
x=k \beta ; \quad d x=d k \beta ; \quad y_{1}^{2}=m^{2} \beta^{2} / 2 ; \quad y_{2}^{2}=M^{2} \beta^{2} .
$$

Temos:

$$
\begin{aligned}
\Gamma_{F}(T \rightarrow \infty) & =-2 \frac{\ln 2}{\beta} \\
& +m \frac{\sqrt{2}}{\pi} \sum_{l=0}^{S-1} \int_{0}^{\infty} d x\left[\frac{l+1}{(l+1)^{2} y_{1}^{2}+x^{2}}\right] \ln \left(1+e^{-\sqrt{x^{2}+y_{2}^{2}}}\right) \\
& +m \frac{\sqrt{2}}{\pi} \sum_{l=0}^{S-2} \int_{0}^{\infty} d x\left[\frac{l+1}{(l+1)^{2} y_{1}^{2}+x^{2}}\right] \ln \left(1+e^{-\sqrt{x^{2}+y_{2}^{2}}}\right)
\end{aligned}
$$

$\operatorname{Para} S=1$ :

$$
\begin{aligned}
\Gamma_{F}(T \rightarrow \infty) & =-2 \frac{\ln 2}{\beta}+m \frac{\sqrt{2}}{\pi} \underbrace{\int_{0}^{\infty} d x\left[\frac{1}{y_{1}^{2}+x^{2}}\right] \ln \left(1+e^{-\sqrt{x^{2}+y_{2}^{2}}}\right)}_{A_{1}} \\
& =-2 \frac{\ln 2}{\beta}+m C_{F 1}
\end{aligned}
$$

Para $S=2$ :

$$
\begin{aligned}
\Gamma_{F}(T \rightarrow \infty) & =-2 \frac{\ln 2}{\beta}+m \frac{2 \sqrt{2}}{\pi} \underbrace{\int_{0}^{\infty} d x\left[\frac{1}{y_{1}^{2}+x^{2}}\right] \ln \left(1+e^{-\sqrt{x^{2}+y_{2}^{2}}}\right)}_{A_{1}} \\
& +m \frac{2 \sqrt{2}}{\pi} \underbrace{\int_{0}^{\infty} d x\left[\frac{1}{4 y_{1}^{2}+x^{2}}\right] \ln \left(1+e^{-\sqrt{x^{2}+y_{2}^{2}}}\right)}_{A_{2}} \\
& =-2 \frac{\ln 2}{\beta}+m\left(C_{1}+C_{2}\right)=-2 \frac{\ln 2}{\beta}+m C_{F 2}
\end{aligned}
$$

Observa-se que, de maneira análoga ao que ocorre com a contribuição bosônica, a 
contribuição fermiônica, para ambos os valores de $S$, também é uma constante com dimensão de massa.

A correção da massa do sóliton, a temperatura finita, é dada por:

$$
M_{c}=M_{c l}+\Gamma_{B}(T)+\Gamma_{F}(T)=\frac{2 \sqrt{2} m^{3}}{3 \lambda}+\Gamma_{B}(T)+\Gamma_{F}(T)
$$

Para $S=1$, tem-se (Veja 4.58$)$ :

$$
M_{c}=\frac{2 \sqrt{2} m^{3}}{3 \lambda}-\frac{1}{\beta}[\ln (8 \sqrt{6})-\ln (m \beta)]-m\left(C_{B}-C_{F 1}\right)
$$

Para $S=2$, tem-se:

$$
M_{c}=\frac{2 \sqrt{2} m^{3}}{3 \lambda}-\frac{1}{\beta}[\ln (8 \sqrt{6})-\ln (m \beta)]-m\left(C_{B}-C_{F 2}\right)
$$




\section{Conclusões}

Neste trabalho foi feito um estudo sobre um modelo de sóliton a temperatura finita, mais precisamente o kink do modelo $\lambda \phi^{4}$, acoplado a um campo fermiônico. O objetivo foi calcular as correções à massa do sóliton devido à contribuição das flutuações quânticas (bosônicas e fermiônicas) em torno da solução clássica do kink, observando como estas se comportam a temperatura finita e a temperatura zero.

A temperatura zero, constatamos que a contribuição quântica ao sóliton aumenta o valor da sua massa para o caso $S=1\left(\lambda=2 g^{2}\right)$ e a diminui para $S=2\left(\lambda=g^{2} / 2\right)$. Veja 5.65 e 5.67, respectivamente. Observe que no primeiro caso a razão das massas das flutuações bosônicas e fermiônicas é: $M_{F} / M_{B}=1 / 2$ e no segundo $M_{F} / M_{B}=1$.

No trabalho [7] foram calculadas as contribuições das flutuações para o mesmo modelo, mas em quatro dimensões. Naquele caso, o sóliton é representado por uma parede e constatou-se que as flutuações (para ambos os valores de $\lambda$ ) diminuem a tensão superficial (massa sobre área).

A temperatura finita (para $T \rightarrow \infty$ ) observa-se que a contribuição das flutuações (bosônica e fermiônica) à massa clássica são constantes com dimensão de massa como esperado (Veja 5.74 e 5.75). O trabalho ainda está em andamento e não pudemos calcular as integrais que nos dariam o valor exato das constantes (Veja 4.60, 5.71 e 5.72), assim, não foi possível chegar a maiores conclusões. 



\section{A. Integrais funcionais}

\section{A.1. Integral funcional gaussiana para variáveis bosônicas}

Vamos relembrar o resultado da integral Gaussiana:

$$
\int_{-\infty}^{\infty} d x e^{-\alpha x^{2}}=\sqrt{\frac{\pi}{\alpha}}
$$

Agora consideremos uma integral funcional:

$$
\begin{aligned}
\int \mathcal{D} a e^{-\int d x \alpha a^{2}(x)} & \rightarrow \int \prod_{x} d a(x) e^{-\sum_{x} \alpha a^{2}(x)}=\int \prod_{x} d a(x) \prod_{x} e^{-\alpha a^{2}(x)} \\
& =\prod_{x} \int d a(x) e^{-\alpha a^{2}(x)}=\prod_{x} \sqrt{\frac{\pi}{\alpha}}
\end{aligned}
$$

Este mesmo resultado se aplica se tivermos uma função $f(x)$ ao invés da constante $\alpha:$

$$
\int \mathcal{D} a e^{-\int d x f(x) a^{2}(x)}=\prod_{x} \sqrt{\frac{\pi}{f(x)}} .
$$

Se pensarmos em $a(x)$ como um vetor dimensionalmente infinito, então podemos pensar em $f(x)$ como uma matriz diagonal infinita:

$$
f(x, y)=f(x) \delta(x-y)
$$


onde $f(x)$ representa os elementos da matriz diagonal, que correspondem, também, aos seus autovalores.

Sabemos que o produto dos autovalores de uma matriz é igual ao seu determinante [18], assim:

$$
\prod_{x} \sqrt{\frac{\pi}{f(x)}} \rightarrow(\sqrt{\pi})^{\infty} \prod_{x} \frac{1}{\sqrt{f(x)}}=\frac{(\sqrt{\pi})^{\infty}}{\sqrt{\operatorname{det} f}}=\operatorname{det}^{-\frac{1}{2}}\left(\frac{f}{\pi}\right)
$$

ou seja:

$$
\int \mathcal{D} a e^{-\int d x f(x) a^{2}(x)}=\operatorname{det}^{-\frac{1}{2}}\left(\frac{f}{\pi}\right)=C \operatorname{det}(f) .
$$

O número infinito que aparece no numerador é, geralmente, cancelado através de normalização, então não precisamos nos preocupar com seu valor exato.

Vamos pensar agora no caso geral, em que ao invés de uma matriz diagonal $f(x)$ possuimos um operador não diagonal, da seguinte forma:

$$
\int \mathcal{D} a e^{-\int d x f(x) a^{2}(x)} \rightarrow \int \mathcal{D} a e^{-a^{T} G a}
$$

Considerando que $G$ é uma matriz real e simétrica, pode ser diagonalizada de acordo com

$$
\tilde{a}=U a
$$

$$
\tilde{G}=U G U^{-1}=U G U T=\operatorname{diag}\left(g_{1} \ldots g_{i+n} \ldots\right) .
$$

Temos que $U U^{T}=I$, então:

$$
\tilde{a^{T}} \tilde{G} \tilde{a}=a^{T} U^{T} U G U^{T} U a=a^{T} G a .
$$


Ou seja, o integrando não muda para o caso geral. Assim:

$$
\int \mathcal{D} a e^{-a^{T} G a}=\prod_{i} \int d \tilde{a}_{i} e^{g_{i} \tilde{a}^{2}}=C \operatorname{det}(G)
$$

\section{A.2. Integral funcional gaussiana para variáveis fermiônicas}

Para estudo do campo fermiônico é indispensável a introdução de variáveis anticomutantes, que introduziremos brevemente a seguir. Sejam $\eta_{1}$ e $\eta_{2}$ duas variáveis de Grassmann:

$$
\left\{\eta_{1}, \eta_{2}\right\}=0
$$

ou seja, $\eta_{1}^{2}=0$. As regras de diferenciação para variáveis anticomutantes seguem [18]:

$$
\begin{gathered}
\frac{d}{d \eta_{i}} 1=0, \quad \frac{d}{d \eta_{i}} \eta_{j}=\delta_{i j} \\
\frac{d}{d \eta_{i}} \eta_{1} \eta_{2}=\delta_{i 1} \eta_{2}-\delta_{i 2} \eta_{1}
\end{gathered}
$$

ou seja, no caso geral:

$$
\frac{d}{d \eta_{j}} \eta_{i 1} \eta_{i 2} \ldots \eta_{i m}=\delta_{j i 1} \eta_{i 2} \ldots \eta_{i m}-\delta_{j i 2} \eta_{i 1} \eta_{i 3} \ldots \eta_{i m}+(-1)^{m-1} \delta_{j i m} \eta_{i 1} \eta_{i 2} \ldots \eta_{i_{m-1}}
$$

Para qualquer função $f(\eta)$, a sua expansão em série de potências deve ser da forma:

$$
f(\eta)=a+b \eta
$$


uma vez que $\eta^{2}=0$. Portanto, $d^{2} f / d \eta^{2}=0$, assim:

$$
\left\{\frac{d}{d \eta}, \frac{d}{d \eta}\right\}=0
$$

Também queremos integrar essas variáveis para poder trabalhar com elas no tratamento de férmions, para isso definiremos os resultados de duas integrais:

$$
\begin{gathered}
\int d \eta \equiv 0 \\
\int d \eta \eta \equiv 1 .
\end{gathered}
$$

Se estivermos trabalhando com mais de uma variável de Grassmann, temos:

$$
\int d \eta_{1} d \eta_{2} \eta_{2} \eta_{1}=1
$$

Como o campo de Dirac está definido no plano complexo, devemos definir as variáveis de Grassmann no espaço complexo também. Assim, temos:

$$
(\theta \eta)^{*} \equiv \eta^{*} \theta^{*}=-\theta^{*} \eta^{*}
$$

Podemos definir as variáveis de modo conveniente como:

$$
\eta=\frac{\xi_{1}+i \xi_{2}}{\sqrt{2}}, \quad \eta^{*}=\frac{\xi_{1}-i \xi_{2}}{\sqrt{2}}
$$

em que

$$
\begin{gathered}
\int d \eta \eta=1, \quad \int d \eta^{*} \eta^{*}=1, \\
\int d \eta=\int d \eta^{*}=\int d \eta \eta^{*}=\int d \eta^{*} \eta=0
\end{gathered}
$$


e

$$
\int d \eta^{*} d \eta\left(\eta \eta^{*}\right)=\int d \eta_{1} d \eta_{2} J\left(\frac{\partial\left(\eta^{*}, \eta\right)}{\partial\left(\eta_{2}, \eta_{1}\right)}\right)\left\{-i \frac{\left.\eta_{1} \eta_{2}\right)}{2}+i \frac{\eta_{2} \eta_{1}}{2}\right\}=-i \int d \eta_{1} d \eta_{2} i \eta_{2} \eta_{1}=1
$$

onde $J\left(\frac{\partial\left(\eta^{*}, \eta\right)}{\partial\left(\eta_{2}, \eta_{1}\right)}\right)$ é o determinante do jacobiano da transformação, dado por:

$$
J\left(\frac{\partial\left(\eta^{*}, \eta\right)}{\partial\left(\eta_{2}, \eta_{1}\right)}\right)=\left|\begin{array}{ll}
\frac{\partial \eta^{*}}{\partial \eta_{2}} & \frac{\partial \eta^{*}}{\partial \eta_{1}} \\
\frac{\partial \eta}{\partial \eta_{2}} & \frac{\partial \eta}{\partial \eta_{1}}
\end{array}\right|=-i
$$

Consideremos, primeiramente, a integral gaussiana unidimensional:

$$
\int d \eta^{*} d \eta e^{-\eta^{*} m \eta}=\int d \eta^{*} d \eta\left(1-\eta^{*} m \eta\right)=m
$$

Para o caso geral, n-dimensional, temos:

$$
\int \mathcal{D} \eta^{*} \mathcal{D} \eta f\left(\eta^{*}, \eta\right)=\int d \eta_{1}^{*} \ldots d \eta_{N}^{*} d \eta_{1} \ldots d \eta_{N} \exp \left(-\sum_{i, j=1}^{N} \eta_{i}^{*} m_{i j} \eta_{j}\right)
$$

Devido às propriedades das variáveis de Grassmann, observa-se que para $i \neq j$ o resultado da integral é zero. Assim, vamos diagonalizar a matriz $M$ e chamá-la de $M^{\prime}$. Com isso, teremos:

$$
\begin{aligned}
\int d \eta_{1}^{*} \ldots d \eta_{N}^{*} d \eta_{1} \ldots d \eta_{N} \prod_{i=1}^{N} \exp \left(\eta_{i}^{*} m_{i i}^{\prime} \eta_{i}\right) & =\int d \eta_{1}^{*} \ldots d \eta_{N}^{*} d \eta_{1} \ldots d \eta_{N} \prod_{i=1}^{N}\left(1-\eta_{i}^{*} m_{i i}^{\prime} \eta_{i}\right) \\
& =C \operatorname{det} M
\end{aligned}
$$

Se fizermos $N \rightarrow \infty$, teremos:

$$
\int \mathcal{D} \eta^{*} \mathcal{D} \eta e^{-\int d x \eta(x) g(x, y) \eta(y)}=C \operatorname{det} g
$$

onde $\mathcal{D} \eta^{*} \mathcal{D} \eta=\prod_{x}\left(d \eta^{*}(x) d \eta(x)\right)$. 



\section{B. Demonstração da identidade $\ln \operatorname{det} A=\operatorname{tr} \ln A$}

Seja uma matriz diagonal:

$$
B_{d}=\left[\begin{array}{rrr}
b_{1} & \cdots & 0 \\
\vdots & \ddots & \vdots \\
0 & \cdots & b_{n}
\end{array}\right]
$$

e $A_{d}$ definido como:

$$
A_{d}=e^{B_{d}}=I+B_{d}+\frac{1}{2} B_{d} B_{d}+\ldots
$$

ou seja, $B_{d}=\ln A_{d}$. Se elevamos $B_{d}$ a um número qualquer $m$, temos:

$$
B_{d}{ }^{m}=\left[\begin{array}{rrr}
b_{1}{ }^{m} & \cdots & 0 \\
\vdots & \ddots & \vdots \\
0 & \cdots & b_{n}{ }^{m}
\end{array}\right]
$$

Desta forma:

$$
A_{d}=I+B_{d}+\frac{1}{2} B_{d} B_{d}+\ldots=\left[\begin{array}{rrr}
1+b_{1}+\frac{1}{2} b_{1}{ }^{2}+\ldots & \ldots & 0 \\
\vdots & \ddots & \vdots \\
0 & \ldots & 1+b_{n}+\frac{1}{2} b_{n}{ }^{2}+\ldots
\end{array}\right]
$$

que podemos também escrever como:

$$
A_{d}=\left[\begin{array}{rrr}
e^{b_{1}} & \cdots & 0 \\
\vdots & \ddots & \vdots \\
0 & \cdots & e^{b_{n}}
\end{array}\right]
$$


Os elementos da matriz diagonal $A_{d}$ são iguais a $a_{i}=e^{b_{i}}$.

Consideremos agora uma matriz unitária inversível $U$, tal que $U U^{-1}=I$ e $\operatorname{det} U=1$.

Se multiplicarmos a equação B.1 à esquerda por $U$ e à direita por $U^{-1}$, teremos:

$$
\begin{aligned}
U A_{d} U^{-1} & =U I U^{-1}+U B_{d} U^{-1}+\frac{1}{2} U B_{d} B_{d} U^{-1}+\ldots \\
& =I+U B_{d} U^{-1}+\frac{1}{2} U B_{d} U^{-1} U B_{d} U^{-1}+\ldots
\end{aligned}
$$

Vamos definir $A \equiv U A_{d} U^{-1}$ e $B=U B_{d} U^{-1}$. Desta forma $A=e^{B}$, que pode ser escrito como $B \equiv \ln A$. Com isso, temos:

$$
\ln (\operatorname{det}(A))=\ln \left(\operatorname{det}\left(U A_{d} U^{-1}\right)\right)=\ln \left(\operatorname{det}(U) \operatorname{det}\left(A_{d}\right) \operatorname{det}\left(U^{-1}\right)\right)=\ln \left(\operatorname{det}\left(A_{d}\right)\right) .
$$

Como $A_{d}$ é uma matriz diagonal, o seu determinante é igual ao produto de seus autovalores, assim:

$\ln \left(\operatorname{det}\left(A_{d}\right)\right)=\ln \left(\prod_{i=1}^{n} a_{i}\right)=\ln \left(\prod_{i=1}^{n} e^{b_{i}}\right)=\ln \left(\exp \left(\sum_{i=1}^{n} b_{i}\right)\right)=\sum i=1^{n} b_{i}=\operatorname{tr}\left(B_{d}\right)$

Mas se $B=U B_{d} U^{-1}$, então $B_{d}=U^{-1} B U$ e, portanto:

$$
\ln (\operatorname{det}(A))=\ln \left(\operatorname{det}\left(A_{d}\right)\right)=\operatorname{tr}\left(B_{d}\right)=\operatorname{tr}\left(U^{-1} B U\right)=U_{i j}{ }^{-1} B_{j k} U_{k i} .
$$

Como são elementos de matrizes posso colocar na ordem que for mais conveniente, assim:

$$
\ln (\operatorname{det}(A))=B_{j k} U_{k i} U_{i j}{ }^{-1}=B_{j k} \delta_{k j}=B_{i i} .
$$

Índices repetidos indicam somatória sobre esses índices, portanto, combinando B.6 com a definição de $B \equiv \ln A$, teremos: 


$$
B_{i i}=\operatorname{tr}(B)=\operatorname{tr}(\ln A),
$$

ou seja:

$$
\ln (\operatorname{det}(A))=\operatorname{tr}(\ln A),
$$

como queríamos demonstrar. 



\section{Alguns resultados importantes}

C.1. Cálculo de $\sum_{n=-\infty}^{\infty} \ln \left(\alpha^{2}+\frac{4 \pi^{2} n^{2}}{\beta^{2}}\right)$

Nesta seção faremos a demonstração da identidade

$$
\sum_{n=-\infty}^{\infty} \ln \left(\alpha^{2}+\frac{4 \pi^{2} n^{2}}{\beta^{2}}\right)=\alpha \beta+2 \ln \left(1-e^{-\alpha \beta}\right)+\mathcal{C}
$$

Temos que:

$$
\ln \left(\alpha^{2}+\frac{4 \pi^{2} n^{2}}{\beta^{2}}\right)=\ln \left(\alpha^{2} \beta^{2}+4 \pi^{2} n^{2}\right)-\ln \beta^{2}
$$

onde $\mathcal{C}$ é uma constante independente de $\alpha$. Vamos nos preocupar apenas com o termo dependente de $\alpha$ e $n$. Tem-se [15]:

$$
\frac{d}{d x^{2}}\left[\sum_{n=-\infty}^{\infty} \ln \left(x^{2}+b^{2} n^{2}\right)\right]=\sum_{n=-\infty}^{\infty} \frac{1}{x^{2}+b^{2} n^{2}}=\frac{\pi}{x b} \operatorname{coth}(\pi x / b)
$$

onde $b^{2}=4 \pi^{2}$ e $x^{2}=\alpha^{2} \beta^{2}$. Então:

$$
\int_{0}^{\alpha^{2} \beta^{2}} d x^{2} \frac{d}{d x^{2}}\left[\sum_{n=-\infty}^{\infty} \ln \left(x^{2}+b^{2} n^{2}\right)\right]=\underbrace{\int_{0}^{\alpha^{2} \beta^{2}} d x^{2}\left[\frac{\pi}{x b} \operatorname{coth}(\pi x / b)\right]}_{I}
$$

Após mudança de variável, tem-se: 


$$
\begin{aligned}
I & =\int_{0}^{\alpha^{2} \beta^{2}} d u \frac{\pi}{b \sqrt{u}} \operatorname{coth}(\pi \sqrt{u} / b)=\frac{2 \pi}{b} \int_{0}^{\alpha \beta} d y \operatorname{coth}(\pi y / b)=\left.\frac{2 \pi}{b} \frac{\ln \sinh (\pi y / b)}{\pi / b}\right|_{0} ^{\alpha \beta} \\
& =2 \ln \sinh (\alpha \beta / 2)=2 \ln \left[\frac{e^{\alpha \beta / 2}-e^{-\alpha \beta / 2}}{2}\right]=\alpha \beta+2 \ln \left(1-e^{-\alpha \beta}\right)-2 \ln 2
\end{aligned}
$$

E portanto:

$$
\begin{aligned}
\sum_{n=-\infty}^{\infty} \ln \left(\alpha^{2}+\frac{4 \pi^{2} n^{2}}{\beta^{2}}\right) & =\alpha \beta+2 \ln \left(1-e^{-\alpha \beta}\right)-2 \ln 2-\ln \beta^{2} \\
& =\alpha \beta+2 \ln \left(1-e^{-\alpha \beta}\right)+\mathcal{C}
\end{aligned}
$$

C.2. Cálculo de $\sum_{n=-\infty}^{\infty} \ln \left(\alpha^{2}+\frac{(2 n+1)^{2} \pi^{2}}{\beta^{2}}\right)$

Analogamente ao que foi feito na seção anterior, demonstraremos a seguinte identidade:

$$
\sum_{n=-\infty}^{\infty} \ln \left(\alpha^{2}+\frac{(2 n+1)^{2} \pi^{2}}{\beta^{2}}\right)=\alpha \beta+2 \ln \left(1+e^{-\alpha \beta}\right)+\mathcal{C}
$$

Temos que:

$$
\begin{aligned}
\ln \left(\frac{(2 n+1)^{2} \pi^{2}}{\beta^{2}}+\alpha^{2}\right) & =\ln \left((2 n+1)^{2} \pi^{2}+\alpha^{2} \beta^{2}\right)-\ln \beta^{2} \\
& =\ln \left((2 n+1)^{2} \pi^{2}+x^{2}\right)-\ln \beta^{2}
\end{aligned}
$$

Levando em consideração apenas o termo dependente de $n$, tem-se:

$$
\frac{d}{d x^{2}} \ln \operatorname{det} \hat{A}=\sum_{n} \frac{1}{x^{2}+(2 n+1)^{2} \pi^{2}}=\frac{1}{2 x} \tanh (x / 2)
$$

Então:

$$
\int d x^{2} \frac{d}{d x^{2}} \ln \operatorname{det} \hat{A}=\int d x^{2} \frac{1}{2 x} \tanh (x / 2)
$$

Após mudança de variável: 


$$
\begin{aligned}
I & =\int_{0}^{\alpha^{2} \beta^{2}} d x^{2} \frac{1}{2 x} \tanh (x / 2)=\int_{0}^{\alpha \beta} d y \tanh (y / 2)=\left.2 \ln \cosh (x / 2)\right|_{0} ^{\alpha \beta} \\
& =\alpha \beta+2 \ln \left(1+e^{-\alpha \beta}\right)-2 \ln 2
\end{aligned}
$$

Portanto:

$$
\begin{aligned}
\sum_{n=-\infty}^{\infty} \ln \left(\frac{(2 n+1)^{2} \pi^{2}}{\beta^{2}}+\alpha^{2}\right) & =\alpha \beta+2 \ln \left(1+e^{-\alpha \beta}\right)-2 \ln 2-\ln \beta^{2} \\
& =\alpha \beta+2 \ln \left(1+e^{-\alpha \beta}\right)+\mathcal{C}
\end{aligned}
$$

\section{C.3. Modo zero}

Da fórmula C.1 podemos escrever:

$$
\begin{aligned}
\sum_{n \neq 0} \ln \left(\alpha^{2}+\omega_{n}^{2}\right) & =\sum_{n \neq 0} \ln \left(\alpha^{2}+\frac{4 \pi^{2} n^{2}}{\beta^{2}}\right) \\
& =\alpha \beta+2 \ln \left(1-e^{-\alpha \beta}\right)-2 \ln 2-\ln \beta^{2}-\left(\ln \left(\alpha^{2} \beta^{2}\right)-\ln \beta^{2}\right) \\
& =\alpha \beta+2 \ln \left(1-e^{-\alpha \beta}\right)-2 \ln 2-2 \ln \alpha \beta=\alpha \beta-2 \ln 2+2 \ln \left(\frac{1-e^{-\alpha \beta}}{\alpha \beta}\right)
\end{aligned}
$$

Para $\alpha \rightarrow 0$ :

$$
\sum_{n \neq 0} \ln \left(\alpha^{2}+\frac{4 \pi^{2} n^{2}}{\beta^{2}}\right) \rightarrow-2 \ln 2
$$

\section{C.4. Integral divergente}

Utilizando cut-off, vamos determinar o valor da seguinte integral divergente:

$$
I=\int_{-\infty}^{+\infty} d k \frac{\sqrt{k^{2}+b}}{k^{2}+a}
$$

Vamos substituir os limites por uma variável $\Lambda$ : 


$$
\begin{aligned}
I & =\int_{-\Lambda}^{\Lambda} d k \frac{\sqrt{k^{2}+b}}{k^{2}+a}=2 \int_{0}^{\Lambda} d k \frac{\sqrt{k^{2}+b}}{k^{2}+a} \\
& =\frac{\sqrt{b-a}}{\sqrt{a}} \arctan \left(\frac{\Lambda \sqrt{b-a}}{\sqrt{a\left(b+\Lambda^{2}\right)}}\right)+\ln \left(\sqrt{b+\Lambda^{2}}+\Lambda\right)-\ln \sqrt{b} \\
& =\frac{\sqrt{b-a}}{\sqrt{a}} \arctan \left(\sqrt{\frac{b-a}{a}}\left(1-\frac{b}{2 \Lambda^{2}}\right)\right)+\ln \Lambda+\ln \left(2+b / 2 \Lambda^{2}\right)-\ln \sqrt{b}
\end{aligned}
$$

Fazendo $\Lambda \rightarrow \infty$ :

$$
I=\frac{\sqrt{b-a}}{\sqrt{a}} \arctan \left(\sqrt{\frac{b-a}{a}}\right)+\ln \Lambda+\ln 2-\ln \sqrt{b}
$$




\section{Conjugação de carga}

Consideremos a equação de autovalores em duas dimensões:

$$
\begin{gathered}
\left(\gamma_{E}^{\mu} \partial_{\mu}+\phi(x)\right) \chi(x, t)=\varepsilon \chi(x, t) \\
\Rightarrow\left(\gamma_{E}^{0} \partial_{0}+\gamma_{E}^{1} \partial_{x}+\phi(x)\right) \chi(x, t)=\varepsilon \chi(x, t)
\end{gathered}
$$

Fazendo a mudança de variável $(x, t) \rightarrow(-x, t)$, tem-se:

$$
\left(\gamma_{E}^{0} \partial_{0}-\gamma_{E}^{1} \partial_{x}+\phi(-x)\right) \chi(-x, t)=\varepsilon \chi(-x, t)
$$

Multiplicando esta equação pela matriz quadrada $B$ (pela esquerda), teremos:

$$
\left(B \gamma_{E}^{0} \partial_{0}-B \gamma_{E}^{1} \partial_{x}+\phi(-x) B\right) \chi(-x, t)=\varepsilon B \chi(-x, t)
$$

que também podemos escrever como

$$
\left(B \gamma^{0} B^{-1} \partial_{0}-B \gamma^{1} B^{-1} \partial_{x}+\phi(-x)\right) B \chi(-x, t)=\varepsilon B \chi(-x, t)
$$

Se escolhermos $B=\gamma^{0}$, temos:

$$
B^{-1}=-\gamma^{0}
$$

$$
B \gamma^{0} B^{-1}=\gamma^{0}
$$




$$
B \gamma^{1} B^{-1}=-\gamma^{1}
$$

e, portanto:

$$
\left(\gamma^{0} \partial_{0}+\gamma^{1} \partial_{x}+\phi(-x)\right)\left(\gamma^{0} \chi(-x, t)\right)=\varepsilon\left(\gamma^{0} \chi(-x, t)\right)
$$

Como para o sóliton temos que $\phi(-x)=-\phi(x)$, obtem-se:

$$
\left(\gamma^{\mu} \partial_{\mu}-\phi(x)\right)\left(\gamma^{0} \chi(-x, t)\right)=\varepsilon\left(\gamma^{0} \chi(-x, t)\right)
$$

Portanto, para cada autofunção $\chi(x, t)$ do operador $O_{+}=\not \partial+\phi(x)$ com autovalor $\varepsilon$, tem-se uma autofunção $\gamma^{0} \chi(-x, t)$ com o mesmo autovalor $\varepsilon$ para o operador $O_{-}=$ $\not \partial-\phi(x)$. Desta forma:

$$
\operatorname{det}(\not \partial+\phi(x))=\prod(\varepsilon)=\operatorname{det}(\not \partial-\phi(x)),
$$

e portanto:

$$
\operatorname{det}(\not \partial+\phi(x))=\operatorname{det}^{1 / 2}(\not \partial+\phi(x)) \operatorname{det}^{1 / 2}(\not \partial-\phi(x)) .
$$




\section{E. Propagadores de Feynman bosônico e fermiônico}

\section{E.1. Propagador bosônico}

O gerador funcional de um sistema no espaço euclidiano é dado por:

$$
Z[J]=N \int \mathcal{D} \phi e^{-S_{E}}=N \int \mathcal{D} \phi e^{-\int d^{4} x\left[\mathcal{L}_{E}(\phi)-J(x) \phi(x)\right]} .
$$

Para uma lagrangiana da forma:

$$
\mathcal{L}_{E}=\frac{1}{2} \partial_{\mu} \phi \partial_{\mu} \phi+\frac{1}{2} m^{2} \phi^{2}=\frac{1}{2} \phi\left(-\square+m^{2}\right) \phi=\frac{1}{2} \phi \hat{O}_{E} \phi
$$

temos:

$S_{E}[\phi, J]=\int d^{4} x\left[\frac{1}{2} \phi\left(-\square+m^{2}\right) \phi-J(x) \phi(x)\right]=\int d^{4} x\left[\frac{1}{2} \phi \hat{O}_{E} \phi-J(x) \phi(x)\right]$.

Façamos a seguinte expansão no campo:

$$
\phi=\tilde{\phi}+\phi_{0}
$$

Escolhendo $\phi_{0}$ de tal forma que:

$$
\hat{O}_{E} \phi_{0}(x)=J(x) .
$$

Teremos então: 


$$
\begin{aligned}
S_{E}[\phi, J] & =\int d^{4} x\left(\frac{1}{2} \phi(x) \hat{O}_{E} \phi(x)-J(x) \phi(x)\right) \\
& =\int d^{4} x\left(\frac{1}{2} \tilde{\phi} \hat{O}_{E} \tilde{\phi}+\tilde{\phi} \hat{O}_{E} \phi_{0}+\frac{1}{2} \phi_{0} \hat{O}_{E} \phi_{0}-J \tilde{\phi}-J \phi_{0}\right) \\
& =\frac{1}{2} \tilde{\phi} \hat{O}_{E} \tilde{\phi}-\frac{1}{2} J \phi_{0} .
\end{aligned}
$$

A solução para E.4 é dada por [3]:

$$
\phi_{0}(x)=\alpha \int \Delta_{F}(x-y) J(y) d^{4} y
$$

Assim:

$$
\alpha \hat{O}_{E} \Delta_{F}(x-y)=\delta^{4}(x-y)
$$

Temos que:

$$
\begin{aligned}
Z[J] & =e^{-W(J)}=\int \mathcal{D} \phi e^{\int d^{4} x\left[-\frac{1}{2} \phi(x) \hat{O}_{E} \phi(x)+J(x) \phi(x)\right]} \\
& =\int \mathcal{D} \tilde{\phi} e^{\int d^{4} x\left[-\frac{1}{2} \tilde{\phi}(x) \hat{O}_{E} \tilde{\phi}(x)\right]} e^{\int d^{4} x d^{4} y\left[\frac{\alpha}{2} J(x) \Delta_{F}(x-y) J(y)\right]}
\end{aligned}
$$

onde o gerador das funções de Green conexas é dado por:

$$
\begin{aligned}
W(J) & =-\ln \int \mathcal{D} \phi e^{\int d^{4} x\left[-\frac{1}{2} \phi(x) \hat{O}_{E} \phi(x)+J(x) \phi(x)\right]} \\
& =-\frac{\alpha}{2} \int d^{4} x d^{4} y J(x) \Delta_{F}(x-y) J(y) .
\end{aligned}
$$

As funções de Green são dadas diferenciando-se este operador com respeito a $J$ da seguinte forma:

$$
\begin{aligned}
\frac{\delta W(J)}{\delta J(x)} & =-\frac{1}{Z} \int \mathcal{D} \phi \phi(x) e^{\int d^{4} x\left[-\frac{1}{2} \phi(x) \hat{O}_{E} \phi(x)+J(x) \phi(x)\right]} \\
& =-\alpha \int d^{4} y \Delta_{F}(x-y) J(y) \\
\frac{\delta^{2} W(J)}{\delta J(x) \delta J(y)} & =-\frac{1}{Z} \int \mathcal{D} \phi \phi(x) \phi(y) e^{\int d^{4} x\left[-\frac{1}{2} \phi(x) \hat{O}_{E} \phi(x)+J(x) \phi(x)\right]} \\
& =-\langle 0|T \phi(x) \phi(y)| 0\rangle=-\alpha \Delta_{F}(x-y) .
\end{aligned}
$$

Se escolhermos $\alpha=1$, teremos: 


$$
\Delta_{F}(x-y)=\langle 0|T \phi(x) \phi(y)| 0\rangle .
$$

Portanto, E.7 se torna:

$$
\hat{O}_{E} \Delta_{F}(x-y)=\delta^{4}(x-y) .
$$

Para $\hat{O}_{E}=\left(-\square+m^{2}\right)$ e a transformada de Fourier do propagador dada por:

$$
\Delta_{F}(x-y)=\int \frac{d^{4} p}{(2 \pi)^{4}} e^{-i p(x-y)} \Delta_{F}(p)
$$

teremos que o propagador da partícula livre no espaço euclidiano é dado por:

$$
\Delta_{F}(p)=\frac{1}{p^{2}+m^{2}}
$$

\section{E.2. Propagador fermiônico}

O gerador funcional de um sistema fermiônico no espaço euclidiano, em duas dimensões, é dado por:

$$
Z[J]=N \int \mathcal{D} \Psi \mathcal{D} \bar{\Psi} e^{-S_{E}}=N \int \mathcal{D} \Psi \mathcal{D} \bar{\Psi} e^{-\int d^{2} x\left[\mathcal{L}_{E}(\phi)+\bar{\rho}(x) \Psi(x)+\bar{\Psi} \rho(x)\right]}
$$

Onde $\rho$ e $\bar{\rho}$ são fontes externas e também variáveis de Grassmann.

Para uma lagrangiana da forma:

$$
\mathcal{L}_{E}=\bar{\Psi}(-\not \partial-i M) \Psi=\bar{\Psi} \hat{O}_{F} \Psi
$$

temos:

$$
S_{E}=\int d^{2} x\left[\bar{\Psi} \hat{O}_{F} \Psi+\bar{\rho}(x) \Psi(x)+\bar{\Psi} \rho(x)\right] .
$$

Façamos as seguintes expansões nos campos: 


$$
\begin{aligned}
& \Psi(x)=\Psi^{\prime}(x)+\alpha \int d^{2} y S_{x y} \rho(y) \\
& \bar{\Psi}(x)=\bar{\Psi}^{\prime}(x)+\alpha \int d^{2} y \bar{\rho}(y) \bar{S}_{y x},
\end{aligned}
$$

onde $\alpha$ é uma constante a ser determinada.

Teremos então:

$$
\begin{aligned}
S_{E}= & \int d^{2} x \bar{\Psi}^{\prime}(x) \hat{O}_{F} \Psi^{\prime}(x)+\alpha \iint d^{2} x d^{2} y \bar{\Psi}^{\prime}(x) \hat{O}_{F} S_{x y} \rho(y) \\
& +\alpha \iint d^{2} x d^{2} y \bar{\rho}(y) \bar{S}_{y x} \hat{O}_{F} \Psi^{\prime}(x)+\alpha^{2} \iiint d^{2} x d^{2} y d^{2} z \bar{\rho}(y) \bar{S}_{y x} \hat{O}_{F} S_{x z} \rho(z) \\
& +\int d^{2} x \bar{\rho}(x) \Psi^{\prime}(x)+\alpha \iint d^{2} x d^{2} y \bar{\rho}(x) S_{x y} \rho(y) \\
& +\int d^{2} x \bar{\Psi}^{\prime}(x) \rho(x)+\alpha \iint d^{2} x d^{2} y \bar{\rho}(y) \bar{S}_{y x} \rho(x)
\end{aligned}
$$

Os termos mistos se cancelam da seguinte forma:

$$
\alpha \iint d^{2} x d^{2} y \bar{\Psi}^{\prime}(x) \hat{O}_{F} S_{x y} \rho(y)+\iint d^{2} x d^{2} y \bar{\Psi}^{\prime}(x) \rho(y) \delta^{2}(x-y)=0 .
$$

Assim, chegamos à seguinte relação:

$$
\alpha \hat{O}_{F} S_{x y}+\delta_{x y}=0 ; \quad \hat{O}_{F} S_{x y}=-\frac{1}{\alpha} \delta_{x y}
$$

A seguir determinaremos o valor de $\alpha$ partindo do gerador funcional $Z$.

Dos vários cancelamentos que ocorrem na expansão em E.20, sobram apenas os seguintes termos:

$$
S_{E}=\int d^{2} x \bar{\Psi}^{\prime}(x) \hat{O}_{F} \Psi^{\prime}(x)+\alpha \iint d^{2} x d^{2} y \bar{\rho}(x) S_{x y} \rho(y) .
$$

Então: 


$$
\begin{aligned}
Z & =N \int \mathcal{D} \Psi \mathcal{D} \bar{\Psi} \exp \left\{-\int d^{2} x\left[\bar{\Psi}_{F} \Psi+\bar{\rho}(x) \Psi(x)+\bar{\Psi} \rho(x)\right]\right\} \\
& =N^{\prime} \underbrace{\int \mathcal{D} \Psi^{\prime} \mathcal{D} \bar{\Psi}^{\prime} \exp \left[-\int d^{2} x \bar{\Psi}^{\prime}(x) \hat{O}_{F} \Psi^{\prime}(x)\right]}_{C} \exp \left\{-\alpha \iint d^{2} x d^{2} y \bar{\rho}(x) S_{x y} \rho(y)\right\} \\
& =N^{\prime} \exp \left\{-\alpha \iint d^{2} x d^{2} y \bar{\rho}(x) S_{x y} \rho(y)\right\}
\end{aligned}
$$

onde $\mathrm{C}$ representa uma constante, resultado de uma integral que depende apenas de $\Psi$ e $\bar{\Psi}$.

As funções de Green são dadas diferenciando-se este operador com respeito às fontes externas $\rho$ e $\bar{\rho}[10]$. Vamos fazer para ambas as igualdades de Z. Obtemos:

$$
\begin{aligned}
\frac{\delta Z}{\delta \rho(y) \delta \bar{\rho}(x)} & =\int \mathcal{D} \Psi \mathcal{D} \bar{\Psi}(-\Psi(x)) \bar{\Psi}(y) \exp \left\{-\int d^{2} x\left[\bar{\Psi} \hat{O}_{F} \Psi+\bar{\rho}(x) \Psi(x)+\bar{\Psi} \rho(x)\right]\right\} \\
& =-\alpha S_{x y}
\end{aligned}
$$

Então, temos que a função de 2 pontos é dada por:

$$
\langle 0|T \Psi(x) \bar{\Psi}(y)| 0\rangle=\alpha S_{x y} \text {, ou seja, } \alpha=1 \text {. }
$$

Voltando a E.22, tem-se a seguinte igualdade:

$$
\hat{O}_{F} S_{x y}=-\delta_{x y}
$$

Através deste resultado podemos determinar o propagador fermiônico.

Para $\hat{O}_{F}=(-\not \partial-i M)$ e a transfomada de Fourier do propagador dada por:

$$
S_{F}(x-y)=\int \frac{d^{2} p}{(2 \pi)^{2}} e^{-i p(x-y)} S_{F}(p)
$$

teremos que o propagador da partícula livre no espaço euclidiano é:

$$
S_{F}(p)=-i \frac{\gamma^{\nu} p_{\nu}+M}{p^{2}+M^{2}}
$$





\section{F. Renormalização}

\section{F.1. Campo bosônico}

Conforme foi visto na seção 4.3, a lagrangiana renormalizada, após expansão do campo em $\phi=\phi_{c}+\Phi$, é dada por:

$$
\begin{aligned}
\mathcal{L}_{R} & =\hbar^{0}\left(-\frac{1}{2} \phi_{c} \partial_{\mu}^{2} \phi_{c}-\frac{1}{2} m^{2} \phi^{2}+\frac{1}{4} \lambda \phi_{c}^{4}+\frac{m^{4}}{4 \lambda}\right) \\
& +\hbar^{1 / 2}\left(-\phi_{c} \partial_{\mu}^{2} \Phi-m^{2} \phi_{c} \Phi+\lambda \phi_{c}^{3} \Phi\right) \\
& +\hbar\left(-\frac{1}{2} \Phi \partial_{\mu}^{2} \Phi-\frac{1}{2} m^{2} \Phi^{2}+\frac{3}{2} \lambda \phi_{c}^{2} \Phi^{2}-\frac{A}{2} \phi_{c} \partial_{\mu}^{2} \phi_{c}+\frac{B}{2} \phi_{c}^{2}+\frac{C}{4} \phi_{c}^{4}+H\right) \\
& +\hbar^{3 / 2}\left(\lambda \phi_{c} \Phi^{3}-A \phi_{c} \partial_{\mu}^{2} \Phi+B \phi_{c} \Phi+C \phi_{c}^{3} \Phi\right) \\
& +\hbar^{2}\left(\frac{\lambda}{4} \Phi^{4}-\frac{A}{2} \Phi \partial_{\mu}^{2} \Phi+\frac{B}{2} \Phi^{2}+\frac{3}{2} C \phi_{c}^{2} \Phi^{2}\right)
\end{aligned}
$$

onde $\phi_{c}=m / \sqrt{\lambda}$ é o campo clássico.

O propagador do bóson é (Veja E.15):

$$
\Delta_{F}(p)=\frac{1}{p^{2}+m^{2}}
$$

A função de 1 ponto, é dada por (Veja [19] e [20]): 


$$
\begin{aligned}
\left\langle\eta_{x}\right\rangle & =\left\langle 0\left|T \eta_{x} e^{-\frac{1}{\hbar} \int \mathcal{L}_{i n t} d^{2} z}\right| 0\right\rangle \\
& =\underbrace{\left\langle 0\left|T \eta_{x}\right| 0\right\rangle}_{(0)}+\underbrace{\left\langle 0\left|T \eta_{x}\left(-\frac{1}{\hbar}\right) \int \mathcal{L}_{i n t} d^{2} z\right| 0\right\rangle}_{(1)} \\
& +\underbrace{\left\langle 0\left|T \eta_{x}\left(-\frac{1}{\hbar}\right)^{2} \frac{1}{2} \int \mathcal{L}_{\text {int }}\left(z_{1}\right) \mathcal{L}_{\text {int }}\left(z_{2}\right) d^{2} z_{1} d^{2} z_{2}\right| 0\right\rangle}_{(2)},
\end{aligned}
$$

onde $T$ é o produto ordenado e $\mathcal{L}_{\text {int }}$ corresponde à lagrangiana de interação.

Primeiramente, vamos trabalhar com o termo de primeira ordem, (1). Para obter uma aproximação de ordem $\hbar^{1 / 2}$ precisamos de termos de $\mathcal{L}_{\text {int }}$ proporcionais a $\hbar^{3 / 2}$. Voltando a F.1, temos que:

$$
\mathcal{L}_{\text {int }}=\hbar^{3 / 2}\left(-A \phi_{c} \partial_{\mu}^{2} \Phi+B \phi_{c} \Phi+C \phi_{c}^{3} \Phi+\lambda \phi_{c} \Phi^{3}\right)
$$

Substituindo no termo (1) de F.3, obtem-se:

$$
\begin{aligned}
(1) & =\left\langle 0\left|T \eta_{x}(-\hbar)^{1 / 2} \int d^{2} z\left(-A \phi_{c} \partial_{z}^{2} \eta_{z}+B \phi_{c} \eta_{z}+C \phi_{c}^{3} \eta_{z}+\lambda \phi_{c} \eta_{z}^{3}\right)\right| 0\right\rangle \\
& =-\hbar^{1 / 2}[\underbrace{\int d^{2} z\left(-A \phi_{c}\right) \partial_{z}^{2} \Delta_{z x}}_{(1.1)}+\underbrace{\int d^{2} z \Delta_{z x}\left(B \phi_{c}+C \phi_{c}^{3}\right)}_{(1.2)}+\underbrace{\int d^{2} z \Delta_{z x}\left(3 \lambda \phi_{c} \Delta_{z z}\right)}_{(1.3)}] .
\end{aligned}
$$

Onde $^{1}$

$$
\begin{gathered}
(1.1)=0 \\
(1.2)=-\hbar^{1 / 2}\left(B \phi_{c}+C \phi_{c}^{3}\right) \int d^{2} z \Delta_{z x} \\
(1.3)=\int d^{2} z \Delta_{z x}\left(-\hbar^{1 / 2} 3 \lambda \phi_{c} \Delta_{z z}\right) \\
=\int d^{2} z \Delta_{z x}\left(-\hbar^{1 / 2} 3 \lambda \phi_{c} \int \frac{d^{2} k}{(2 \pi)^{2}} \frac{1}{\left(k^{2}+2 m^{2}\right)}\right)
\end{gathered}
$$

\footnotetext{
${ }^{1}$ Observe que no último termo usamos a transformada de fourier do propagador do bóson F.2
} 
De F.7 e F.8, temos que os gráficos de Feynman conexos e 1PI que contribuem para $\Gamma_{x}^{(1)}$ são dados por:

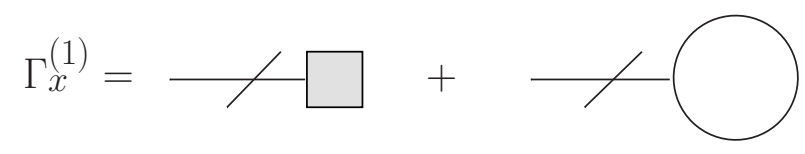

Sendo $\Gamma_{x}^{(1)}($ para $\hbar=1)$ igual a:

$$
\begin{aligned}
\Gamma_{x}^{(1)} & =-\left(B \phi_{c}+C \phi_{c}^{3}\right)-3 \lambda \phi_{c} \int \frac{d^{2} k}{(2 \pi)^{2}} \frac{1}{\left(k^{2}+2 m^{2}\right)} \\
& =-\frac{m}{\sqrt{\lambda}}\left[3 \lambda \int \frac{d^{2} k}{(2 \pi)^{2}} \frac{1}{\left(k^{2}+2 m^{2}\right)}+B+\frac{m^{2}}{\lambda} C\right]
\end{aligned}
$$

Façamos agora a aproximação com a função de 2 pontos, que é definida como [19]:

$$
\begin{aligned}
\left\langle 0\left|T \eta_{x} \eta_{y}\right| 0\right\rangle & =\left\langle 0\left|T \eta_{x} \eta_{y} e^{-\frac{1}{\hbar} \int \mathcal{L}_{i n t} d^{2} z}\right| 0\right\rangle \\
& =\underbrace{\left\langle 0\left|T \eta_{x} \eta_{y}\right| 0\right\rangle}_{(0)}-\underbrace{\frac{1}{\hbar} \int d^{2} z\left\langle 0\left|T \eta_{x} \eta_{y} \mathcal{L}_{\text {int }}(z)\right| 0\right\rangle}_{(1)} \\
& +\underbrace{\frac{1}{2 \hbar^{2}} \int d^{2} z_{1} d^{2} z_{2}\left\langle 0\left|T \eta_{x} \eta_{y} \mathcal{L}_{\text {int }}\left(z_{1}\right) \mathcal{L}_{\text {int }}\left(z_{2}\right)\right| 0\right\rangle}
\end{aligned}
$$

Vamos trabalhar com o termo de primeira ordem (1). Para obter uma aproximação de ordem $\hbar$ preciso dos termos de $\mathcal{L}_{\text {int }}$ proporcionais a $\hbar^{2}$. Novamente, de F.1, temos neste caso:

$$
\mathcal{L}_{\text {int }}=\hbar^{2}\left(-\frac{A}{2} \Phi \square \Phi+\frac{B}{2} \Phi^{2}+\frac{\lambda}{4} \Phi^{4}+\frac{3}{2} C \phi_{c}^{2} \Phi^{2}\right)
$$

Substituindo no termo de primeira ordem, (1), de F.10: 
$(1)=-\frac{1}{\hbar} \int d^{2} z\left\langle 0\left|T \eta_{x} \eta_{y} \mathcal{L}_{\text {int }}(z)\right| 0\right\rangle$

$$
\begin{aligned}
& =-\hbar \int d^{2} z\left\langle 0\left|T \eta_{x} \eta_{y}\left(-\frac{A}{2} \eta_{z} \square \eta_{z}+\frac{B}{2} \eta_{z}^{2}+\frac{\lambda}{4} \eta_{z}^{4}+\frac{3}{2} C \phi_{c}^{2} \eta_{z}^{2}\right)\right| 0\right\rangle \\
& =\hbar[\underbrace{A \int d^{2} z \Delta_{z x} \square \Delta_{y z}}_{(1.1)}-\underbrace{\left(B+3 C \phi_{c}^{2}\right) \int d^{2} z \Delta_{z x} \Delta_{y z}}_{(1.2)}-\underbrace{3 \lambda \int d^{2} z \Delta_{z x} \Delta_{y z} \Delta_{z z}}_{(1.3)}]
\end{aligned}
$$

Passando os propagadores para o espaço dos momentos, teremos:

$$
\begin{aligned}
& \text { (1.1) }=\hbar A \int d^{2} z \Delta_{z x} \square \Delta_{y z} \\
& =\hbar A \int d^{2} z \int \frac{d^{2} p}{(2 \pi)^{2}} \frac{1}{p^{2}+2 m^{2}} e^{i p(z-x)} \square \int \frac{d^{2} q}{(2 \pi)^{2}} \frac{1}{q^{2}+2 m^{2}} e^{i q(y-z)} \\
& =\hbar A \int \frac{d^{2} p}{(2 \pi)^{2}} \frac{d^{2} q}{(2 \pi)^{2}}(2 \pi)^{2} \delta^{(2)}(p-q)\left(-q^{2}\right) e^{-i(p x-q y)} \Delta(p) \Delta(q) \\
& =\int \frac{d^{2} p}{(2 \pi)^{2}} e^{-i p(x-y)} \Delta(p)\left[-\hbar A p^{2}\right] \Delta(p) \\
& (1.2)=-\hbar\left(B+3 C \phi_{c}^{2}\right) \int d^{2} z \Delta_{z x} \Delta_{y z} \\
& =-\hbar\left(B+3 C \phi_{c}^{2}\right) \int d^{2} z \frac{d^{2} p}{(2 \pi)^{2}} \Delta(p) e^{i p(z-x)} \frac{d^{2} q}{(2 \pi)^{2}} \Delta(q) e^{i q(y-z)} \\
& =-\hbar\left(B+3 C \phi_{c}^{2}\right) \int \frac{d^{2} p}{(2 \pi)^{2}} d^{2} q \delta^{(2)}(p-q) \Delta(p) \Delta(q) e^{-i p x+i q y} \\
& =\int \frac{d^{2} p}{(2 \pi)^{2}} e^{-i p(x-y)} \Delta(p)\left[-\hbar\left(B+3 C \phi_{c}^{2}\right)\right] \Delta(p) \\
& \text { (1.3) }=-3 \lambda \hbar \int d^{2} z \Delta_{z x} \Delta_{y z} \Delta_{z z} \\
& =-3 \lambda \hbar \int d^{2} z \frac{d^{2} p}{(2 \pi)^{2}} \frac{d^{2} q}{(2 \pi)^{2}} \frac{d^{2} k}{(2 \pi)^{2}} e^{i z(p-q)} e^{-i(p x-q y)} \Delta(p) \Delta(q) \Delta(k) \\
& =-3 \lambda \hbar \int \frac{d^{2} p}{(2 \pi)^{2}} \frac{d^{2} k}{(2 \pi)^{2}} e^{-i p(x-y)} \Delta(p) \Delta(p) \Delta(k) \\
& =\int \frac{d^{2} p}{(2 \pi)^{2}} e^{-i p(x-y)} \Delta(p)\left[-\hbar 3 \lambda \int \frac{d^{2} k}{(2 \pi)^{2}} \frac{1}{k^{2}+2 m^{2}}\right] \Delta(p)
\end{aligned}
$$


Calculamos, assim, os gráficos conexos 1PI do termo de primeira ordem da função de dois pontos. Agora vamos trabalhar com o termo de segunda ordem, (2), de F.10. Para obter uma aproximação $\hbar$, neste caso, devemos considerar os termos de $\mathcal{L}_{\text {int }}$ proporcionais a $\hbar^{3 / 2}$. De F.1, temos:

$$
\mathcal{L}_{\text {int }}=\hbar^{3 / 2}\left(-A \phi_{c} \partial_{\mu}^{2} \Phi+B \phi_{c} \Phi+C \phi_{c}^{3} \Phi+\lambda \phi_{c} \Phi^{3}\right)
$$

Substituindo no termo de segunda ordem, (2), de F.10:

$$
\begin{aligned}
(2)=\frac{1}{2 \hbar^{2}} \int d^{2} z_{1} d^{2} z_{2}\left\langle 0\left|T \eta_{x} \eta_{y} \mathcal{L}_{\text {int }}\left(z_{1}\right) \mathcal{L}_{\text {int }}\left(z_{2}\right)\right| 0\right\rangle \\
=\frac{1}{2 \hbar^{2}} \int d^{2} z_{1} d^{2} z_{2}\langle 0| T \eta_{x} \eta_{y}\left[\hbar^{3 / 2}\left(-A \phi_{c} \square \eta_{z 1}+B \phi_{c} \eta_{z 1}+C \phi_{c}^{3} \eta_{z 1}+\lambda \phi_{c} \eta_{z 1}^{3}\right)\right] \\
\quad \times\left[\hbar^{3 / 2}\left(-A \phi_{c} \square \eta_{z 2}+B \phi_{c} \eta_{z 2}+C \phi_{c}^{3} \eta_{z 2}+\lambda \phi_{c} \eta_{z 2}^{3}\right)\right]|0\rangle
\end{aligned}
$$

O único termo que fornece um gráfico conexo 1PI é dado por:

$$
\begin{aligned}
(2.1) & =\hbar \int d^{2} z_{1} d^{2} z_{2}\left\langle 0\left|T \eta_{x} \eta_{y}\left(\lambda \phi_{c} \eta_{z 1}^{3}\right)\left(\lambda \phi_{c} \eta_{z 2}^{3}\right)\right| 0\right\rangle \\
& =\hbar 18 \lambda^{2} \phi_{c}^{2} \int d^{2} z_{1} d^{2} z_{2} \Delta_{z_{1} x} \Delta_{z_{2} z_{1}} \Delta_{z_{1} z_{2}} \Delta_{y z_{2}}
\end{aligned}
$$

Passando os propagadores de (2.1) para o espaço dos momentos, tem-se: 


$$
\begin{aligned}
(2.1)= & \hbar 18 \lambda^{2} \phi_{c}^{2} \int d^{2} z_{1} d^{2} z_{2} \frac{d^{2} p}{(2 \pi)^{2}} e^{i p\left(z_{1}-x\right)} \Delta(p) \frac{d^{2} k_{1}}{(2 \pi)^{2}} e^{i k_{1}\left(z_{2}-z_{1}\right)} \Delta\left(k_{1}\right) \times \\
& \frac{d^{2} k_{2}}{(2 \pi)^{2}} e^{i k_{2}\left(z_{1}-z_{2}\right)} \Delta\left(k_{2}\right) \frac{d^{2} q}{(2 \pi)^{2}} e^{i q\left(y-z_{2}\right)} \Delta(q) \\
= & \hbar 18 \lambda^{2} \phi_{c}^{2} \int \frac{d^{2} p}{(2 \pi)^{2}} \frac{d^{2} q}{(2 \pi)^{2}} d^{2} k_{1} d^{2} k_{2} e^{i(p x+q y)} \Delta(p) \Delta(q) \Delta\left(k_{1}\right) \Delta\left(k_{2}\right) \times \\
= & \int \frac{d^{2} p}{(2 \pi)^{2}} e^{-i p(x-y)} \Delta(p)\left[\hbar 18 \lambda^{2} \phi_{c}^{2} \int \frac{d^{2} k}{(2 \pi)^{2}} \Delta(k) \Delta(p+k)\right] \Delta(p) \\
= & \int \frac{d^{2} p}{(2 \pi)^{2}} e^{-i p(x-y)} \Delta(p)\left[\hbar 18 \lambda^{2} \phi_{c}^{2} \int \frac{d^{2} k}{(2 \pi)^{2}} \frac{1}{\left[(p+k)^{2}+2 m^{2}\right]} \frac{1}{\left(k^{2}+2 m^{2}\right)}\right] \Delta(p)
\end{aligned}
$$

Juntando os termos de primeira e segunda ordem da função de dois pontos, os gráficos 1PI que contribuem para $\Gamma_{x}^{(2)}$ são dados por:

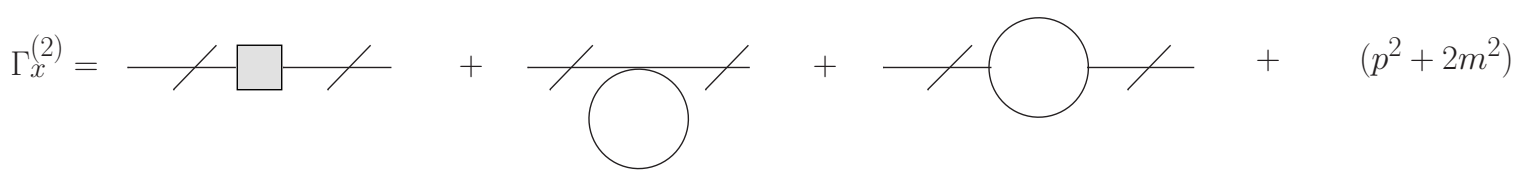

Sendo $\Gamma_{x}^{(2)}($ para $\hbar=1)$ igual a:

$$
\begin{aligned}
\Gamma_{x}^{(2)}=[- & \left(A p^{2}+B+3 C \frac{m^{2}}{\lambda}\right)-3 \lambda \int \frac{d^{2} k}{(2 \pi)^{2}} \frac{1}{k^{2}+2 m^{2}}+ \\
& \left.18 m^{2} \lambda \int \frac{d^{2} k}{(2 \pi)^{2}} \frac{1}{(p+k)^{2}+2 m^{2}} \frac{1}{k^{2}+2 m^{2}}\right]+\left(p^{2}+2 m^{2}\right)
\end{aligned}
$$

Antes de impor as condições de renormalização a $\Gamma_{x}^{(1)}$ e $\Gamma_{x}^{(2)}$, vamos simplificar a integral contida no termo F.19. 
Temos uma integral da forma:

$$
B=\int \frac{d^{2} k}{(2 \pi)^{2}} \frac{1}{(p+k)^{2}+m^{2}} \frac{1}{k^{2}+m^{2}}
$$

Para simplificar a notação, vamos reescrevê-la como:

$$
B=\underbrace{\int_{k} \frac{1}{(p+k)^{2}+m^{2}}}_{B_{1}} \underbrace{\int_{k} \frac{1}{k^{2}+m^{2}}}_{B_{2}}
$$

Trabalhando com $B_{1}$, tem-se:

$$
B_{1}=\int_{k} \frac{1}{k^{2}+p^{2}+2 k \cdot p+m^{2}}=\int_{k} \frac{1}{A+B}
$$

onde $A=k^{2}+m^{2}$ e $B=2 k \cdot p+p^{2}$.

Temos que ${ }^{2}$ :

$$
\frac{1}{A+B}=\frac{1}{A} \frac{1}{1+\frac{B}{A}}=\frac{1}{A}\left(1-\frac{B}{A}+\frac{B^{2}}{A^{2}}+\cdots\right)=\frac{1}{A}-\frac{B}{A^{2}}+\frac{B^{2}}{A^{3}}+\cdots
$$

Então:

$$
\begin{aligned}
B_{1}=\int_{k} \frac{1}{A+B} & =\int_{k}\left(\frac{1}{k^{2}+m^{2}}-\frac{p^{2}}{\left(k^{2}+m^{2}\right)^{2}}-\frac{2 k^{\mu} p_{\mu}}{\left(k^{2}+m^{2}\right)^{2}}+\frac{4 k^{\mu} p_{\mu} k^{\nu} p_{\nu}}{\left(k^{2}+m^{2}\right)^{3}}\right)+\cdots \\
& =\int_{k}\left(\frac{1}{k^{2}+m^{2}}-\frac{p^{2}}{\left(k^{2}+m^{2}\right)^{2}}+\frac{4 k^{\mu} p_{\mu} k^{\nu} p_{\nu}}{\left(k^{2}+m^{2}\right)^{3}}\right)+\cdots
\end{aligned}
$$

Mas

$$
\int \frac{k_{\mu} k_{\nu}}{A^{3}}=\delta^{\mu \nu} F
$$

onde F é uma constante. Além disso:

\footnotetext{
${ }^{2}$ Aqui usamos a expansão $\frac{1}{1+x}=1-x+x^{2}-x^{3}$
} 


$$
(d+1) F=\int_{k} \frac{k^{\mu} k^{\mu}+m^{2}-m^{2}}{A^{3}}=\int_{k} \frac{1}{A^{2}}-\frac{m^{2}}{A^{3}}
$$

sendo $d$ a dimensão do espaço. Então, para o caso $d=1$ :

$$
F=\frac{1}{2} \int_{k} \frac{1}{A^{2}}-\frac{1}{2} \int_{k} \frac{m^{2}}{A^{3}}
$$

Substituindo esta expressão em F.25, tem-se ${ }^{3}$ :

$$
\begin{aligned}
B_{1} & =\int_{k}\left(\frac{1}{k^{2}+m^{2}}+\int_{k} \frac{p^{2}}{\left(k^{2}+m^{2}\right)^{2}}-2 m^{2} p^{2} \int_{k} \frac{1}{\left(k^{2}+m^{2}\right)^{3}}\right)+\cdots \\
& =I_{1}\left(m^{2}\right)+p^{2} I_{2}\left(m^{2}\right)-2 m^{2} p^{2} I_{3}\left(m^{2}\right)+\cdots
\end{aligned}
$$

Temos então que F.21 é dada por:

$$
\begin{aligned}
B & =\int_{k} \frac{1}{(p+k)^{2}+m^{2}} \int_{k} \frac{1}{k^{2}+m^{2}}=\left(I_{1}\left(m^{2}\right)+p^{2} I_{2}\left(m^{2}\right)-2 m^{2} p^{2} I_{3}\left(m^{2}\right)\right) I_{1}\left(m^{2}\right) \\
& =I_{2}\left(m^{2}\right)+p^{2} I_{3}\left(m^{2}\right)-2 m^{2} p^{2} I_{4}\left(m^{2}\right)
\end{aligned}
$$

Ou seja, conseguimos expandir a integral da forma F.21 em potências de $p^{2}$, o que facilitará muito os cálculos ao aplicar as condições de renormalização.

Reescrevendo os resultados obtidos para $\Gamma^{(1)}$ e $\Gamma^{(2)}$, usando esta expansão em potências de $p^{2}$, teremos:

$$
\Gamma_{x}^{(1)}=-\frac{m}{\sqrt{\lambda}}\left[3 \lambda I_{1}\left(2 m^{2}\right)+B+\frac{m^{2}}{\lambda} C\right]
$$

\footnotetext{
${ }^{3}$ Aqui começaremos a utilizar a notação $I_{n}$ para representar as integrais, em que $I_{n}\left(m^{2}\right)=$ $\int \frac{1}{\left(k^{2}+m^{2}\right)^{n}}$
} 


$$
\begin{aligned}
\Gamma_{x}^{(2)}= & -3 \lambda I_{1}\left(2 m^{2}\right)+18 \lambda m^{2}\left[I_{2}\left(2 m^{2}\right)+p^{2} I_{3}\left(2 m^{2}\right)-4 m^{2} p^{2} I_{4}\left(2 m^{2}\right)\right] \\
& -\left(A p^{2}+B+3 C \frac{m^{2}}{\lambda}\right)+\left(p^{2}+2 m^{2}\right)
\end{aligned}
$$

Impondo as condições de renormalização:

$$
\left\{\begin{array}{l}
\Gamma^{(1)}=0 \\
\Gamma^{(2)}\left(p^{2}=0\right)=2 m^{2} \\
\left.\frac{d \Gamma^{(2)}}{d p^{2}}\right|_{\left(p^{2}=0\right)}=0
\end{array}\right.
$$

obtem-se os seguintes valores para os contratermos $A, B$ e $C$ :

$$
\begin{gathered}
A=18 \lambda m^{2} \int \frac{d^{2} k}{(2 \pi)^{2}} \frac{1}{\left(k^{2}+2 m^{2}\right)^{3}}-72 \lambda m^{4} \int \frac{d^{2} k}{(2 \pi)^{2}} \frac{1}{\left(k^{2}+2 m^{2}\right)^{4}} \\
B=-3 \lambda \int \frac{d^{2} k}{(2 \pi)^{2}} \frac{1}{\left(k^{2}+2 m^{2}\right)}-9 \lambda m^{2} \int \frac{d^{2} k}{(2 \pi)^{2}} \frac{1}{\left(k^{2}+2 m^{2}\right)^{2}} \\
C=9 \lambda^{2} \int \frac{d^{2} k}{(2 \pi)^{2}} \frac{1}{\left(k^{2}+2 m^{2}\right)^{2}}
\end{gathered}
$$

\section{F.2. Campo bosônico acoplado a campo fermiônico}

Conforme foi visto na seção 5.4, a lagrangiana renormalizada do modelo $\lambda \phi^{4}$ acoplado a um campo fermiônico, após expansão do campo em $\phi=\phi_{c}+\Phi$, é dada por:

$$
\begin{aligned}
\mathcal{L}_{R} & =\hbar^{0}\left(-\frac{1}{2} \phi_{c} \partial_{\mu}^{2} \phi_{c}-\frac{1}{2} m^{2} \phi^{2}+\frac{1}{4} \lambda \phi_{c}^{4}+\frac{m^{4}}{4 \lambda}\right) \\
& +\hbar^{1 / 2}\left(-\partial_{\mu}^{2} \phi_{c}-m^{2} \phi_{c}+\lambda \phi_{c}^{3}\right) \Phi \\
& +\hbar\left(-\frac{1}{2} \Phi \partial_{\mu}^{2} \Phi-\frac{1}{2} m^{2} \Phi^{2}+\frac{3}{2} \lambda \phi_{c}^{2} \Phi^{2}-\frac{A}{2} \phi_{c} \partial_{\mu}^{2} \phi_{c}+\frac{B}{2} \phi_{c}^{2}+\frac{C}{4} \phi_{c}^{4}+H-\bar{\Psi} \not \partial \Psi-i g \bar{\Psi} \Psi \phi_{c}\right) \\
& +\hbar^{3 / 2}\left(\lambda \phi_{c} \Phi^{3}-A \phi_{c} \partial_{\mu}^{2} \Phi+B \phi_{c} \Phi+C \phi_{c}^{3} \Phi-i g \bar{\Psi} \Psi \Phi\right) \\
& +\hbar^{2}\left(\frac{\lambda}{4} \Phi^{4}-\frac{A}{2} \Phi \partial_{\mu}^{2} \Phi+\frac{B}{2} \Phi^{2}+\frac{3}{2} C \phi_{c}^{2} \Phi^{2}-F \bar{\Psi} \not \partial \Psi-i G \bar{\Psi} \Psi \phi_{c}\right)
\end{aligned}
$$


O propagador do férmion é dado por E.29:

$$
S_{F}(p)=-i \frac{\gamma^{\nu} p_{\nu}+M}{p^{2}+M^{2}}
$$

Analogamente ao que foi feito para o caso do campo puramente bosônico, vamos determinar a primeira ordem da função de 1 ponto (Veja F.3), na aproximação de $\hbar^{1 / 2}$, ou seja, utilizando termos de $\mathcal{L}_{\text {int }}$ proporcionais a $\hbar^{3 / 2}$. De F.37, temos:

$$
\mathcal{L}_{\text {int }}=\hbar^{3 / 2}\left(\lambda \phi_{c} \Phi^{3}-A \phi_{c} \partial_{\mu}^{2} \Phi+B \phi_{c} \Phi+C \phi_{c}^{3} \Phi-i g \bar{\Psi} \Psi \Phi\right)
$$

Substituindo no termo de primeira ordem, (1), da função de 1 ponto, tem-se:

$$
\begin{aligned}
(1) & =\left\langle 0\left|T \eta_{x}\left(-\frac{1}{\hbar}\right) \int \mathcal{L}_{i n t} d^{2} z\right| 0\right\rangle \\
& =\underbrace{\left\langle 0\left|T \eta_{x}\left(\frac{-1}{\hbar}\right) \int d^{2} z\left(-A \phi_{c} \square \eta_{z}+B \phi_{c} \eta_{z}+C \phi_{c}^{3} \eta_{z}+\lambda \phi_{c} \eta_{z}^{3}\right) \hbar^{3 / 2}\right| 0\right\rangle}_{B} \\
& +\underbrace{\left\langle 0\left|T \eta_{x}\left(-\frac{1}{\hbar}\right) \int d^{2} z\left(-i g \bar{\Psi}_{z} \Psi_{z} \eta_{z}\right) \hbar^{3 / 2}\right| 0\right\rangle}_{F 1} .
\end{aligned}
$$

Os gráficos conexos 1PI, pertencentes à parte puramente bosônica B já foram cal- 
culados na seção anterior. Vamos proceder à contribuição fermiônica $F 1$. Então ${ }^{4}$ :

$$
\begin{aligned}
(F 1) & =\left\langle 0\left|T \eta_{x}\left(-\frac{1}{\hbar}\right) \int d^{2} z\left(-i g \bar{\Psi}_{z} \Psi_{z} \eta_{z}\right) \hbar^{3 / 2}\right| 0\right\rangle \\
& =\hbar^{1 / 2} i g \int d^{2} z \Delta_{z x} S_{z z}=\int d^{2} z \Delta_{z x}\left[\hbar^{1 / 2} i g \int \frac{d^{2} p}{(2 \pi)^{2}} \operatorname{Tr} S(p)\right] \\
& =\int d^{2} z \Delta_{z x}\left[\hbar^{1 / 2} i g \int \frac{d^{2} p}{(2 \pi)^{2}} \operatorname{Tr}\left(i \frac{\gamma^{\nu} p_{\nu}-M}{p^{2}+M^{2}}\right)\right] \\
& =\int d^{2} z \Delta_{z x}\left[\hbar^{1 / 2} 2 g^{2} \frac{m}{\sqrt{\lambda}} \int \frac{d^{2} p}{(2 \pi)^{2}} \frac{1}{p^{2}+M^{2}}\right] .
\end{aligned}
$$

Assim, os gráficos 1PI que contribuem para $\Gamma_{x}^{(1)}$, contando as contribuições bosônica e fermiônica, são dados por:

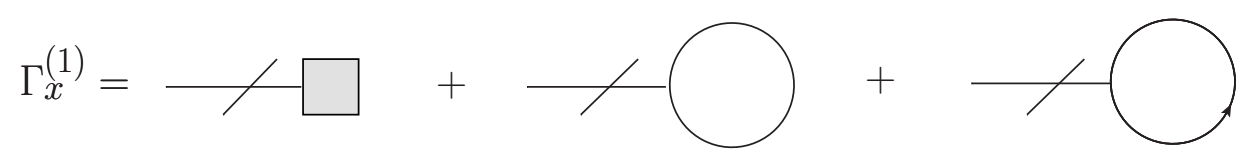

Sendo $\Gamma_{x}^{(1)}($ para $\hbar=1)$ :

$$
\Gamma_{x}^{(1)}=-\frac{m}{\sqrt{\lambda}}\left[3 \lambda \int \frac{d^{2} p}{(2 \pi)^{2}} \frac{1}{\left(p^{2}+2 m^{2}\right)}+B+\frac{m^{2}}{\lambda} C-2 g^{2} \int \frac{d^{2} p}{(2 \pi)^{2}} \frac{1}{p^{2}+M^{2}}\right]
$$

Agora vamos trabalhar com o termo de segunda ordem da função de 2 pontos (Veja F.10), pois nem os termos de segunda ordem da função de 1 ponto e nem os de primeira ordem da função de 2 pontos, fornecem gráficos conexos. Como queremos a aproximação da ordem de $\hbar$, novamente precisamos de termos de $\mathcal{L}_{\text {int }}$ proporcionais a $\hbar^{3 / 2}$. Então:

$$
\mathcal{L}_{\text {int }}=\hbar^{3 / 2}\left(\lambda \phi_{c} \Phi^{3}-A \phi_{c} \partial_{\mu}^{2} \Phi+B \phi_{c} \Phi+C \phi_{c}^{3} \Phi-i g \bar{\Psi} \Psi \Phi\right)
$$

Substituindo no termo de segunda ordem de F.10:

\footnotetext{
${ }^{4}$ Observe que $\operatorname{Tr}$ refere-se à operação de traço.
} 


$$
\begin{aligned}
(2) & =\frac{1}{2 \hbar^{2}} \int d^{2} z_{1} d^{2} z_{2}\langle 0| T \eta_{x} \eta_{y}\left[\hbar^{3 / 2}\left(\lambda \phi_{c} \eta_{z 1}^{3}-A \phi_{c} \partial_{\mu}^{2} \eta_{z 1}+B \phi_{c} \eta_{z 1}+C \phi_{c}^{3} \eta_{z 1}\right)\right] \\
& {\left[\hbar^{3 / 2}\left(\lambda \phi_{c} \eta_{z 2}^{3}-A \phi_{c} \partial_{\mu}^{2} \eta_{z 2}+B \phi_{c} \eta_{z 2}+C \phi_{c}^{3} \eta_{z 2}\right)\right]|0\rangle } \\
+ & \underbrace{\frac{1}{2 \hbar^{2}} \int d^{2} z_{1} d^{2} z_{2}\left\langle 0\left|T \eta_{x} \eta_{y}\left[\hbar^{3 / 2}\left(-i g \bar{\Psi}_{z 1} \Psi_{z 1} \eta_{z 1}\right)\right]\left[\hbar^{3 / 2}\left(-i g \bar{\Psi}_{z 2} \Psi_{z 2} \eta_{z 2}\right)\right]\right| 0\right\rangle}_{(F 2)}
\end{aligned}
$$

O termo (F2) é a contribuição fermiônica, os demais são a contribuição bosônica já calculada anteriormente. Temos:

$$
\begin{aligned}
& (F 2)=\frac{\hbar}{2} \int d^{2} z_{1} d^{2} z_{2}\left\langle 0\left|T \eta_{x} \eta_{y}\left(-i g \bar{\Psi}_{z 1} \Psi_{z 1} \eta_{z 1}\right)\left(-i g \bar{\Psi}_{z 2} \Psi_{z 2} \eta_{z 2}\right)\right| 0\right\rangle \\
& =-\hbar g^{2} \int d^{2} z_{1} d^{2} z_{2} \Delta_{z_{1} x} \Delta_{y z_{2}} S_{z_{2} z_{1}} S_{z_{1} z_{2}} \\
& =-\hbar g^{2} \int d^{2} z_{1} d^{2} z_{2}\left[\frac{d^{2} p}{(2 \pi)^{2}} \Delta(p) e^{i p\left(z_{1}-x\right)}\right]\left[\frac{d^{2} k_{1}}{(2 \pi)^{2}} S\left(k_{1}\right) e^{i k_{1}\left(z_{2}-z_{1}\right)}\right] \times \\
& {\left[\frac{d^{2} k_{2}}{(2 \pi)^{2}} S\left(k_{2}\right) e^{i k_{2}\left(z_{1}-z_{2}\right)}\right]\left[\frac{d^{2} q}{(2 \pi)^{2}} \Delta(q) e^{i q\left(y-z_{2}\right)}\right]} \\
& =-\hbar g^{2} \int \frac{d^{2} p}{(2 \pi)^{2}} \frac{d^{2} q}{(2 \pi)^{2}} d^{2} k_{1} d^{2} k_{2} e^{-i(p x-q y)} \Delta(p) S\left(k_{1}\right) S\left(k_{2}\right) \Delta(q) \times \\
& \delta^{(2)}\left(k_{1}-k_{2}-p\right) \delta^{(2)}\left(k_{2}-k_{1}+q\right) \\
& =\int \frac{d^{2} p}{(2 \pi)^{2}} e^{-i p(x-y)} \Delta(p) \underbrace{\left[\hbar g^{2} \int \frac{d^{2} k}{(2 \pi)^{2}} S(k) S(p+k)\right]}_{G_{2}} \Delta(p)
\end{aligned}
$$

Onde 


$$
\begin{aligned}
G_{2} & =\hbar g^{2} \int \frac{d^{2} k}{(2 \pi)^{2}} S_{\alpha \alpha}(k) S_{\alpha \alpha}(p+k)=\hbar g^{2} \int \frac{d^{2} k}{(2 \pi)^{2}} i \frac{(\not k-M)_{\alpha \alpha}}{\left(k^{2}+M^{2}\right)} i \frac{(\not p-\not k-M)_{\alpha \alpha}}{\left[(p+k)^{2}+M^{2}\right]} \\
& =-\hbar g^{2} \int \frac{d^{2} k}{(2 \pi)^{2}} \frac{\left(\not k p p-\not k \not k-\not k M-\not p M+\not k M+M^{2}\right)_{\alpha \alpha}}{\left(k^{2}+M^{2}\right)\left[(p+k)^{2}+M^{2}\right]} \\
& =-\hbar g^{2} \int \frac{d^{2} k}{(2 \pi)^{2}} \frac{\left(-\delta^{\mu \nu} k_{\mu} p_{\nu}-\delta^{\mu \nu} k_{\mu} k_{\nu}+M^{2}\right)_{\alpha \alpha}}{\left(k^{2}+M^{2}\right)\left[(p-k)^{2}+M^{2}\right]} \\
& =\hbar 2 g^{2} \int \frac{d^{2} k}{(2 \pi)^{2}} \frac{\left(p \cdot k+k^{2}-M^{2}\right)}{\left(k^{2}+M^{2}\right)\left[(p+k)^{2}+M^{2}\right]}
\end{aligned}
$$

Finalmente, juntando a contribuição bosônica e fermiônica, os gráficos 1PI que contribuem para $\Gamma_{x}^{(2)}$ são dados por:

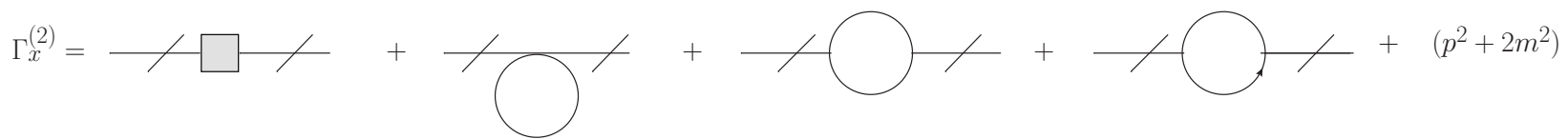

Sendo $\Gamma_{x}^{(2)}($ para $\hbar=1)$ igual a:

$$
\begin{aligned}
\Gamma_{x}^{(2)}=[ & -3 \lambda \int \frac{d^{2} k}{(2 \pi)^{2}} \frac{1}{p^{2}+2 m^{2}}+18 m^{2} \lambda \int \frac{d^{2} k}{(2 \pi)^{2}} \frac{1}{\left[(p+k)^{2}+2 m^{2}\right]} \frac{1}{\left(k^{2}+2 m^{2}\right)}+ \\
& \left.-\left(A p^{2}+B+3 \frac{m^{2}}{\lambda} C\right)+2 g^{2} \int \frac{d^{2} k}{(2 \pi)^{2}} \frac{\left(p \cdot k+k^{2}-M^{2}\right)}{\left(k^{2}+M^{2}\right)\left[(p+k)^{2}+M^{2}\right]}\right]+\left(p^{2}+2 m^{2}\right)
\end{aligned}
$$

Antes de aplicar as condições de renormalização, vamos simplificar a integral que aparece em F.46. Temos: 


$$
\begin{aligned}
I & =\int \frac{d^{2} k}{(2 \pi)^{2}} \frac{\left(p . k+k^{2}-M^{2}\right)}{\left(k^{2}+M^{2}\right)\left[(p+k)^{2}+M^{2}\right]}=\int_{k} \frac{1}{2}\left(\left(k^{2}+M^{2}\right)+\left((k+p)^{2}+M^{2}\right)-p^{2}-4 M^{2}\right) \\
& =\underbrace{\int_{k} \frac{1}{2}\left[\frac{1}{(k+p)^{2}+M^{2}}+\frac{1}{k^{2}+M^{2}}\right]}_{F_{1}}-\underbrace{\frac{p^{2}+4 M^{2}}{2} \int_{k} \frac{1}{\left(k^{2}+M^{2}\right)\left[(p+k)^{2}+M^{2}\right]}}_{F_{2}}
\end{aligned}
$$

Utilizando o resultado de F.30 (assim como a notação), é fácil ver que:

$$
\begin{gathered}
F_{1}=I_{1}\left(M^{2}\right)+\frac{p^{2}}{2} I_{2}\left(M^{2}\right)-p^{2} M^{2} I_{3}\left(M^{2}\right) \\
F_{2}=\frac{p^{2}}{2} I_{2}\left(M^{2}\right)-2 M^{2} I_{2}\left(M^{2}\right)-2 M^{2} p^{2} I_{3}(M 2)+4 M^{4} p^{2} I_{4}\left(M^{2}\right)+O\left(p^{4}\right) \\
I=F_{1}+F_{2}=I_{1}\left(M^{2}\right)-2 M^{2} I_{2}\left(M^{2}\right)-3 M^{2} p^{2} I_{3}\left(M^{2}\right)+4 M^{4} p^{2} I_{4}\left(M^{2}\right)
\end{gathered}
$$

Vamos reescrever $\Gamma^{(1)}$ e $\Gamma^{(2)}$ utilizando esta expansão e a notação de $I_{n}$ :

$$
\begin{gathered}
\Gamma_{x}^{(1)}=-\frac{m}{\sqrt{\lambda}}\left[3 \lambda I_{1}\left(2 m^{2}\right)+B+\frac{m^{2}}{\lambda} C-2 g^{2} I_{1}\left(M^{2}\right)\right] \\
\Gamma_{x}^{(2)}=\left(p^{2}+2 m^{2}\right)-3 \lambda I_{1}\left(2 m^{2}\right)+18 \lambda m^{2}\left[I_{2}\left(2 m^{2}\right)+p^{2} I_{3}\left(2 m^{2}\right)-4 m^{2} p^{2} I_{4}\left(2 m^{2}\right)\right] \\
-\left(A p^{2}+B+3 C \frac{m^{2}}{\lambda}\right)+2 g^{2}\left[I_{1}\left(M^{2}\right)-2 M^{2} I_{2}(M 2)-3 M p^{2} I_{3}\left(M^{2}\right)+4 M^{4} p^{2} I_{4}\left(M^{2}\right)\right]
\end{gathered}
$$

Impondo as condições de renormalização F.33, obtem-se para os contratermos:

$$
\begin{aligned}
A= & -72 \lambda m^{4} \int \frac{d^{2} k}{(2 \pi)^{2}} \frac{1}{\left(k^{2}+2 m^{2}\right)^{4}}+18 \lambda m^{2} \int \frac{d^{2} k}{(2 \pi)^{2}} \frac{1}{\left(k^{2}+2 m^{2}\right)^{3}}+ \\
& -6 g^{2} M^{2} \int \frac{d^{2} k}{(2 \pi)^{2}} \frac{1}{\left(k^{2}+M^{2}\right)^{3}}+8 g^{2} M^{4} \int \frac{d^{2} k}{(2 \pi)^{2}} \frac{1}{\left(k^{2}+M^{2}\right)^{4}}
\end{aligned}
$$




$$
\begin{aligned}
B= & -3 \lambda \int \frac{d^{2} k}{(2 \pi)^{2}} \frac{1}{\left(k^{2}+2 m^{2}\right)}-9 \lambda m^{2} \int \frac{d^{2} k}{(2 \pi)^{2}} \frac{1}{\left(k^{2}+2 m^{2}\right)^{2}}+ \\
& +2 g^{2} \int \frac{d^{2} k}{(2 \pi)^{2}} \frac{1}{\left(k^{2}+M^{2}\right)}+2 g^{2} M^{2} \int \frac{d^{2} k}{(2 \pi)^{2}} \frac{1}{\left(k^{2}+M^{2}\right)^{2}} \\
& C=9 \lambda^{2} \int \frac{d^{2} k}{(2 \pi)^{2}} \frac{1}{\left(k^{2}+2 m^{2}\right)^{2}}-2 g^{4} \int \frac{d^{2} k}{(2 \pi)^{2}} \frac{1}{\left(k^{2}+M^{2}\right)^{2}}
\end{aligned}
$$





\section{Referências bibliográficas}

[1] Kaku, Michio. Quantum Field Theory. Oxford (1993).

[2] Rajaraman, R. Solitons and Instantons. North Holland (1982).

[3] Ryder, L. H. Quantum Field Theory. Cambridge University Press, 1996 (Second Edition).

[4] Vachaspati, Tanmay. Kinks and Domain Walls. Cambridge (2006).

[5] R. Dashen, B. Hasslacher e A. Neveau, Phys. Rev. D10 (1974) 4114; 4130.

[6] Das, Ashok. Finite Temperature Field Theory. World Scientific (1997).

[7] D. Bazeia, O.J.P. Éboli, G.C. Marques, A.J.Silva. Z.Phys.C 46, 457 (1990).

[8] Lemos, Nivaldo. Mecânica Analítica. Editora Livraria da Física, São Paulo (2004).

[9] Gottfried, Kurt. Yan, Tung-Mow. Quantum Mechanics: Fundamentals. Springer (2004).

[10] Greiner, W. Reinhardt, J. Field Quantization. Springer-Verlag, Berlin (1996).

[11] Salinas, S.R.A. Introdução à Física Estatística. Edusp, São Paulo (2005).

[12] Peskin, Michael E. Schroeder, Daniel V. An Introduction to Quantum Field Theory. Addison-Wesley (1995). 
[13] Kapusta, Joseph I. Finite-Temperature Field Theory. Cambridge University Press (1989).

[14] Kadanoff, Leo P. Baym, Gordon. Quantum Statistical Mechanics. W.A.Benjamin, Inc. New York (1962).

[15] Ramond, Pierre. Field Theory: A modern Primer. Addison-Wesley (1989).

[16] Itzykson, Claude. Zuber, Jean-Bernard. Quantum Field Theory. McGraw-Hill (1980).

[17] Flugge, S. Practical Quantum Mechanics. Springer-Verlag, Berlin (1999).

[18] Hatfield, B. Quantum Field Theory of Point Particles and Strings. AddisonWesley, Redwood City (1989).

[19] Maggiore, Michele. A modern Introduction to Quantum Field Theory. Oxford University Press (2005).

[20] Huang, Kerson. Quantum Field Theory - From Operators to Path Integrals. John Wiley and Sons (1998). 